\title{
DISCRETE CONNECTIONS ON PRINCIPAL BUNDLES: THE DISCRETE ATIYAH SEQUENCE
}

\author{
JAVIER FERNÁNDEZ, MARIANA JUCHANI, AND MARCELA ZUCCALLI
}

\begin{abstract}
In this work we study discrete analogues of an exact sequence of vector bundles introduced by M. Atiyah in 1957, associated to any smooth principal $G$-bundle $\pi: Q \rightarrow Q / G$. In the original setting, the splittings of the exact sequence correspond to connections on the principal bundle $\pi$. The discrete analogues that we consider here can be studied in two different categories: the category of fiber bundles with a (chosen) section, Fbs, and the category of local Lie groupoids, $l L G p d$. In $F b s$ we find a correspondence between a) (semi-local) splittings of the discrete Atiyah sequence (DAS) of $\pi$, b) discrete connections on the same bundle $\pi$, and c) isomorphisms of the DAS with certain fiber product extensions in Fbs. We see that the right splittings of the DAS (in Fbs) are not necessarily right splittings in lLGpd: we use this obstruction to define the discrete curvature of a discrete connection. Then, there is a correspondence between the splittings of the DAS in $l L G p d$ and discrete connections with trivial discrete curvature. We also introduce a semidirect product between (some) local Lie groupoids and prove that there is a correspondence between semidirect product extensions and splittings of the DAS in $l L G p d$.
\end{abstract}

\section{INTRODUCTION}

Connections on bundles are useful tools in Geometry. They can be used to probe the intrinsic structures of the underlying bundle space; they can also be used to obtain convenient global expressions of local computations. Even in Physics, connections on vector and principal bundles are essential ingredients of modern Gauge Theory (see [DV80]). In the classical formulation, a connection on a principal $G$ bundle $\pi: Q \rightarrow Q / G$ can be defined by giving a smooth and regular distribution Hor $\subset T Q$ that is, at each $q \in Q$, complementary to the vertical distribution $\mathcal{V}=\operatorname{ker}(T \pi) \subset T Q$ and is $G$-equivariant for the lifted $G$-action on $T Q$. Alternatively, a connection is determined by a $\mathfrak{g}$-valued 1 -form on $T Q$, where $\mathfrak{g}$ is the Lie algebra of $G$, or by a horizontal lift $h: \pi^{*}(T(Q / G)) \rightarrow T Q$, in both cases satisfying appropriate conditions (see $[\mathrm{KN} 96]$ ).

Lagrangian Mechanical systems on a configuration manifold $Q$ are dynamical systems on the tangent bundle $T Q$, whose trajectories are critical points of a functional determined by a Lagrangian function $L: T Q \rightarrow \mathbb{R}$. Approximate trajectories of these systems can be obtained by appropriate discretizations of the problem: more precisely, a discrete-time system on $Q \times Q$ whose trajectories are the critical points of a functional determined by a discrete Lagrangian $L_{d}: Q \times Q \rightarrow \mathbb{R}$. It seems natural to view $Q \times Q$ as a discrete version of $T Q$; indeed, while elements $v_{q} \in T_{q} Q$ can be seen as velocity vectors at the point $q$, a discrete (time) version of this picture replaces $v_{q}$ with the pair $\left(q, q^{\prime}\right) \in Q \times Q$, seen as a short trajectory from $q$ to $q^{\prime}$ in the general direction of $v_{q}$ (see [MW01]). Following A. Weinstein's [Wei96], This idea has been expanded to deal with more general

2020 Mathematics Subject Classification. 53C05, 53C15, $70 \mathrm{G} 45$.

This research was partially supported by grants from the Universidad Nacional de Cuyo (grants 06/C567 and 06/C496), Universidad Nacional de La Plata, and CONICET. 
continuous dynamical systems, not necessarily defined on $T Q$ but, rather, on Lie algebroids and with discrete approximations given by dynamical systems defined on Lie groupoids (see [CdLM+06], [MMdDM15] and [MMdDS15]).

Just as for continuous mechanical systems connections $\mathcal{A}$ on principal bundles $\pi: Q \rightarrow Q / G$ are important tools in the study of symmetries, especially in the reduction and reconstruction processes, in the discrete-time realm it is interesting and useful to consider a decomposition of $Q \times Q$ into "vertical" and "horizontal" vectors (see, for instance, [FTZ10]). A notion of discrete connection on a principal bundle was proposed by M. Leok, J. Marsden and A. Weinstein in [LMW05] and [Leo04] and, later, refined by some of us in [FZ13]. Briefly, a discrete connection on $\pi$ is determined by a submanifold Hor $_{\mathcal{A}_{d}} \subset Q \times Q$ that is complementary to a discrete vertical manifold $\mathcal{V}_{d}$, all understood in the appropriate sense. Just as their continuous counterparts, discrete connections can be alternatively determined through discrete connection forms $\mathcal{A}_{d}: Q \times Q \rightarrow G$, or discrete horizontal lifts $h_{d}: Q \times(Q / G) \rightarrow Q \times Q$. Things are, indeed, a bit more subtle as there may be no discrete connections for nontrivial principal bundles: usually, for instance, the discrete connection form $\mathcal{A}_{d}$ is defined in an open subset $\mathfrak{U} \subset Q \times Q$ called its domain. Below, in Section 2.2, we give complete definitions and the reader should consult [FZ13] for further details.

Associated to any principal $G$-bundle $\pi: Q \rightarrow Q / G$, M. Atiyah introduced in [Ati57] the following short exact sequence of vector bundles over $Q / G$, known nowadays as the Atiyah sequence of $\pi$,

$$
0 \longrightarrow \widetilde{\mathfrak{g}} \stackrel{\phi_{1}}{\longrightarrow}(T Q) / G \stackrel{\phi_{2}}{\longrightarrow} T(Q / G) \longrightarrow 0
$$

where $\widetilde{\mathfrak{g}}=(Q \times \mathfrak{g}) / G$ (for $G$ acting on $\mathfrak{g}$, the Lie algebra of $G$, by the Adjoint action) is the adjoint bundle $e^{1}, \phi_{1}\left(\pi^{Q \times \mathfrak{g}, G}(q, \xi)\right)=\pi^{T Q, G}\left(\xi_{Q}(q)\right)$ and $\phi_{2}\left(\pi^{T Q, G}\left(v_{q}\right)\right)=$ $T_{q} \pi\left(v_{q}\right)$. He also proved that the splittings of the sequence correspond precisely to connections on $\pi$. It is also possible to view (1.1) as an exact sequence in the category of Lie algebroids, as presented in [Mac87]. In this case, a right splitting $s_{R}$ of (1.1) (in the category of vector bundles) may not be a morphism of Lie algebroids, with the obstruction being the curvature of the connection associated to $s_{R}$.

A discrete analogue of (1.1) is sketched in [LMW05], although the precise algebraic or categorical context is not made explicit, which is a nontrivial point because the categories possibly involved are not abelian, so that the basic notions, like exactness, require some careful interpretation. The main objective of this work is to provide a precise definition of a discrete Atiyah sequence and prove that results analogous to the ones described in the previous paragraph for the continuous setting are valid in the discrete setting. More precisely, for any principal $G$-bundle $\pi: Q \rightarrow Q / G$, if we denote the $G$ action on $Q$ by $l^{Q}$, we consider the sequence

$$
(Q \times G) / G \stackrel{F_{1}}{\longrightarrow}(Q \times Q) / G \stackrel{F_{2}}{\longrightarrow}(Q / G) \times(Q / G)
$$

for

(1.3)

$$
F_{1}\left(\pi^{Q \times G, G}(q, g)\right)=\pi^{Q \times Q, G}\left(q, l_{g}^{Q}(q)\right) \quad \text { and } \quad F_{2}\left(\pi^{Q \times Q, G}\left(q_{0}, q_{1}\right)\right)=\left(\pi\left(q_{0}\right), \pi\left(q_{1}\right)\right)
$$

as a sequence of smooth fiber bundles over $Q / G$. The space $\widetilde{G}=(Q \times G) / G$, obtained when $G$ acts on the principal bundle $Q$ in the natural way and on $G$ by conjugation, will be called the conjugate bundle over $Q / G$. In fact, for technical reasons we have to work in the category $F b s_{Q / G}$ of fiber bundles with a section

\footnotetext{
${ }^{1}$ When the Lie group $X$ acts on the manifold $X$, we denote the quotient map by $\pi^{X, G}: X \rightarrow$ $X / G$.
} 
(over $Q / G$ ). As a first step we prove that (1.2) is an extension (Definition 3.4) of $(Q / G) \times(Q / G)$ by $\widetilde{G}$ in the $F b s_{Q / G}$ category. Just as discrete connections may not be globally defined, splittings of (1.2) may not be defined globally but, rather, semilocally (Definition 3.8). Then we prove that the (semi-local) left splittings of (1.2) satisfying a certain equivariance condition are in a bijective correspondence with discrete connections on $\pi$, while (semi-local) right splittings of (1.2) are in a bijective correspondence with discrete horizontal lifts on $\pi$. As discrete horizontal lifts and discrete connections on $\pi$ are known to be equivalent notions, we have proved the equivalence between some left splittings of (1.2), right splittings of (1.2), and discrete connections on $\pi$. Of course, the correspondence between left and right splittings of an exact sequence is well known in other categories, but it is not at all automatic in this non-abelian category. Another standard result, for instance in the category of vector bundles, is that split exact sequences are equivalent to direct sum sequences. We prove that (1.2) is semi-locally right (or left) split if and only if the sequence is semi-locally equivalent to a certain fiber product extension of $(Q / G) \times(Q / G)$ by $\widetilde{G}$. A complete "map" of the relationship between the different objects considered so far is provided by Theorem 3.35 .

In the continuous case additional aspects of connections on $\pi$ are revealed when they are considered in the context of Lie algebroids: a connection $\mathcal{A}$ on $\pi$ defines the map $s_{R}$ in the category of vector bundles that is a morphism of Lie algebroids if and only if its curvature $\mathcal{B}$ vanishes. In the same spirit, a discrete connection $\mathcal{A}_{d}$ defines a map from $Q \times Q$ into $G$ and, as $Q \times Q$ as well as $G$ carry natural Lie groupoid structures, it is natural to ask if $\mathcal{A}_{d}$ is a morphism of Lie groupoids. It turns out that the interesting question is if it is a morphism from $Q \times Q$ into the Lie groupoid associated to the opposite Lie group $G^{o p}$. In general $\mathcal{A}_{d}$ need not be such a morphism and we use the obstruction to define the curvature $\mathcal{B}_{d}$ of $\mathcal{A}_{d}$ (Definition 4.6). Then, naturally, $\mathcal{A}_{d}$ determines a morphism of Lie groupoids if and only if $\mathcal{B}_{d}$ is trivial. Discrete connections with trivial curvature are called flat.

Just as in the continuous case where the sequence (1.1) can also be seen as a sequence in the category of Lie algebroids, it is noticed in [LMW05] that (1.2) can also be seen as a sequence in the category of Lie groupoids $L G p d$, a fact that we also review here, and that has been also considered elsewhere in the literature ([And04] and [CR18]). Our interest is to explore the relationship between the (right) splittings of (1.2) in the LGpd category and discrete connections on $\pi$. Still, a little twist is required because discrete connections are defined in open subsets $\mathfrak{U} \subset Q \times Q$, so that the right splittings that they induce are defined on open subsets of $(\pi \times \pi)(\mathfrak{U}) \subset(Q / G) \times(Q / G)$ and cannot be morphisms in $L G p d$, that are globally defined. So we move from the $L G p d$ category to the category of local Lie groupoids $l L G p d$. We prove that (1.2) is an extension in the $l L G p d$ category (Definition 5.41). Discrete connections on $\pi$ induce (semi-local) right splittings $s_{R}$ of (1.2) in the $F b s_{Q / G}$ category, but not necessarily in the $l L G p d_{Q / G}$ category; those $s_{R}$ that split the discrete Atiyah sequence in the $l L G p d_{Q / G}$ category are precisely those coming from flat discrete connections on $\pi$. In fact, we prove in Proposition 5.44 that there is a bijective correspondence between the right splittings of the discrete Atiyah sequence in the $l L G p d_{Q / G}$ category and the flat discrete connections on $\pi$.

Inspired by the structure introduced in [MM10], we consider the semidirect product of a (totally intransitive, see Definition 5.7) Lie groupoid by a local Lie groupoid, that is a local Lie groupoid and can be used to construct a certain semidirect product extension in $l L G p d$. Theorem 5.58 proves that, for a given extension in the $l L G p d$ category, there is a bijective correspondence between its right splittings and the isomorphisms with those semidirect product extensions. As a consequence, the 
discrete Atiyah sequence is right split in the $l L G p d$ category if and only if it is isomorphic to a semidirect product extension, thus giving yet another characterization of the flat discrete connections on $\pi$ (Corollary 5.59).

The plan for the paper is as follows. In Section 2 we review some background material regarding the continuous as well as the discrete Atiyah sequence, we also revisit the basic results about discrete connections on a principal $G$-bundle. In Section 3 we study the discrete Atiyah sequence of a principal bundle in the category Fbs of fiber bundles with a section, a category that we introduce in Section 3.1. The various bijective correspondences proved in this Section are summarized in Theorem 3.35. Lie groupoids and their morphisms are reviewed in Section 4, where we define the curvature of a discrete connection on a principal bundle. In Section 5 we study the discrete Atiyah sequence in the category of local Lie groupoids. The semidirect product of (some) local Lie groupoids is introduced in Section 5.5, where its relationship to split extensions in the $l L G p d$ category is established.

Notation: throughout the paper the action of a group $G$ on the set $X$ is denoted by $l^{X}$ and the corresponding quotient map by $\pi^{X, G}: X \rightarrow X / G$. In general, $\pi: Q \rightarrow Q / G$ is the principal $G$-bundle induced by the $G$-action $l^{Q}$ on $Q$. Given $f: X \rightarrow Y$ and subsets $X^{\prime} \subset X$ and $Y^{\prime} \subset Y$ the maps $\left.f\right|_{X^{\prime}}: X^{\prime} \rightarrow Y$ and, if $f(X) \subset Y^{\prime},\left.f\right|^{Y^{\prime}}: X \rightarrow Y^{\prime}$ are the restriction and co-restriction of $f$.

\section{Some BACKGround}

In this section we review some of the main objects we work with in the paper: the Atiyah sequence associated to a principal bundle and the discrete connections of those spaces. We also introduce the discrete version of the Atiyah sequence.

2.1. The Atiyah sequence. The Atiyah sequence was introduced by M. Atiyah in [Ati57] to study holomorphic connections on (holomorphic) principal bundles. The construction has been successfully applied to smooth objects too, as we review next. Later, in Section 2.1.2, we present the discrete analogue (in the smooth setting).

2.1.1. Continuous case. Given the principal $G$-bundle $\pi: Q \rightarrow Q / G$, we have the following sequence of vector bundles over $Q$ :

$$
0 \longrightarrow Q \times \mathfrak{g} \stackrel{\widehat{\phi_{1}}}{\longrightarrow} T Q \stackrel{\widehat{\phi_{2}}}{\longrightarrow} \pi^{*} T(Q / G) \longrightarrow 0
$$

where $\mathfrak{g}:=\operatorname{Lie}(G), \widehat{\phi_{1}}(q, \xi):=\xi_{Q}(q)=\left.\frac{d}{d t}\right|_{t=0} l_{\exp (t \xi)}^{Q}(q)$ and $\widehat{\phi_{2}}\left(v_{q}\right):=\left(q, T_{q} \pi\left(v_{q}\right)\right)$. It is easy to see that (2.1) is, indeed, an exact sequence of vector bundles over $Q$. If we now consider the $G$-actions $l_{g}^{Q \times \mathfrak{g}}(q, \xi):=\left(l_{g}^{Q}(q), A d_{g}(\xi)\right), l_{g}^{T Q}\left(v_{q}\right):=T_{q} l_{g}^{Q}\left(v_{q}\right)$ and $l_{g}^{\pi^{*} T(Q / G)}\left(q, r_{\pi(q)}\right):=\left(l_{g}^{Q}(q), r_{\pi(q)}\right)=\left(l_{g}^{Q}(q), r_{\pi\left(l_{g}^{Q}(q)\right)}\right)$, direct computations show that $\widehat{\phi_{1}}$ and $\widehat{\phi_{2}}$ are $G$-equivariant, thus we get a new sequence of vector bundles over $Q / G$ :

$$
0 \longrightarrow(Q \times \mathfrak{g}) / G \longrightarrow(T Q) / G \longrightarrow\left(\pi^{*} T(Q / G)\right) / G \longrightarrow 0
$$

The vector bundle $\widetilde{\mathfrak{g}}:=(Q \times \mathfrak{g}) / G$ is the adjoint bundle. Also, $\left(\pi^{*} T(Q / G)\right) / G$ and $T(Q / G)$ are isomorphic as vector bundles over $Q / G$ via $\pi^{\pi^{*} T(Q / G), G}\left(q, r_{\pi(q)}\right) \mapsto$ $r_{\pi(q)}$. Thus, the previous sequence is isomorphic to the sequence

$$
0 \longrightarrow \widetilde{\mathfrak{g}} \stackrel{\phi_{1}}{\longrightarrow}(T Q) / G \stackrel{\phi_{2}}{\longrightarrow} T(Q / G) \longrightarrow 0
$$

of vector bundles over $Q / G$ that is exact and is called the Atiyah sequence of $\pi$. Explicitly, $\phi_{1}\left(\pi^{Q \times \mathfrak{g}, G}(q, \xi)\right):=\pi^{T Q, G}\left(\xi_{Q}(q)\right)$ and $\phi_{2}\left(\pi^{T Q, G}\left(v_{q}\right)\right):=T_{q} \pi\left(v_{q}\right)$. 
The Atiyah sequence is closely related to connections on the principal $G$-bundle $\pi$. Indeed, Atiyah defines a connection as a splitting of (2.2) (Definition on page 188 of [Ati57]) and so does Mackenzie (Definition 4.1 in Appendix A of [Mac87]). A lift of the image of a right splitting of (2.2) to $T Q$ corresponds precisely to the horizontal distribution of such a connection, while its connection form is equivalent to a lift of a left splitting of (2.2) (see pp. 292-3 in [Mac87]).

At another level, the Atiyah sequence (2.2) can be interpreted as an exact sequence in the category of Lie algebroids. Not all the splittings described in the previous paragraph are still morphisms in this category. Only those that arise from flat connections correspond to right splittings of (2.2) in the category of Lie algebroids.

For more details on these subjects good references are the Appendix A of [Mac87], and [GKP11].

2.1.2. Discrete case. As we mentioned in the Introduction, the discrete analogue of $T Q$ that we consider is the space $Q \times Q$. Following ideas of [LMW05], next we introduce a discrete analogue of (2.2). A difference to notice is that while the objects that appear in (2.2) are vector bundles, their discrete analogues are fiber bundles and so, no linear structure is canonically available.

Definition 2.1. A fiber bundle $(E, \phi, M, S)$ consists of manifolds $E, M, S$ and a smooth map $\phi: E \rightarrow M$ such that, for each $m \in M$, there is an open subset $U \subset M$ with $m \in U$ and such that $\left.E\right|_{U}:=\phi^{-1}(U)$ is diffeomorphic to $U \times S$ by a fiber preserving diffeomorphism so that the following diagram is commutative.

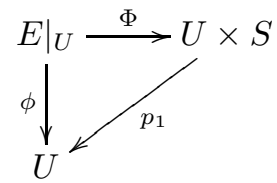

If $\left(E_{j}, \phi_{j}, M_{j}, S_{j}\right)$ (for $j=1,2$ ) are fiber bundles, a smooth map $F: E_{1} \rightarrow E_{2}$ is a bundle map over the smooth function $f: M_{1} \rightarrow M_{2}$ if the following diagram commutes.

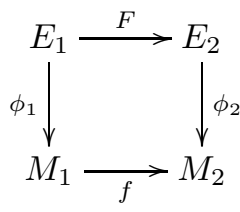

A section of the fiber bundle $(E, \phi, M, S)$ is a smooth function $\sigma: M \rightarrow E$ such that $\phi \circ \sigma=i d_{M}$. The set of all sections of $E$ is denoted by $\Gamma(E)$.

We notice that, by the local product structure, the projection map $\phi$ of a fiber bundle is a submersion. It is customary to refer to a fiber bundle $(E, \phi, M, S)$ just as $E$, something that we do in what follows unless it is necessary to provide all the data.

Inspired by (2.1), given a principal $G$-bundle $\pi: Q \rightarrow Q / G$ we consider the following sequence of fiber bundles over $Q$ (with the projection onto the first variable as bundle projection in the three cases)

$$
Q \times G \stackrel{\widehat{F_{1}}}{\longrightarrow} Q \times Q \stackrel{\widehat{F_{2}}}{\longrightarrow} Q Q_{\pi} \times_{p_{1}}((Q / G) \times(Q / G))
$$

where $\widehat{F_{1}}(q, g):=\left(q, l_{g}^{Q}(q)\right) \quad$ and $\quad \widehat{F_{2}}\left(q_{0}, q_{1}\right):=\left(q_{0},\left(\pi\left(q_{0}\right), \pi\left(q_{1}\right)\right)\right)$. 
Clearly $\widehat{F_{1}}$ is injective and $\widehat{F_{2}}$ is onto; $(2.3)$ is a sequence of fiber bundles and maps over $Q$. If we consider the $G$-actions

$$
\begin{aligned}
& l_{g}^{Q \times G}(q, h):=\left(l_{g}^{Q}(q), g h g^{-1}\right), \quad l_{g}^{Q \times Q}\left(q_{0}, q_{1}\right):=\left(l_{g}^{Q}\left(q_{0}\right), l_{g}^{Q}\left(q_{1}\right)\right), \\
& l_{g}^{Q_{\pi} \times p_{1}}((Q / G) \times(Q / G)) \\
& \left(q_{0},\left(\pi\left(q_{0}\right), \pi\left(q_{1}\right)\right)\right):=\left(l_{g}^{Q}\left(q_{0}\right),\left(\pi\left(q_{0}\right), \pi\left(q_{1}\right)\right)\right),
\end{aligned}
$$

both $\widehat{F_{1}}$ and $\widehat{F_{2}}$ are $G$-equivariant. Thus, from (2.3) we obtain the sequence

$$
(Q \times G) / G \longrightarrow(Q \times Q) / G \longrightarrow\left(Q_{\pi} \times_{p_{1}}((Q / G) \times(Q / G))\right) / G
$$

Notice that $\left(\pi\left(q_{0}\right), \pi\left(q_{1}\right)\right) \mapsto \pi^{Q_{\pi} \times_{p_{1}}((Q / G) \times(Q / G)), G}\left(q_{0},\left(\pi\left(q_{0}\right), \pi\left(q_{1}\right)\right)\right)$ is an isomorphism of fiber bundles over $Q / G$, from $(Q / G) \times(Q / G)$ onto $\left(Q_{\pi} \times{ }_{p_{1}}((Q / G) \times\right.$ $(Q / G))) / G$. Thus, the previous sequence produces the following sequence of fiber bundles over $Q / G$

$$
\begin{gathered}
\widetilde{G} \stackrel{F_{1}}{\longrightarrow}(Q \times Q) / G \stackrel{F_{2}}{\longrightarrow}(Q / G) \times(Q / G) \quad \text { for } \\
F_{1}\left(\pi^{Q \times G, G}(q, g)\right):=\pi^{Q \times Q, G}\left(q, l_{g}^{Q}(q)\right) \quad \text { and } \quad F_{2}\left(\pi^{Q \times Q, G}\left(q_{0}, q_{1}\right)\right):=\left(\pi\left(q_{0}\right), \pi\left(q_{1}\right)\right),
\end{gathered}
$$

where $\widetilde{G}:=(Q \times G) / G$ is the conjugate bundle - also known as the gauge bundleassociated to $Q$ over $Q / G$. By analogy with (2.2) we call sequence (2.5) the discrete Atiyah sequence of $\pi$.

While the Atiyah sequence (2.2) is a sequence of vector bundles or, even, of Lie algebroids over $Q / G$, so that notions as exactness or being split have a well defined meaning, the discrete Atiyah sequence (2.5) is a sequence of fiber bundles or, as we will discuss later, of Lie groupoids: in both cases, the categorical notions mentioned above are not available. The study of the discrete Atiyah sequences in these two contexts and their relationship to discrete connections and their curvature are the subject of this paper.

2.2. Discrete connections on principal fiber bundles. As we mentioned in the Introduction, discrete connections were introduced in [LMW05] and refined in [FZ13]. We follow this last work to which we refer the reader for further details. Let $\pi: Q \rightarrow Q / G$ be a principal $G$-bundle; we denote the associated left $G$-action on $Q$ by $l^{Q}$. We consider two $G$-actions on $Q \times Q$ : the diagonal action $l^{Q \times Q}$ (see $(2.4))$ and the action on the second component, $l_{g}^{Q \times Q_{2}}\left(q, q^{\prime}\right):=\left(q, l_{g}^{Q}\left(q^{\prime}\right)\right)$ for any $g \in G$. As usual, for any manifold $X, \Delta_{X} \subset X \times X$ is the diagonal submanifold and we define the discrete vertical submanifold $\mathcal{V}_{d}:=l_{G}^{Q \times Q_{2}}\left(\Delta_{Q}\right)=\left\{\left(q, l_{g}^{Q}(q)\right) \in\right.$ $Q \times Q: q \in Q$ and $g \in G\}$.

Definition 2.2. Let $H$ or $\subset Q \times Q$ be an $l^{Q \times Q}$-invariant embedded submanifold containing the diagonal $\Delta_{Q} \subset Q \times Q$. We say that Hor defines the discrete connection $\mathcal{A}_{d}$ on the principal bundle $\pi: Q \rightarrow Q / G$ if $\left.\left(i d_{Q} \times \pi\right)\right|_{H o r}: H o r \rightarrow Q \times(Q / G)$ is an injective local diffeomorphism. We denote Hor by $\operatorname{Hor}_{\mathcal{A}_{d}}$. If, $\mathrm{Hor}_{\mathcal{A}_{d}}$ is a discrete connection such that for all $\left(q, q^{\prime}\right) \in Q \times Q$ we have that $\left(q, q^{\prime}\right) \in H_{\text {or }} r_{\mathcal{A}_{d}} \Longleftrightarrow$ $\left(q^{\prime}, q\right) \in \operatorname{Hor}_{\mathcal{A}_{d}}$, we say that $\operatorname{Hor}_{\mathcal{A}_{d}}$ is symmetric.

Next we introduce several types of subset that will be very useful in what follows.

Definition 2.3. Let $\pi: Q \rightarrow Q / G$ be a principal $G$-bundle. An open subset

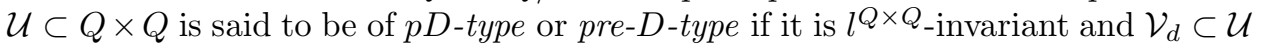
(in particular, $\Delta_{Q} \subset \mathcal{U}$ ). A $p D$-type subset $\mathcal{U}$ is of $D$-type if it is $G \times G$-invariant. If $\mathcal{U} \subset Q \times Q$ is of $D$-type (or $p D$-type) and is $\mathbb{Z}_{2}$-invariant for the standard action that interchanges components it is said to be of symmetric D-type (or symmetric $p D$-type). 
Since $\pi \times \pi$ and $i d_{Q} \times \pi$ are open maps, if $\mathcal{U} \subset Q \times Q$ is open (in particular, when $\mathcal{U}$ is a $p D$-type subset), then $\mathcal{U}^{\prime}:=\left(i d_{Q} \times \pi\right)(\mathcal{U}) \subset Q \times(Q / G)$ and $\mathcal{U}^{\prime \prime}:=$ $(\pi \times \pi)(\mathcal{U}) \subset(Q / G) \times(Q / G)$ are open subsets. We will use this notation in what follows.

Proposition 2.4. Let Hor $_{\mathcal{A}_{d}}$ be a discrete connection on the principal G-bundle $\pi: Q \rightarrow Q / G$. Then

$$
\mathfrak{U}:=l_{G}^{Q \times Q_{2}}\left(\text { Hor }_{\mathcal{A}_{d}}\right)=\left\{\left(q_{0}, l_{g}^{Q}\left(q_{1}\right)\right) \in Q \times Q:\left(q_{0}, q_{1}\right) \in \text { Hor }_{\mathcal{A}_{d}}, g \in G\right\} \subset Q \times Q
$$

is a D-type subset. If, in addition, Hor $_{\mathcal{A}_{d}}$ is symmetric, then $\mathfrak{U}$ is of symmetric D-type.

Proof. See Proposition 2.4 in [FZ13].

The open subset $\mathfrak{U}$ defined in Proposition 2.4 will be called the domain of the discrete connection $\operatorname{Hor}_{\mathcal{A}_{d}}$. For any $\left(q, q^{\prime}\right) \in \mathfrak{U}$ we have that there are $g \in G$ such that

$$
\left(q, q^{\prime}\right)=l_{g}^{Q \times Q_{2}}\left(q, q^{\prime \prime}\right) \quad \text { for some } \quad\left(q, q^{\prime \prime}\right) \in \text { Hor }_{\mathcal{A}_{d}}
$$

In fact, it is easy to see that such $g$ and $\left(q, q^{\prime \prime}\right)$ are unique.

Definition 2.5. The discrete connection form associated to the discrete connection $\operatorname{Hor}_{\mathcal{A}_{d}}$ is

$$
\mathcal{A}_{d}: \mathfrak{U} \rightarrow G \quad \text { with } \quad \mathcal{A}_{d}\left(q_{0}, q_{1}\right):=g
$$

where $g$ is the one given in (2.6). The symmetry of a discrete connection is equivalent to its domain $\mathfrak{U}$ being of symmetric $D$-type and its discrete connection form satisfying $\mathcal{A}_{d}\left(q_{0}, q_{1}\right)=\mathcal{A}_{d}\left(q_{1}, q_{0}\right)^{-1}$ for all $\left(q_{0}, q_{1}\right) \in \mathfrak{U}$. For a $D$-type subset $\mathcal{U} \subset Q \times Q$, we denote the set of all discrete connection forms with domain $\mathcal{U}$ by $\Sigma_{C}(\mathcal{U})$.

The following result states some properties of discrete connection forms that, in fact, completely characterize them.

Theorem 2.6. Let $\mathcal{A}_{d}$ be a discrete connection with domain $\mathfrak{U}$ on the principal $G$-bundle $\pi: Q \rightarrow Q / G$. Then, the discrete connection form $\mathcal{A}_{d}: \mathfrak{U} \rightarrow G$ is a smooth function such that, for all $\left(q_{0}, q_{1}\right) \in \mathfrak{U}$ and $g_{0}, g_{1} \in G$,

$$
\mathcal{A}_{d}\left(l_{g_{0}}^{Q}\left(q_{0}\right), l_{g_{1}}^{Q}\left(q_{1}\right)\right)=g_{1} \mathcal{A}_{d}\left(q_{0}, q_{1}\right) g_{0}^{-1} .
$$

In addition, Hor ${ }_{\mathcal{A}_{d}}=\left\{\left(q_{0}, q_{1}\right) \in \mathfrak{U}: \mathcal{A}_{d}\left(q_{0}, q_{1}\right)=e\right\}$. Conversely, given a D-type subset $\mathcal{U} \subset Q \times Q$ and a smooth function $\mathcal{A}: \mathcal{U} \rightarrow G$ such that (2.7) holds (with $\mathcal{A}_{d}$ replaced by $\left.\mathcal{A}\right)$ and $\mathcal{A}\left(q_{0}, q_{0}\right)=$ e for all $q_{0} \in Q$, then Hor $:=\left\{\left(q_{0}, q_{1}\right) \in \mathcal{U}:\right.$ $\left.\mathcal{A}\left(q_{0}, q_{1}\right)=e\right\}$ defines a discrete connection whose associated discrete connection form is $\mathcal{A}$.

Proof. Lemma 3.2 and Theorem 3.4 in [FZ13].

Definition 2.7. Let $\mathcal{U} \subset Q \times Q$ be of $p D$-type. A map $A: \mathcal{U} \rightarrow G$ is a pre-discrete connection form if, for all $g \in G, A \circ l_{g}^{Q \times Q}=l_{g}^{G} \circ A$ (for $l_{g}^{G}\left(g^{\prime}\right):=g g^{\prime} g^{-1}$ ) over $\mathcal{U}$ and $A\left(q, l_{g}^{Q}(q)\right)=g$ for all $q \in Q$. The set of all pre-discrete connection forms defined on $\mathcal{U}$ is denoted by $\Sigma_{C}^{\prime}(\mathcal{U})$. Clearly, when $\mathcal{U}$ is of $D$-type, $\Sigma_{C}(\mathcal{U}) \subset \Sigma_{C}^{\prime}(\mathcal{U})$.

Remark 2.8. The notion of pre-discrete connection form is, perhaps, implicit in [LMW05]. See, for instance, Proposition 4.3 as well as Lemma 4.10 (especially its proof).

When $\mathcal{A}_{d}$ is a discrete connection with domain $\mathfrak{U}$ on $\pi$, we can define $\mathfrak{U}^{\prime}:=\left(i d_{Q} \times\right.$ $\pi)(\mathfrak{U}) \subset Q \times(Q / G)$, that is an open subset and that $\left.\left(i d_{Q} \times \pi\right)\right|_{H_{\text {or }}{ }_{\mathcal{A}_{d}}}: H_{\text {or }_{\mathcal{A}_{d}}} \rightarrow \mathfrak{U}^{\prime}$ 
is a diffeomorphism. Its inverse map $h_{d}: \mathfrak{U}^{\prime} \rightarrow$ Hor $_{\mathcal{A}_{d}}$ is a diffeomorphism known as the discrete horizontal lift of $\mathcal{A}_{d}$. It is easy to check that

$$
h_{d}\left(q_{0}, \pi\left(q_{1}\right)\right)=\left(q_{0}, l_{\mathcal{A}_{d}\left(q_{0}, q_{1}\right)^{-1}}^{Q}\left(q_{1}\right)\right) \quad \text { for all } \quad\left(q_{0}, q_{1}\right) \in \mathfrak{U} .
$$

The next result establishes some of the properties of discrete horizontal lifts and, also, gives a complete characterization of discrete connections in their terms.

Theorem 2.9. Let $\mathcal{A}_{d}$ be a discrete connection with domain $\mathfrak{U}$ on the principal $G$-bundle $\pi: Q \rightarrow Q / G$. Then the following assertions are true.

(1) $\mathfrak{U}^{\prime} \subset Q \times(Q / G)$ is $G$-invariant for the $G$-action defined by $l_{g}^{Q \times(Q / G)}\left(q_{0}, r_{1}\right):=$ $\left(l_{g}^{Q}\left(q_{0}\right), r_{1}\right)$ for all $g \in G$.

(2) $h_{d}: \mathfrak{U}^{\prime} \rightarrow Q \times Q$ is smooth and $G$-equivariant for the $G$-actions $l^{Q \times(Q / G)}$ and $l^{Q \times Q}$.

(3) $h_{d}$ is a section over $\mathfrak{U}^{\prime}$ of $i d_{Q} \times \pi: Q \times Q \rightarrow Q \times(Q / G)$; that is, $\left(i d_{Q} \times\right.$ $\pi) \circ h_{d}=i d_{\mathfrak{U}^{\prime}}$.

(4) For every $q_{0} \in Q,\left(q_{0}, \pi\left(q_{0}\right)\right) \in \mathfrak{U}^{\prime}$ and $h_{d}^{q_{0}}\left(\pi\left(q_{0}\right)\right)=\left(q_{0}, q_{0}\right)$.

Conversely, assume that $\mathcal{U}^{\prime} \subset Q \times(Q / G)$ is an open set that satisfies condition 1 (with $\mathfrak{U}^{\prime}$ replaced by $\mathcal{U}^{\prime}$ ) and $h: \mathcal{U}^{\prime} \rightarrow Q \times Q$ is a map such that conditions 2, 3 and 4 are satisfied (with $\mathfrak{U}^{\prime}$ and $h_{d}$ replaced by $\mathcal{U}^{\prime}$ and $h$ ). Then, there exists a unique discrete connection $\mathcal{A}_{d}$ with domain $\mathfrak{U}:=\left(i d_{Q} \times \pi\right)^{-1}\left(\mathcal{U}^{\prime}\right)$ on $\pi: Q \rightarrow Q / G$ such that $\mathcal{U}^{\prime}=\mathfrak{U}^{\prime}$ and $h=h_{d}$.

Proof. Theorem 4.4 in [FZ13].

We denote the set of all discrete horizontal lifts defined on the set $\mathfrak{U}^{\prime}:=\left(i d_{Q} \times\right.$ $\pi)(\mathfrak{U})$ by $\Sigma_{H}(\mathfrak{U})$. The map $F_{C H}: \Sigma_{C}(\mathfrak{U}) \rightarrow \Sigma_{H}(\mathfrak{U})$ that assigns to a discrete connection form on $\mathfrak{U}$ its discrete horizontal lift over $\mathfrak{U}^{\prime}$ is a bijection by Theorems 2.6 and 2.9. We denote its inverse by $F_{H C}: \Sigma_{H}(\mathfrak{U}) \rightarrow \Sigma_{C}(\mathfrak{U})$.

\section{The Discrete Atiyah Sequence in the Category Fbs}

3.1. The category of fiber bundles with a section. It is well known that the smooth fiber bundles and the smooth bundle maps (together with the standard identity and composition of maps) form a category that we denote by $F b$. Let $M$ be a manifold, we denote by $F b_{M}$ the fiber category over $M$, that is, the subcategory of fiber bundles with base $M$ and morphisms over $i d_{M}$. Unfortunately, neither $F b$ nor $F b_{M}$ are abelian or exact categories, so there is no general notion of exactness or, even, of kernels. In order to recover some of those notions, we work in a slightly enriched version of the category $F b$.

We define the category of fiber bundles with a section, Fbs, as having objects

$\mathrm{ob}_{F} b s:=\left\{((E, \phi, M, S), \sigma):\right.$ such that $(E, \phi, M, S) \in \mathrm{ob}_{F} b$ and $\left.\sigma \in \Gamma(E)\right\}$,

and morphisms

$\operatorname{hom}_{F} b s\left(\left(E_{1}, \sigma_{1}\right),\left(E_{2}, \sigma_{2}\right)\right):=\left\{(F, f) \in \operatorname{hom}_{F} b\left(E_{1}, E_{2}\right)\right.$ such that $\left.F \circ \sigma_{1}=\sigma_{2} \circ f\right\}$.

It is easy to verify that, with the standard identity and composition of bundle maps, $F b s$ is indeed a category. For any smooth manifold $M$ we denote by $F b s_{M} \subset F b s$ the subcategory consisting of fiber bundles over $M$ and morphisms over $i d_{M}$; since all such morphisms are of the form $\left(F, i d_{M}\right)$ we refer to them just by $F$ in what follows.

Next we introduce some examples of fiber bundles with a section.

Example 3.1. Let $\pi: Q \rightarrow Q / G$ be a principal $G$-bundle. It is immediate that $\left(Q \times G, p_{1}, Q, G\right)$ and $\left(Q \times Q, p_{1}, Q, Q\right)$ are (trivial) fiber bundles over $Q$. Let $G$ act 
on $Q \times G$ by $l^{Q \times G}$ and on $Q \times Q$ by $l^{Q \times Q}$ (see (2.4)). Then, as both $p_{1}$ 's are $G$ equivariant, they induce smooth maps $\check{p_{1}}: \widetilde{G} \rightarrow Q / G$ and $\check{p_{1}}:(Q \times Q) / G \rightarrow Q / G$. It is easy to verify that $\left(\widetilde{G}, \check{p_{1}}, Q / G, G\right)$ and $\left((Q \times Q) / G, \check{p_{1}}, Q / G, Q\right)$ are fiber bundles (for instance, prove that $G$ acts on the original bundles in the sense of Definition 9.14 in [FTZ16] and, then, apply Proposition 9.16 of the same paper).We also have the trivial fiber bundle $p_{1}:(Q / G) \times(Q / G) \rightarrow Q / G$. Define

$$
\begin{array}{rll}
\sigma_{\widetilde{G}}: Q / G \rightarrow \widetilde{G} & \text { by } & \sigma_{\widetilde{G}}(\pi(q)):=\pi^{Q \times G, G}(q, e), \\
\sigma_{(Q \times Q) / G}: Q / G \rightarrow(Q \times Q) / G & \text { by } & \sigma_{(Q \times Q) / G}(\pi(q)):=\pi^{Q \times Q, G}(q, q), \\
\sigma_{Q / G \times Q / G}: Q / G \rightarrow(Q / G) \times(Q / G) & \text { by } & \sigma_{Q / G \times Q / G)}(\pi(q)):=(\pi(q), \pi(q)) .
\end{array}
$$

We have that $\sigma_{\widetilde{G}} \in \Gamma(\widetilde{G}), \sigma_{(Q \times Q) / G} \in \Gamma((Q \times Q) / G)$ and $\sigma_{Q / G \times Q / G} \in \Gamma(Q / G \times$ $Q / G)$. Hence $\left(\widetilde{G}, \sigma_{\widetilde{G}}\right),\left((Q \times Q) / G, \sigma_{(Q \times Q) / G}\right)$ and $\left(Q / G \times Q / G, \sigma_{Q / G \times Q / G}\right)$ are objects of $F b s_{Q / G}$. It is also easy to check that for $F_{1}$ and $F_{2}$ defined in (2.5),

$$
\begin{aligned}
& F_{1} \in \operatorname{hom}_{F b s_{Q / G}}\left(\left(\widetilde{G}, \sigma_{\widetilde{G}}\right),\left((Q \times Q) / G, \sigma_{(Q \times Q) / G}\right)\right), \\
& F_{2} \in \operatorname{hom}_{F b s_{Q / G}}\left(\left((Q \times Q) / G, \sigma_{(Q \times Q) / G}\right),\left(Q / G \times Q / G, \sigma_{Q / G \times Q / G}\right)\right) .
\end{aligned}
$$

Example 3.2. Let $M$ be a smooth manifold. Then, $\left(M, i d_{M}, M,\{0\}\right)$ is a (trivial) fiber bundle over $M$. Clearly, $i d_{M}$ is a section of that bundle. Hence,

$$
M^{\dagger}:=\left(\left(M, i d_{M}, M,\{0\}\right), i d_{M}\right) \in \mathrm{ob}_{F b s_{M}} .
$$

For $((E, \phi, M, S), \sigma) \in \mathrm{ob}_{F b s_{M}}$, define $0^{E}: M \rightarrow E$ by $0^{E}:=\sigma$ and $0_{E}: E \rightarrow$ $M$ by $0_{E}:=\phi$. It is easy to verify that $\operatorname{hom}_{F b s_{M}}\left(M^{\dagger},(E, \sigma)\right)=\left\{0^{E}\right\}$ and $\operatorname{hom}_{F b s_{M}}\left((E, \sigma), M^{\dagger}\right)=\left\{0_{E}\right\}$. An object in a category that has exactly one morphism to all objects in the category is called initial $^{2}$, while an object so that every object has exactly one morphism into it is called terminal; also, an object that is both initial and terminal in a category is called a zero object. Thus, $M^{\dagger}$ is a zero object in $F b s_{M}$.

Lemma 3.3. If $\pi: Q \rightarrow Q / G$ is a principal $G$-bundle and $F_{2}$ is defined by (2.5), then $F_{2}$ a a surjective submersion.

Proof. As $\pi: Q \rightarrow Q / G$ is a surjective submersion, the same thing happens to $\pi \times \pi: Q \times Q \rightarrow(Q / G) \times(Q / G)$. On the other hand, as $\pi^{Q \times Q, G}: Q \times Q \rightarrow(Q \times Q) / G$ is also a surjective submersion and $F_{2} \circ \pi^{Q \times Q, G}=\pi \times \pi$, the statement follows.

As $F b s_{M}$ is not an abelian category, the standard categorical notions of exact sequence and extension and their consequences are not naturally available. Still, in $F b s_{M}$, we consider the following naive notion, inspired by one that is in common use in the category of Lie groupoids (see Definition 5.4).

Definition 3.4. A sequence $\left(E_{1}, \sigma_{1}\right) \stackrel{\eta_{1}}{\longrightarrow}\left(E_{2}, \sigma_{2}\right) \stackrel{\eta_{2}}{\longrightarrow}\left(E_{3}, \sigma_{3}\right)$ in $F b s_{M}$ is said to be an extension of $\left(E_{3}, \sigma_{3}\right)$ by $\left(E_{1}, \sigma_{1}\right)$ if $\eta_{1}$ is an embedding, $\eta_{2}$ is onto, and the subsets $\operatorname{Im}\left(\eta_{1}\right):=\eta_{1}\left(E_{1}\right)$ and $\operatorname{ker}\left(\eta_{2}\right):=\eta_{2}^{-1}\left(\sigma_{3}(M)\right)$ are equal. An extension as above is said to be right split if $\eta_{2}$ has a right inverse and left split if $\eta_{1}$ has a left inverse.

Remark 3.5. If a sequence $\left(E_{1}, \sigma_{1}\right) \stackrel{\eta_{1}}{\longrightarrow}\left(E_{2}, \sigma_{2}\right) \stackrel{\eta_{2}}{\longrightarrow}\left(E_{3}, \sigma_{3}\right)$ in $F b s_{M}$ is an extension, it is easy to see that $\operatorname{Im}\left(\eta_{1}\right)$ is a fiber bundle over $M$ with the projection $\left.\phi_{2}\right|_{\operatorname{Im}\left(\eta_{1}\right)}$. Furthermore, as $\eta_{1} \in \operatorname{hom}_{F b s_{M}}\left(\left(E_{1}, \sigma_{1}\right),\left(E_{2}, \sigma_{2}\right)\right),\left(\left(\operatorname{Im}\left(\eta_{1}\right),\left.\sigma_{2}\right|^{\operatorname{Im}\left(\eta_{1}\right)}\right) \in\right.$ $\mathrm{ob}_{F b s_{M}}$. As $\operatorname{ker}\left(\eta_{2}\right)=\operatorname{Im}\left(\eta_{1}\right)$, it also follows that $\left(\operatorname{ker}\left(\eta_{2}\right),\left.\sigma_{2}\right|^{\operatorname{ker}\left(\eta_{2}\right)}\right) \in \mathrm{ob}_{F b s_{M}}$.

\footnotetext{
${ }^{2}$ We use a few simple categorical notions; [AHS06] could be used for further context.
} 
Example 3.6. By Example 3.1 we know that (2.5) is a sequence in $F b s_{Q / G}$. By Lemma 3.3, $F_{2}$ is onto. Next we see that $F_{1}$ is an embedding. Indeed, $\hat{F}_{1}: Q \times G \rightarrow$ $Q \times Q$ defined in (2.3) is an immersion and closed (essentially because the $G$-action on $Q$ is proper and, then, by a result of R. Palais [Pal70]), hence it is an embedding onto its image $\mathcal{V}_{d}=\hat{F}_{1}(Q \times G)$. As $\hat{F}_{1}$ is $G$-equivariant, it follows easily that the induced map in the quotient, $F_{1}: \widetilde{G} \rightarrow(Q \times Q) / G$, is an embedding. Last, notice that

$$
\operatorname{ker}\left(F_{2}\right)=F_{2}^{-1}\left(\sigma_{Q / G \times Q / G}(Q / G \times Q / G)\right)=l_{G}^{Q \times Q_{2}}\left(\Delta_{Q}\right) / G=\mathcal{V}_{d} / G
$$

Thus, as $\operatorname{Im}\left(F_{1}\right)=\mathcal{V}_{d} / G$, the discrete Atiyah sequence (2.5) is an extension in $\mathrm{Fbs}_{Q / G}$.

Remark 3.7. It may seem that the notions of kernel and extension are quite ad-hoc. Still, there are categorical notions that support these ideas. For example, in a category $\mathcal{C}$ with a zero object 0 , given a morphism $f \in \operatorname{hom}_{\mathcal{C}}\left(A_{1}, A_{2}\right)$ a pair $(K, j)$ where $j \in \operatorname{hom}_{\mathcal{C}}\left(K, A_{1}\right)$ is said to be a categorical kernel of $f$ if the sequence $0 \stackrel{0_{K}}{\longleftarrow} K \stackrel{j}{\rightarrow} A_{1}$ is a pullback of $0 \stackrel{0^{A_{2}}}{\longrightarrow} A_{2} \stackrel{f}{\leftarrow} A_{1}$, where $0_{K}$ and $0^{A_{2}}$ are the corresponding (unique) initial and final morphisms. As we saw in Example 3.2, $M^{\dagger}$ is a zero object in the $F b s_{M}$ category. Given a submersion $F \in \operatorname{hom}_{F b s_{M}}\left(\left(E_{1}, \sigma_{1}\right),\left(E_{2}, \sigma_{2}\right)\right)$ we can define $K:=F^{-1}\left(\sigma_{2}(M)\right) \subset E_{1}$ and $j: K \rightarrow E_{1}$. It is not hard to see that when $\left.\phi_{1}\right|_{K}: K \rightarrow M$ is a fiber bundle, $(K, j)$ is a categorical kernel in the previous sense. In addition, back in a category $\mathcal{C}$ as above, a sequence $A_{1} \stackrel{f_{1}}{\longrightarrow} A_{2} \stackrel{f_{2}}{\longrightarrow} A_{3}$ in $\mathcal{C}$ is said to be a categorical extension if $f_{2}$ is an epimorphism and $\left(A_{1}, f_{1}\right)$ is a categorical kernel of $f_{2}$. In the $F b s_{M}$ category it can be proved that an extension (Definition 3.4) is always a categorical extension. In particular, the discrete Atiyah sequence (2.5) is a categorical extension in $F b s_{M}$. Finally, we notice that a categorical extension $\left(E_{1}, \sigma_{1}\right) \stackrel{\eta_{1}}{\longrightarrow}\left(E_{2}, \sigma_{2}\right) \stackrel{\eta_{2}}{\longrightarrow}\left(E_{3}, \sigma_{3}\right)$ in $F b s_{M}$ where $\left.\phi_{2}\right|_{\eta_{2}^{-1}\left(\sigma_{3}(M)\right)}: \eta_{2}^{-1}\left(\sigma_{3}(M)\right) \rightarrow M$ is a fiber bundle is always an extension (Definition 3.4); one case where this condition is true is when $\eta_{2}$ is a proper submersion.

Sometimes we need to work with maps between objects of $F b s$ that are not globally defined. The following definition introduces a notion that is adequate in those cases.

Definition 3.8. Let $\left(E_{j}, \sigma_{j}\right) \in \mathrm{ob}_{F s_{M}}$ for $j=1,2, \mathcal{U} \subset E_{1}$ be open subset and $\Phi: \mathcal{U} \rightarrow E_{2}$ be a smooth map. $\Phi$ is called a semi-local morphism ${ }^{3}$ from $\left(E_{1}, \sigma_{1}\right)$ to $\left(E_{2}, \sigma_{2}\right)$ if

(1) $\phi_{2} \circ \Phi=\phi_{1} \mid \mathcal{U}$, where $\phi_{j}: E_{j} \rightarrow M$ are the fiber bundle projections, and

(2) $\sigma_{1}(M) \subset \mathcal{U}$ and $\Phi \circ \sigma_{1}=\sigma_{2}$.

We say that the semi-local morphism $\Phi$ is a semi-local isomorphism if there is a semi-local morphism $\Psi: \mathcal{W} \rightarrow E_{1}$ such that $\Psi \circ \Phi=i d_{\mathcal{U}}$ and $\Phi \circ \Psi=i d_{\mathcal{W}}$.

Lemma 3.9. Let $\left(E_{j}, \sigma_{j}\right) \in \mathrm{ob}_{F b s_{M}}$ for $j=1,2, \mathcal{U} \subset E_{1}$ be an open subset, $\Phi: \mathcal{U} \rightarrow E_{2}$ be a smooth map and $\eta \in \operatorname{hom}_{F b s_{M}}\left(\left(E_{2}, \sigma_{2}\right),\left(E_{1}, \sigma_{1}\right)\right)$.

(1) If $\eta \circ \Phi=i d_{\mathcal{U}}$ and condition 2 in Definition 3.8 hold or

(2) if $\Phi \circ \eta=i d_{\eta^{-1}(\mathcal{U})}, \sigma_{1}(M) \subset \mathcal{U}$ and condition 1 in Definition 3.8 hold, then $\Phi$ is a semi-local morphism from $\left(E_{1}, \sigma_{1}\right)$ to $\left(E_{2}, \sigma_{2}\right)$.

Proof. In the case of $1, \phi_{2} \circ \Phi=\left(\phi_{1} \circ \eta\right) \circ \Phi=\phi_{1} \circ i d_{\mathcal{U}}=\phi_{1} \mid \mathcal{U}$ so that condition 1 in Definition 3.8 holds. In the case of 2 , notice that, as $\eta\left(\sigma_{2}(M)\right)=\sigma_{1}(M) \subset \mathcal{U}$

\footnotetext{
${ }^{3}$ The reason we call these maps semi-local rather than just local is that they are defined in an open subset containing "the diagonal" $\sigma_{1}(M)$ rather than on any open subset.
} 
we have $\sigma_{2}(M) \subset \eta^{-1}(\mathcal{U})$ and, then, $\Phi \circ \sigma_{1}=\Phi \circ\left(\eta \circ \sigma_{2}\right)=i d_{\eta^{-1}(\mathcal{U})} \circ \sigma_{2}=\sigma_{2}$, so that condition 2 in Definition 3.8 holds. Therefore, in either case $\Phi$ is a semi-local morphism.

Definition 3.10. Let $\left(E_{j}, \sigma_{j}\right) \in \mathrm{ob}_{F b s_{M}}$ for $j=1,2, \eta \in \operatorname{hom}_{F b s_{M}}\left(\left(E_{2}, \sigma_{2}\right),\left(E_{1}, \sigma_{1}\right)\right)$ and $\Phi: \mathcal{U} \rightarrow E_{2}$ be a semi-local morphism from $\left(E_{1}, \sigma_{1}\right)$ to $\left(E_{2}, \sigma_{2}\right)$. We say that $\Phi$ is a semi-local left inverse of $\eta$ if $\left.\Phi \circ \eta\right|_{\eta^{-1}(\mathcal{U})}=i d_{\eta^{-1}(\mathcal{U})}$. Analogously, we say that $\Phi$ is a semi-local right inverse of $\eta$ if $\eta \circ \Phi=i d_{\mathcal{U}}$.

Definition 3.11. A semi-local left splitting of the sequence $\left(E_{1}, \sigma_{1}\right) \stackrel{\eta_{1}}{\longrightarrow}(E, \sigma) \stackrel{\eta_{2}}{\longrightarrow}$ $\left(E_{2}, \sigma_{2}\right)$ in $F b s_{M}$ with domain $\mathcal{U}$ is a semi-local left inverse of $\eta_{1}$ with domain $\mathcal{U}$. Similarly a semi-local right splitting of the same sequence with domain $\mathcal{U}$ is a semi-local right inverse of $\eta_{2}$ with domain $\mathcal{U}$.

3.2. Left splittings and discrete connections. In [LMW05], Section 4.6, it is discussed how discrete connections of $\pi: Q \rightarrow Q / G$ and left splittings of the discrete Atiyah sequence (2.5) are related. Here we want to revisit that analysis making explicit the categorical context.

Let $\mathcal{U} \subset Q \times Q$ be of $p D$-type. Define $\Sigma_{L}^{\prime}(\mathcal{U})$ as the set of all semi-local left splittings of the discrete Atiyah sequence (2.5) defined over $\mathcal{U} / G$ (for the $G$-action $\left.l^{Q \times Q}\right)$.

Given $A \in \Sigma_{C}^{\prime}(\mathcal{U})$ we define

$$
\widetilde{s_{L}}: \mathcal{U} \rightarrow Q \times G \quad \text { by } \widetilde{s_{L}}\left(q_{0}, q_{1}\right):=\left(q_{0}, A\left(q_{0}, q_{1}\right)\right)
$$

that is clearly smooth and $G$-equivariant (for the "diagonal" action and $l_{g}^{Q \times G}(q, h):=$ $\left.\left(l_{g}^{Q}(q), l_{g}^{G}(h)\right)\right)$, so that it defines a smooth map

$$
s_{L}: \mathcal{U} / G \rightarrow \widetilde{G} \quad \text { such that } \pi^{Q \times G, G} \circ \widetilde{s_{L}}=s_{L} \circ \pi^{Q \times Q, G} .
$$

Lemma 3.12. With the definitions as above, $s_{L} \in \Sigma_{L}^{\prime}(\mathcal{U})$.

Proof. Straightforward computations and use of option 2 of Lemma 3.9.

By Lemma 3.12

$$
F_{C^{\prime} L^{\prime}}: \Sigma_{C}^{\prime}(\mathcal{U}) \rightarrow \Sigma_{L}^{\prime}(\mathcal{U}) \quad \text { defined by } \quad F_{C^{\prime} L^{\prime}}(A):=s_{L}
$$

with $s_{L}$ as in (3.2) is well defined.

Next we introduce the auxiliary function

$$
\kappa_{2}: Q_{\pi} \times_{\check{p}_{1}} \widetilde{G} \rightarrow G \quad \text { such that } \quad \kappa_{2}\left(q, \pi^{Q \times G, G}\left(q^{\prime}, g^{\prime}\right)\right):=l_{\kappa\left(q, q^{\prime}\right)^{-1}}^{G}\left(g^{\prime}\right),
$$

where $\kappa\left(q, q^{\prime}\right):=h \in G$ if $l_{h}^{Q}(q)=q^{\prime}$. Notice that $\kappa_{2}$ is well defined because, for $h \in G$,

$$
l_{\kappa\left(q, l_{h}^{Q}\left(q^{\prime}\right)\right)^{-1}}^{G}\left(l_{h}^{G}\left(g^{\prime}\right)\right)=l_{\left(h \kappa\left(q, q^{\prime}\right)\right)^{-1}}^{G}\left(l_{h}^{G}\left(g^{\prime}\right)\right)=l_{\kappa\left(q, q^{\prime}\right)^{-1} h^{-1}}^{G}\left(l_{h}^{G}\left(g^{\prime}\right)\right)=l_{\kappa\left(q, q^{\prime}\right)^{-1}}^{G}\left(g^{\prime}\right) .
$$

Observe that

$$
\begin{aligned}
\kappa_{2}\left(l_{g}^{Q}(q), \pi^{Q \times G, G}\left(q^{\prime}, g^{\prime}\right)\right) & =l_{\kappa\left(l_{g}^{Q}(q), q^{\prime}\right)^{-1}}^{G}\left(g^{\prime}\right)=l_{\left(\kappa\left(q, q^{\prime}\right) g^{-1}\right)^{-1}}^{G}\left(g^{\prime}\right)=l_{g \kappa\left(q, q^{\prime}\right)^{-1}}^{G}\left(g^{\prime}\right) \\
& =l_{g}^{G}\left(l_{\kappa\left(q, q^{\prime}\right)^{-1}}^{G}\left(g^{\prime}\right)\right)=l_{g}^{G}\left(\kappa_{2}\left(q, \pi^{Q \times G, G}\left(q^{\prime}, g^{\prime}\right)\right)\right) \\
& =g \kappa_{2}\left(q, \pi^{Q \times G, G}\left(q^{\prime}, g^{\prime}\right)\right) g^{-1} .
\end{aligned}
$$

Given $s_{L} \in \Sigma_{L}^{\prime}(\mathcal{U})$ we define

$$
\hat{s_{L}}: \mathcal{U} \rightarrow Q_{\pi} \times_{\check{p}_{1}} \widetilde{G} \quad \text { by } \quad \hat{s_{L}}\left(q_{0}, q_{1}\right):=\left(q_{0}, s_{L}\left(\pi^{Q \times Q, G}\left(q_{0}, q_{1}\right)\right)\right)
$$

and

$$
A_{s_{L}}: \mathcal{U} \rightarrow G \quad \text { by } \quad A_{s_{L}}:=\kappa_{2} \circ \hat{s_{L}} \text {. }
$$

Being a composition of smooth functions, $A_{s_{L}}$ is smooth. 
Lemma 3.13. With the definitions as above, $A_{s_{L}} \in \Sigma_{C}^{\prime}(\mathcal{U})$.

Proof. Direct computation.

By Lemma 3.13 the map

$$
F_{L^{\prime} C^{\prime}}: \Sigma_{L}^{\prime}(\mathcal{U}) \rightarrow \Sigma_{C}^{\prime}(\mathcal{U}) \quad \text { defined by } \quad F_{L^{\prime} C^{\prime}}\left(s_{L}\right):=A_{s_{L}}
$$

with $A_{s_{L}}$ as in by (3.6) is well defined.

Proposition 3.14. The functions $F_{C^{\prime} L^{\prime}}$ and $F_{L^{\prime} C^{\prime}}$ defined by (3.3) and (3.7) are mutually inverses.

Proof. For any $s_{L} \in \Sigma_{L}^{\prime}(\mathcal{U})$, as $\phi_{\widetilde{G}} \circ s_{L}=\left.\phi_{(Q \times Q) / G}\right|_{\mathcal{U} / G}$, we can write, for any $\left(q_{0}, q_{1}\right) \in \mathcal{U}, s_{L}\left(\pi^{Q \times Q, G}\left(q_{0}, q_{1}\right)\right)=\pi^{Q \times G, G}\left(q_{0}, g\right)$ for some $g \in G$. Then, if $A_{s_{L}}:=$ $F_{L^{\prime} C^{\prime}}\left(s_{L}\right)$, we have that $A_{s_{L}}\left(q_{0}, q_{1}\right)=\kappa_{2}\left(q_{0}, s_{L}\left(\pi^{Q \times Q, G}\left(q_{0}, q_{1}\right)\right)\right)=\kappa_{2}\left(q_{0}, \pi^{Q \times G, G}\left(q_{0}, g\right)\right)=$ g. Now, if $\overline{s_{L}}:=F_{C^{\prime} L^{\prime}}\left(A_{s_{L}}\right)$, we have $\overline{s_{L}}\left(\pi^{Q \times Q, G}\left(q_{0}, q_{1}\right)\right)=\pi^{Q \times G, G}\left(q_{0}, A_{s_{L}}\left(q_{0}, q_{1}\right)\right)=$ $\pi^{Q \times G, G}\left(q_{0}, g\right)=s_{L}\left(\pi^{Q \times Q, G}\left(q_{0}, q_{1}\right)\right)$. Hence, $\overline{s_{L}}=s_{L}$ and, then, $F_{C^{\prime} L^{\prime}} \circ F_{L^{\prime} C^{\prime}}=$ $i d_{\Sigma_{L}^{\prime}(\mathcal{U})}$.

Conversely, given $A \in \Sigma_{C}^{\prime}(\mathcal{U})$, define $s_{L}:=F_{C^{\prime} L^{\prime}}(A)$ and $\bar{A}:=F_{L^{\prime} C^{\prime}}\left(s_{L}\right)$. Then, for $\left(q_{0}, q_{1}\right) \in \mathcal{U}$,

$$
\bar{A}\left(q_{0}, q_{1}\right)=\kappa_{2}\left(q_{0}, s_{L}\left(\pi^{Q \times Q, G}\left(q_{0}, q_{1}\right)\right)\right)=\kappa_{2}\left(q_{0}, \pi^{Q \times G, G}\left(q_{0}, A\left(q_{0}, q_{1}\right)\right)\right)=A\left(q_{0}, q_{1}\right)
$$

and we have that $\bar{A}=A$, so that $F_{L^{\prime} C^{\prime}} \circ F_{C^{\prime} L^{\prime}}=i d_{\Sigma_{C}^{\prime}(\mathcal{U})}$, concluding the proof.

So far, we have assumed that $\mathcal{U} \subset Q \times Q$ is of $p D$-type. Suppose that $\mathcal{U}$ is of $D$ type. The elements of $\Sigma_{C}(\mathcal{U})$ are precisely the discrete connection forms of discrete connections with domain $\mathcal{U}$ (on the fixed principal $G$-bundle $\pi: Q \rightarrow Q / G$ ). We define $\Sigma_{L}(\mathcal{U}):=F_{C^{\prime} L^{\prime}}\left(\Sigma_{C}(\mathcal{U})\right)$ and $F_{C L}: \Sigma_{C}(\mathcal{U}) \rightarrow \Sigma_{L}(\mathcal{U})$ as the appropriate restriction and co-restriction of $F_{C^{\prime} L^{\prime}}$; as $F_{C^{\prime} L^{\prime}}$ is a bijection, so is $F_{C L}$. Notice that if $F_{L C}: \Sigma_{L}(\mathcal{U}) \rightarrow \Sigma_{C}(\mathcal{U})$ is the corresponding restriction and co-restriction of $F_{L^{\prime} C^{\prime}}$, then $F_{L C}^{-1}=F_{C L}$.

Remark 3.15. For any $s_{L} \in \Sigma_{L}^{\prime}(\mathcal{U})$ we define

$$
\widetilde{s_{L}}: \mathcal{U} \rightarrow Q \times G \quad \text { by } \quad \widetilde{s_{L}}\left(q_{0}, q_{1}\right):=\left(q_{0}, \kappa_{2}\left(q_{0}, s_{L}\left(\pi^{Q \times Q, G}\left(q_{0}, q_{1}\right)\right)\right)\right)
$$

that, being a composition of smooth functions, is smooth. It is easy to verify that the diagram of manifolds and smooth maps

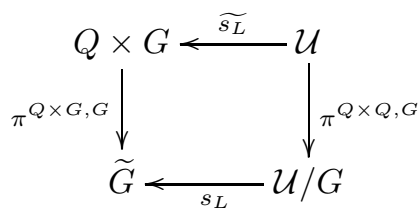

is commutative, so that $\widetilde{s_{L}}$ is a lift of $s_{L}$. When $\mathcal{U}$ is of $D$-type, we can consider the restriction of the $G$-action $l^{Q \times Q_{2}}$ to $\mathcal{U}$ and, also, the $G$-action $l_{g}^{Q \times G_{l m}}(q, h):=$ $(q, g h)$. It is then easy to check that $s_{L} \in \Sigma_{L}(\mathcal{U})$ if and only if $\widetilde{s_{L}}$ is $G$-equivariant for those actions.

Remark 3.16. Even for trivial principal $G$-bundles $\pi: Q \rightarrow Q / G$ with abelian $G$ it can be seen that $\Sigma_{L}(\mathcal{U}) \subsetneq \Sigma_{L}^{\prime}(\mathcal{U})$. 
3.3. Right splittings and horizontal liftings. Let $\pi: Q \rightarrow Q / G$ be a principal $G$-bundle, $\mathcal{U} \subset Q \times Q$ be a $D$-type open subset, $\mathcal{U}^{\prime}:=\left(i d_{Q} \times \pi\right)(\mathcal{U}) \subset Q \times(Q / G)$ and $\mathcal{U}^{\prime \prime}:=(\pi \times \pi)(\mathcal{U}) \subset(Q / G) \times(Q / G)$. In this section we study the relationship between discrete horizontal lifts of $\pi$ and semi-local right splittings of the discrete Atiyah sequence (2.5).

Before we tackle the real issues, we introduce two auxiliary functions. Define

$$
\tilde{\lambda}: Q_{\pi} \times_{\left(\pi \circ p_{1}\right)}(Q \times Q) \rightarrow Q \times Q \quad \text { by } \quad \tilde{\lambda}\left(q,\left(q_{0}, q_{1}\right)\right):=l_{\kappa\left(q_{0}, q\right)}^{Q \times Q}\left(q_{0}, q_{1}\right) .
$$

Let $l^{Q^{3}}$ be the $G$-action on $Q \times(Q \times Q)$ defined by $l_{g}^{Q^{3}}\left(q, q_{0}, q_{1}\right):=\left(q, l_{g}^{Q \times Q}\left(q_{0}, q_{1}\right)\right)$; then $Q_{\pi} \times{ }_{\left(\pi \circ p_{1}\right)}(Q \times Q)$ is a $G$-invariant closed submanifold, so $l^{Q^{3}}$ restricts to a $G$-action on $Q_{\pi} \times{ }_{\left(\pi \circ p_{1}\right)}(Q \times Q)$ that we still denote by $l^{Q^{3}}$. It is easy to see (for instance, using Proposition 9.1 in [FTZ16]), that $l^{Q^{3}}$ is a proper action on $Q^{3}$ and, as $Q_{\pi} \times{ }_{\left(\pi \circ p_{1}\right)}(Q \times Q)$ is a closed submanifold, its restriction to the submanifold is still proper; trivially, $l^{Q^{3}}$ is free, so its restriction to the same submanifold remains free. Notice that

$$
\begin{aligned}
\tilde{\lambda}\left(l_{g}^{Q^{3}}\left(q,\left(q_{0}, q_{1}\right)\right)\right) & =\widetilde{\lambda}\left(q,\left(l_{g}^{Q}\left(q_{0}\right), l_{g}^{Q}\left(q_{1}\right)\right)\right)=l_{\kappa\left(l_{g}^{Q}\left(q_{0}\right), q\right)}^{Q \times Q}\left(l_{g}^{Q \times Q}\left(q_{0}, q_{1}\right)\right) \\
& =l_{\kappa\left(q_{0}, q\right) g^{-1}}^{Q \times Q}\left(l_{g}^{Q \times Q}\left(q_{0}, q_{1}\right)\right)=l_{\kappa\left(q_{0}, q\right)}^{Q \times Q}\left(l_{g^{-1}}^{Q \times Q}\left(l_{g}^{Q \times Q}\left(q_{0}, q_{1}\right)\right)\right) \\
& =l_{\kappa\left(q_{0}, q\right)}^{Q \times Q}\left(q_{0}, q_{1}\right)=\tilde{\lambda}\left(q,\left(q_{0}, q_{1}\right)\right),
\end{aligned}
$$

so that $\widetilde{\lambda}$ is $G$-invariant and, then, by Corollary 9.5 of [FTZ16], $\widetilde{\lambda}$ induces a smooth map

$\lambda: Q_{\pi^{\circ}} \times_{\breve{p}_{1}}(Q \times Q) / G \rightarrow Q \times Q \quad$ such that $\quad \lambda\left(q, \pi^{Q \times Q, G}\left(q_{0}, q_{1}\right)\right)=\widetilde{\lambda}\left(q,\left(q_{0}, q_{1}\right)\right)$.

Notice that as, for $\left(q,\left(q_{0}, q_{1}\right)\right) \in Q^{3}$ and $g \in G$,

$$
\begin{aligned}
\widetilde{\lambda}\left(l_{g}^{Q}(q),\left(q_{0}, q_{1}\right)\right) & =l_{\kappa\left(q_{0}, l_{g}^{Q}(q)\right)}^{Q \times Q}\left(q_{0}, q_{1}\right)=l_{g \kappa\left(q_{0}, q\right)}^{Q \times Q}\left(q_{0}, q_{1}\right) \\
& =l_{g}^{Q \times Q}\left(l_{\kappa\left(q_{0}, q\right)}^{Q \times Q}\left(q_{0}, q_{1}\right)\right)=l_{g}^{Q \times Q}\left(\widetilde{\lambda}\left(q,\left(q_{0}, q_{1}\right)\right)\right),
\end{aligned}
$$

we have that, for $\left(q, \pi^{Q \times Q, G}\left(q_{0}, q_{1}\right)\right) \in Q_{\pi^{\circ}} \times_{\check{p}_{1}}(Q \times Q) / G$ and $g \in G$,

$$
\begin{aligned}
\lambda\left(l_{g}^{Q}(q), \pi^{Q \times Q, G}\left(q_{0}, q_{1}\right)\right) & =\widetilde{\lambda}\left(l_{g}^{Q}(q),\left(q_{0}, q_{1}\right)\right) \\
& =l_{g}^{Q \times Q}\left(\widetilde{\lambda}\left(q,\left(q_{0}, q_{1}\right)\right)\right)=l_{g}^{Q \times Q}\left(\lambda\left(q, \pi^{Q \times Q, G}\left(q_{0}, q_{1}\right)\right)\right),
\end{aligned}
$$

and we see that $\lambda$ is $G$-equivariant for the actions $l^{Q \times Q}$ and

$$
l_{g}^{Q \times(Q \times Q) / G}\left(q, \pi^{Q \times Q, G}\left(q_{0}, q_{1}\right)\right):=\left(l_{g}^{Q}(q), \pi^{Q \times Q, G}\left(q_{0}, q_{1}\right)\right) .
$$

Let $\Sigma_{R}(\mathcal{U})$ be the set of all semi-local right splittings of (2.5) defined over $\mathcal{U}^{\prime \prime}$. Then, for $s_{R} \in \Sigma_{R}(\mathcal{U})$, we define

$$
h: \mathcal{U}^{\prime} \rightarrow Q \times Q \quad \text { by } \quad h(q, r):=\lambda\left(q, s_{R}(\pi(q), r)\right) .
$$

Lemma 3.17. With the definitions as above, $h \in \Sigma_{H}(\mathcal{U})$.

Proof. Direct computations to check the hypotheses of Theorem 2.9.

We define the map

$$
F_{R H}: \Sigma_{R}(\mathcal{U}) \rightarrow \Sigma_{H}(\mathcal{U}) \quad \text { by } \quad F_{R H}\left(s_{R}\right):=h
$$

where $h$ is as in (3.11). $F_{R H}$ is well defined by Lemma 3.17.

Given $h \in \Sigma_{H}(\mathcal{U})$ we define

$$
\hat{s_{R}}: \mathcal{U}^{\prime} \rightarrow(Q \times Q) / G \quad \text { by } \quad \hat{s_{R}}(q, r):=\pi^{Q \times Q, G}(h(q, r)) \text {. }
$$


Being a composition of smooth functions, $\hat{s_{R}}$ is smooth and as, for any $g \in G$,

$$
\begin{aligned}
\hat{s_{R}}\left(l_{g}^{Q}(q), r\right) & =\pi^{Q \times Q, G}\left(h\left(l_{g}^{Q}\left(q_{0}\right), \pi\left(q_{1}\right)\right)\right)=\pi^{Q \times Q, G}\left(l_{g}^{Q \times Q}\left(h\left(q_{0}, \pi\left(q_{1}\right)\right)\right)\right) \\
& =\pi^{Q \times Q, G}\left(h\left(q_{0}, \pi\left(q_{1}\right)\right)\right)=\hat{s_{R}}(q, r),
\end{aligned}
$$

there is a unique smooth function

$$
s_{R}: \mathcal{U}^{\prime \prime} \rightarrow(Q \times Q) / G \quad \text { such that } \quad s_{R}(\pi(q), r)=\hat{s_{R}}(q, r) .
$$

Lemma 3.18. With the definitions as above, $s_{R} \in \Sigma_{R}(\mathcal{U})$.

Proof. Straightforward computations and use of option 1 of Lemma 3.9.

By Lemma 3.18, the map

$$
F_{H R}: \Sigma_{H}(\mathcal{U}) \rightarrow \Sigma_{R}(\mathcal{U}) \quad \text { such that } \quad F_{H R}(h):=s_{R}
$$

with $s_{R}$ as in (3.13) is well defined.

Proposition 3.19. The functions $F_{R H}$ and $F_{H R}$ defined by (3.12) and (3.14) are mutually inverse.

Proof. Given $s_{R} \in \Sigma_{R}(\mathcal{U})$, let $h:=F_{R H}\left(s_{R}\right)$ and $\overline{s_{R}}:=F_{H R}(h)$. For any $\left(q_{0}, q_{1}\right) \in$ $\mathcal{U}$, we have $s_{R}\left(\pi\left(q_{0}\right), \pi\left(q_{1}\right)\right)=\pi^{Q \times Q, G}\left(q_{0}^{\prime}, q_{1}^{\prime}\right)$ and, as $\phi_{(Q \times Q) / G} \circ s_{R}=\phi_{(Q / G) \times(Q / G)}$, we see that $\pi\left(q_{0}\right)=\pi\left(q_{0}^{\prime}\right)$, so that $s_{R}\left(\pi\left(q_{0}\right), \pi\left(q_{1}\right)\right)=\pi^{Q \times Q, G}\left(q_{0}, q_{1}^{\prime \prime}\right)$ for some $\left(q_{0}, q_{1}^{\prime \prime}\right) \in \mathcal{U}$. Then,

$$
\begin{aligned}
\overline{s_{R}}\left(\pi\left(q_{0}\right), \pi\left(q_{1}\right)\right) & =\pi^{Q \times Q, G}\left(h\left(q_{0}, \pi\left(q_{1}\right)\right)\right)=\pi^{Q \times Q, G}\left(\lambda\left(q_{0}, s_{R}\left(\pi\left(q_{0}\right), \pi\left(q_{1}\right)\right)\right)\right) \\
& =\pi^{Q \times Q, G}\left(\lambda\left(q_{0}, \pi^{Q \times Q, G}\left(q_{0}, q_{1}^{\prime \prime}\right)\right)\right)=\pi^{Q \times Q, G}\left(q_{0}, q_{1}^{\prime \prime}\right)=s_{R}\left(\pi\left(q_{0}\right), \pi\left(q_{1}\right)\right),
\end{aligned}
$$

so that $F_{H R} \circ F_{R H}=i d_{\Sigma_{R}(\mathcal{U})}$.

Given $h \in \Sigma_{H}(\mathcal{U})$, we define $s_{R}:=F_{H R}(h)$ and $\bar{h}:=F_{R H}\left(s_{R}\right)$. For any $\left(q_{0}, q_{1}\right) \in$ $\mathcal{U}$ there is $q_{1}^{\prime} \in Q$ such that $h\left(q_{0}, \pi\left(q_{1}\right)\right)=\left(q_{0}, q_{1}^{\prime}\right)$ and, then,

$$
\begin{aligned}
\bar{h}\left(q_{0}, \pi\left(q_{1}\right)\right) & =\lambda\left(q_{0}, s_{R}\left(\pi\left(q_{0}\right), \pi\left(q_{1}\right)\right)\right)=\lambda\left(q_{0}, \pi^{Q \times Q, G}\left(h\left(q_{0}, \pi\left(q_{1}\right)\right)\right)\right) \\
& =\lambda\left(q_{0}, \pi^{Q \times Q, G}\left(q_{0}, q_{1}^{\prime}\right)\right)=\left(q_{0}, q_{1}^{\prime}\right)=h\left(q_{0}, \pi\left(q_{1}\right)\right),
\end{aligned}
$$

and we conclude that $F_{R H} \circ F_{H R}=i d_{\Sigma_{H}(\mathcal{U})}$, which ends the proof of the statement.

Remark 3.20. By Proposition 3.19, when $\mathcal{U}$ is of $D$-type, there is a bijective correspondence between semi-local right splittings of (2.5) and discrete horizontal liftings on $\pi$ which, in turn, are equivalent to discrete connections on $\pi$. This equivalence is the discrete analogue of the equivalence between right splittings of the Atiyah sequence (2.2) and connections on $\pi$ as in [Mac87]. Notice that while right and left splittings of (2.2) are equivalent — and both are equivalent to connectionsin the discrete case an additional equivariance condition is required of a left splitting of (2.5) to correspond to a right splitting —or a discrete connection.

3.4. Fiber product isomorphism associated with a discrete connection. Let $\left(E_{j}, \phi_{j}, M, S_{j}, \sigma_{j}\right) \in \mathrm{ob}_{F b s_{M}}$ for $j=1,2$. As $\phi_{j}$ are submersions, it is easy to see that, $E_{\times}:=E_{1}{ }_{\phi_{1}} \times_{\phi_{2}} E_{2}$ is a manifold and, in fact, $E_{\times}$is a pullback of $E_{1} \stackrel{\phi_{1}}{\longrightarrow} M \stackrel{\phi_{2}}{\longleftarrow} E_{2}$ in the category of smooth manifolds. If we let $\phi_{\times}: E_{\times} \rightarrow M$ be such that $\phi_{\times}:=\phi_{1} \circ p_{1}^{r}=\phi_{2} \circ p_{2}^{r}$, it is easy to check that $\left(E_{\times}, \phi_{\times}, M, S_{1} \times S_{2}\right)$ is a fiber bundle and that, furthermore, if $\sigma_{\times}(m):=\left(\sigma_{1}(m), \sigma_{2}(m)\right)$, then $\left(E_{\times}, \sigma_{\times}\right) \in$ 
$\mathrm{ob}_{F b s_{M}}$. As $E_{\times} \subset E_{1} \times E_{2}$ is an embedded submanifold, we can define the following four smooth maps:

$$
\begin{array}{lcc}
F_{1}^{\times}: E_{1} \rightarrow E_{\times} & \text {by } & F_{1}^{\times}\left(e_{1}\right):=\left(e_{1}, \sigma_{2}\left(\phi_{1}\left(e_{1}\right)\right)\right), \\
F_{2}^{\times}: E_{\times} \rightarrow E_{2} & \text { by } & F_{2}^{\times}\left(e_{1}, e_{2}\right):=e_{2}, \\
s_{1}^{\times}: E_{\times} \rightarrow E_{1} & \text { by } & s_{1}^{\times}\left(e_{1}, e_{2}\right):=e_{1}, \\
s_{2}^{\times}: E_{2} \rightarrow E_{\times} & \text {by } & s_{2}^{\times}\left(e_{2}\right):=\left(\sigma_{1}\left(\phi_{2}\left(e_{2}\right)\right), e_{2}\right) .
\end{array}
$$

It is easy to check that $F_{1}^{\times}, F_{2}^{\times}, s_{1}^{\times}, s_{2}^{\times}$are morphisms in $F b s_{M}$ and that the relations

(3.15) $s_{1}^{\times} \circ F_{1}^{\times}=i d_{E_{1}}, \quad F_{2}^{\times} \circ s_{2}^{\times}=i d_{E_{2}}, \quad F_{2}^{\times} \circ F_{1}^{\times}=\sigma_{2} \circ \phi_{1}, \quad s_{1}^{\times} \circ s_{2}^{\times}=\sigma_{1} \circ \phi_{2}$ are satisfied. We then have the following diagram in $F b s_{M}$

$$
\left(E_{1}, \sigma_{1}\right) \underset{s_{1}^{\times}}{\stackrel{F_{1}^{\times}}{\rightleftarrows}}\left(E_{\times}, \sigma_{\times}\right) \underset{s_{1}^{\times}}{\stackrel{F_{2}^{\times}}{\rightleftarrows}}\left(E_{2}, \sigma_{2}\right)
$$

The sequence

$$
\left(E_{1}, \sigma_{1}\right) \stackrel{F_{1}^{\times}}{\longrightarrow}\left(E_{\times}, \sigma_{\times}\right) \stackrel{F_{2}^{\times}}{\longrightarrow}\left(E_{2}, \sigma_{2}\right)
$$

will be called the fiber product sequence of $\left(E_{1}, \sigma_{1}\right)$ and $\left(E_{2}, \sigma_{2}\right)$. It can be verified that $\left(E_{1}, \sigma_{1}\right) \stackrel{s_{1}^{\times}}{\longleftarrow}\left(E_{\times}, \sigma_{\times}\right) \stackrel{F_{2}^{\times}}{\longrightarrow}\left(E_{2}, \sigma_{2}\right)$ is a pullback of $\left(E_{1}, \sigma_{1}\right) \stackrel{\phi_{1}}{\longrightarrow}\left(M^{\dagger}, i d_{M}\right) \stackrel{\phi_{2}}{\longleftarrow}$ $\left(E_{2}, \sigma_{2}\right)$ in $F_{b s}$.

Also, the fiber product sequence is an extension in $F b s_{M}$. Indeed, $F_{2}^{\times}$is a surjective submersion and, as $F_{1}^{\times}$is a right inverse of the smooth map $s_{1}^{\times}$, Lemma 3.21 proves that $F_{1}^{\times}$is an embedding. Last, a direct verification shows that $\operatorname{Im}\left(F_{1}^{\times}\right)=$ $\operatorname{ker}\left(F_{2}^{\times}\right)$.

Lemma 3.21. Let $\phi: X \rightarrow Y$ be a smooth map, $V \subset Y$ an open subset and $\sigma: V \rightarrow X$ a smooth right inverse (section) over $V$ of $\phi$, that is, $\phi \circ \sigma=i d_{V}$. Then, $\sigma$ is an embedding.

Proof. It follows from $\phi \circ \sigma=i d_{V}$ that $\sigma$ is injective and, taking derivatives, that $\sigma$ is an immersion.

Let $U \subset V$; then, $\sigma(U)=\phi^{-1}(U) \cap \sigma(V)$. Indeed, $\sigma(U) \subset \sigma(V)$ and, as $\phi(\sigma(U))=U \Rightarrow \sigma(U) \subset \phi^{-1}(U)$, we have that $\sigma(U) \subset \phi^{-1}(U) \cap \sigma(V)$. Conversely, if $x \in \phi^{-1}(U) \cap \sigma(V)$, there is $y \in V$ such that $x=\sigma(y)$ and, then, $y=\phi(\sigma(y)) \phi(x) \in U$, implying that $x \in \sigma(U)$, so that $\phi^{-1}(U) \cap \sigma(V) \subset \sigma(U)$. All together, we have seen that $\sigma(U)=\phi^{-1}(U) \cap \sigma(V)$ for any subset $U \subset V$.

If $U \subset V$ is open (as $V \subset Y$ is open $\Rightarrow U \subset Y$ is open) and $\phi: X \rightarrow Y$ is continuous, $\phi^{-1}(U) \subset X$ is open and, then, $\sigma(U)=\phi^{-1}(U) \cap \sigma(V)$ is open in $\sigma(V)$ with the subspace (of $X$ ) topology. We have then that $\left.\sigma\right|^{\sigma(V)}: V \rightarrow \sigma V$ is an open map (with the subspace of $X$ topology on $\sigma(V)$ ); consequently, $\left.\sigma\right|^{\sigma(V)}: V \rightarrow \sigma(V)$ is a homeomorphism.

Being an immersion and a homeomorphism onto its image, $\sigma$ is an embedding.

Given an extension in $F b s_{M}$

$$
\left(E_{1}, \sigma_{1}\right) \stackrel{F_{1}}{\longrightarrow}(E, \sigma) \stackrel{F_{2}}{\longrightarrow}\left(E_{2}, \sigma_{2}\right)
$$

we want to study different relationships between semi-local morphisms from (3.18) into the fiber product sequence (3.17) and semi-local splittings of (3.18). Fix an 
open subset $\mathcal{U} \subset E$ containing $\sigma(M)$. Let $\Phi: \mathcal{U} \rightarrow E_{\times}$be a semi-local morphism that makes

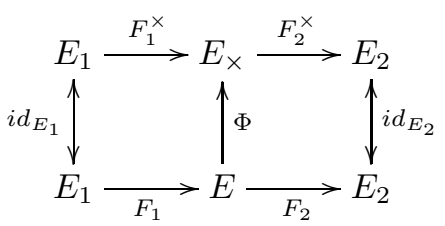

a commutative diagram in $F b s_{M}$. We define

$$
s_{1}: \mathcal{U} \rightarrow E_{1} \quad \text { by } \quad s_{1}:=s_{1}^{\times} \circ \Phi
$$

It is easy to check that $s_{1}$ is a semi-local morphism and we have $\left.s_{1} \circ F_{1}\right|_{F_{1}^{-1}(\mathcal{U})}=$ $\left.s_{1}^{\times} \circ \Phi \circ F_{1}\right|_{F_{1}^{-1}(\mathcal{U})}=\left.s_{1}^{\times} \circ F_{1}^{\times}\right|_{F_{1}^{-1}(\mathcal{U})}=i d_{F_{1}^{-1}(\mathcal{U})}$. Thus, $s_{1}$ is a semi-local left inverse of $F_{1}$ and, so, a semi-local left splitting of (3.18).

Conversely, given a semi-local left splitting $s_{1}: \mathcal{U} \rightarrow E_{1}$ of (3.18), we define

$$
\Phi: \mathcal{U} \rightarrow E_{\times} \quad \text { by } \quad \Phi:=\left(s_{1}, F_{2} \mid \mathcal{U}\right) .
$$

We check that $\Phi$ is well defined: for $e \in \mathcal{U}, \phi_{1}\left(s_{1}(e)\right)=\phi(e)=\phi_{2}\left(F_{2}(e)\right)$, so that $\Phi(e) \in E_{\times}$; also, $\Phi$ is smooth because it is a smooth map into $E_{1} \times E_{2}$ and $E_{\times} \subset E_{1} \times E_{2}$ is an embedded submanifold. We see that $\Phi$ is a semi-local morphism from $E$ into $E_{\times}$: first, $\sigma(M) \subset \mathcal{U}$ by hypothesis. Then, for $e \in \mathcal{U}$,

$$
\phi_{E_{\times}}(\Phi(e))=\phi_{E_{\times}}\left(s_{1}(e), F_{2}(e)\right)=\phi_{1}\left(s_{1}(e)\right)=\phi(e)
$$

and, for any $m \in M$,

$$
\Phi(\sigma(m))=\left(s_{1}(\sigma(m)), F_{2}(\sigma(m))\right)=\left(\sigma_{1}(m), \sigma_{2}(m)\right)=\sigma_{\times}(m) .
$$

We also have that $F_{2}^{\times} \circ \Phi=\left.F_{2}\right|_{\mathcal{U}}$ and, as $(3.18)$ is an extension, $\left(E_{1}, F_{1}\right)$ is a $\operatorname{ker}\left(F_{2}\right)$ so that the diagram

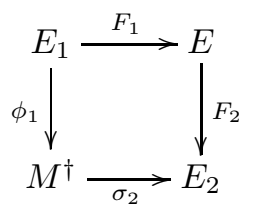

is commutative in $F b s_{M}$. Then, for any $e_{1} \in E_{1}, F_{2}\left(F_{1}\left(e_{1}\right)\right)=\sigma_{2}\left(\phi_{1}\left(e_{1}\right)\right)$. Thus,

$$
\Phi\left(F_{1}\left(e_{1}\right)\right)=\left(s_{1}\left(F_{1}\left(e_{1}\right)\right), F_{2}\left(F_{1}\left(e_{1}\right)\right)\right)=\left(e_{1}, \sigma_{2}\left(\phi_{1}\left(e_{1}\right)\right)\right)=F_{1}^{\times}\left(e_{1}\right) .
$$

All together, we conclude that, for $\Phi$ defined by (3.21), diagram (3.19) is commutative.

Proposition 3.22. For the extension (3.18) in the category Fbs ${ }_{M}$, the construction of the semi-local left splitting $s_{1}$ from a semi-local morphism $\Phi$ using (3.20) and the construction of the semi-local morphism $\Phi$ from the semi-local left splitting $s_{1}$ using (3.21) are mutually inverse operations.

Proof. Straightforward check.

We apply the previous constructions to $M=Q / G,\left(E_{1}, \sigma_{1}\right)=\left(\widetilde{G}, \sigma_{\widetilde{G}}\right)$ and $\left(E_{2}, \sigma_{2}\right)=\left((Q / G) \times(Q / G), \sigma_{(Q / G) \times(Q / G)}\right)$. Thus, we have the sequence in $F b s_{Q / G}$

$$
\widetilde{G} \underset{s_{1}^{\times}}{\stackrel{F_{1}^{\times}}{\rightleftarrows}} \widetilde{G} \underset{p_{1}}{\stackrel{F_{p_{1}}}{\rightleftarrows}}((Q / G) \times(Q / G)) \underset{s_{2}^{\times}}{\stackrel{F_{2}^{\times}}{\rightleftarrows}}(Q / G) \times(Q / G)
$$

The map $\Theta: \widetilde{G} \times(Q / G) \rightarrow \widetilde{G}_{\check{p_{1}}} \times_{p_{1}}((Q / G) \times(Q / G))$ defined by

$$
\Theta\left(\pi^{Q \times G, G}\left(q_{0}, g\right), \pi\left(q_{1}\right)\right):=\left(\pi^{Q \times G, G}\left(q_{0}, g\right),\left(\pi\left(q_{0}\right), \pi\left(q_{1}\right)\right)\right)
$$


is, actually, an isomorphism of fiber bundles over $Q / G$. If we define $\sigma_{\widetilde{G} \times(Q / G)}(\pi(q)):=$ $\left(\pi^{Q \times G, G}(q, e), \pi(q)\right)$, it is easy to verify that $\Theta$ is an isomorphism in $F b s_{Q / G}$. The previous sequence in $F b s_{Q / G}$ becomes

$$
\widetilde{G} \underset{\hat{s}_{1}}{\stackrel{\hat{F}_{1}}{\rightleftarrows}} \widetilde{G} \times(Q / G) \underset{\hat{s}_{2}}{\stackrel{\hat{F}_{2}}{\rightleftarrows}}(Q / G) \times(Q / G)
$$

where

$$
\begin{gathered}
\hat{F}_{1}\left(\pi^{Q \times G, G}(q, g)\right):=\left(\pi^{Q \times G, G}(q, g), \pi(q)\right), \quad \hat{F}_{2}\left(\pi^{Q \times G, G}(q, g), r\right):=(\pi(q), r), \\
\hat{s}_{1}\left(\pi^{Q \times G, G}(q, g), r\right):=\pi^{Q \times G, G}(q, g), \quad \hat{s}_{2}(\pi(q), r):=\left(\pi^{Q \times G, G}(q, e), r\right) .
\end{gathered}
$$

Sequence $(3.22)$ is the fiber product sequence of $\left(\widetilde{G}, \sigma_{\widetilde{G}}\right)$ with $\left((Q / G) \times(Q / G), \sigma_{(Q / G) \times(Q / G)}\right)$.

Going back to the existence of a discrete connection on a principal bundle, we quote the following result (Proposition 4.19 in [FTZ10]).

Proposition 3.23. Let $\mathcal{A}_{d}$ be a discrete connection with domain $\mathfrak{U}$ on the principal G-bundle $\pi: Q \rightarrow Q / G$. Define $\widetilde{\Phi}_{\mathcal{A}_{d}}: \mathfrak{U} \rightarrow Q \times G \times(Q / G)$ and $\widetilde{\Psi}_{\mathcal{A}_{d}}: \mathcal{W} \rightarrow Q \times Q$ where $\mathcal{W}:=\left\{\left(q_{0}, g, r_{1}\right) \in Q \times G \times(Q / G):\left(q_{0}, r_{1}\right) \in \mathfrak{U}^{\prime}\right\}$ by

$$
\begin{aligned}
\widetilde{\Phi}_{\mathcal{A}_{d}}\left(q_{0}, q_{1}\right) & :=\left(q_{0}, \mathcal{A}_{d}\left(q_{0}, q_{1}\right), \pi\left(q_{1}\right)\right), \\
\widetilde{\Psi}_{\mathcal{A}_{d}}\left(q_{0}, w_{0}, r_{1}\right) & :=l_{w_{0}}^{Q \times Q_{2}}\left(h_{d}\left(q_{0}, r_{1}\right)\right)
\end{aligned}
$$

Then $\widetilde{\Phi}_{\mathcal{A}_{d}}$ and $\widetilde{\Psi}_{\mathcal{A}_{d}}$ are smooth and mutually inverses. Furthermore, considering the diagonal action of $G$ on $Q \times Q$ and $l_{g}^{Q \times G \times(Q / G)}\left(q_{0}, w_{0}, r_{1}\right):=\left(l_{g}^{Q}\left(q_{0}\right), l_{g}^{G}\left(w_{0}\right), r_{1}\right)$, both maps are $G$-equivariant, so that they induce diffeomorphisms $\Phi_{\mathcal{A}_{d}}: \mathfrak{U} / G \rightarrow$ $\mathcal{W} / G$ and $\Psi_{\mathcal{A}_{d}}: \mathcal{W} / G \rightarrow \mathfrak{U} / G$.

Explicitly, the maps $\Phi_{\mathcal{A}_{d}}$ and $\Psi_{\mathcal{A}_{d}}$ are given by

$$
\begin{aligned}
\Phi_{\mathcal{A}_{d}}\left(\pi^{Q \times Q, G}\left(q_{0}, q_{1}\right)\right) & =\left(\pi^{Q \times G, G}\left(q_{0}, \mathcal{A}_{d}\left(q_{0}, q_{1}\right)\right), \pi\left(q_{1}\right)\right), \\
\Psi_{\mathcal{A}_{d}}\left(\pi^{Q \times G, G}\left(q_{0}, w_{0}\right), r_{1}\right) & =\pi^{Q \times Q, G}\left(l_{w_{0}}^{Q \times Q_{2}}\left(h_{d}\left(q_{0}, r_{1}\right)\right)\right) .
\end{aligned}
$$

It is easy to check that $\Phi_{\mathcal{A}_{d}}$ and $\Psi_{\mathcal{A}_{d}}$ are semi-local isomorphisms in $F b s_{Q / G}$. Furthermore, (ignoring the smaller domains of $\Phi_{\mathcal{A}_{d}}$ and $\Psi_{\mathcal{A}_{d}}$ ) the following diagrams are commutative.

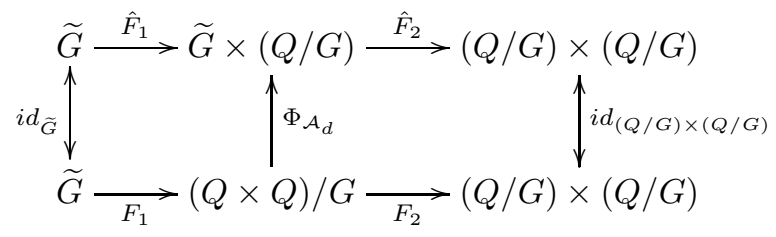

and

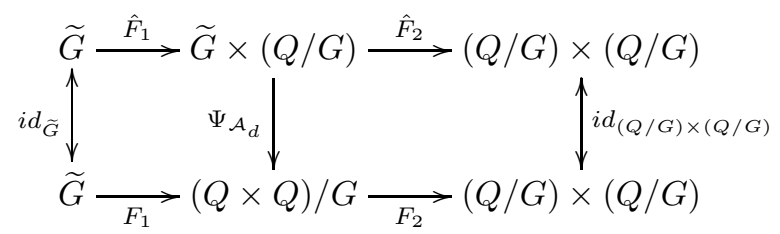

Corollary 3.24. Given a discrete connection $\mathcal{A}_{d}$ with domain $\mathfrak{U}$ on the principal $G$-bundle $\pi: Q \rightarrow Q / G$, the discrete Atiyah sequence (2.5) is semi-locally equivalent to a fiber product sequence (3.22) in $F b s_{Q / G}$ in a canonical way.

In the next two sections we explore how, semi-local equivalences in $F b s_{Q / G}$ between the discrete Atiyah sequence (2.5) and the fiber product sequence (3.22) may, in turn, define discrete connections on $\pi: Q \rightarrow Q / G$. 
3.5. Left splittings and fiber product decomposition. In this section we explore the relation between having a semi-local morphism $\Phi$ and a semi-local left splitting of the discrete Atiyah sequence.

Let $\mathcal{U} \subset Q \times Q$ be of $p D$-type and let $\Sigma_{U}^{\prime}(\mathcal{U})$ be the set of all semi-local morphisms $\Phi: \mathcal{U} / G \rightarrow \widetilde{G} \times(Q / G)$ that make the following diagram in $F b s_{Q / G}$ commutative (wherever it makes sense, because $\Phi$ is semi-local).

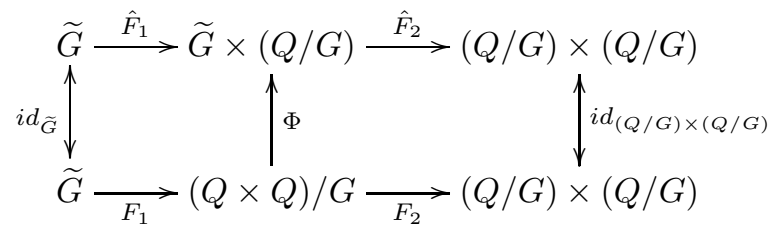

The constructions introduced in Section 3.4 can now be specialized to the current context to provide bijections between $\Sigma_{U}^{\prime}(\mathcal{U})$ and $\Sigma_{L}^{\prime}(\mathcal{U})$. Given $\Phi \in \Sigma_{U}^{\prime}(\mathcal{U})$, we define

$$
s_{L}: \mathcal{U} / G \rightarrow \widetilde{G} \quad \text { by } \quad s_{L}:=\hat{s}_{1} \circ \Phi .
$$

It was proved in Section 3.4 that $s_{L} \in \Sigma_{L}^{\prime}(\mathcal{U})$. Thus, we have defined a map

$$
F_{U^{\prime} L^{\prime}}: \Sigma_{U}^{\prime}(\mathcal{U}) \rightarrow \Sigma_{L}^{\prime}(\mathcal{U}) \quad \text { by } \quad F_{U^{\prime} L^{\prime}}(\Phi):=s_{L} .
$$

In the same spirit, given $s_{L} \in \Sigma_{L}^{\prime}(\mathcal{U})$ we define

$$
\Phi: \mathcal{U} / G \rightarrow \widetilde{G} \times(Q / G) \quad \text { by } \quad \Phi:=\left(s_{L},\left.p_{2} \circ F_{2}\right|_{\mathcal{U} / G}\right) .
$$

It was proved in Section 3.4 that $\Phi \in \Sigma_{U}^{\prime}(\mathcal{U})$, hence we can define a map

$$
F_{L^{\prime} U^{\prime}}: \Sigma_{L}^{\prime}(\mathcal{U}) \rightarrow \Sigma_{U}^{\prime}(\mathcal{U}) \quad \text { by } \quad F_{L^{\prime} U^{\prime}}\left(s_{L}\right):=\Phi .
$$

The following result is a direct specialization of Proposition 3.22.

Corollary 3.25. The functions $F_{U^{\prime} L^{\prime}}$ and $F_{L^{\prime} U^{\prime}}$ defined by (3.26) and (3.28) are mutually inverses.

When $\mathcal{U}$ is a $D$-type subset, the subset $\Sigma_{L}(\mathcal{U}) \subset \Sigma_{L}^{\prime}(\mathcal{U})$ corresponds to those semi-local left splittings that arise from discrete connections on $\pi$ with domain $\mathcal{U}$. Thus, $\Sigma_{U}(\mathcal{U}):=F_{L^{\prime} U^{\prime}}\left(\Sigma_{L}(\mathcal{U})\right)$ is the set of those semi-local morphisms in $\Sigma_{U}^{\prime}(\mathcal{U})$ that arise from a discrete connection on $\pi$ with domain $\mathcal{U}$. We define $F_{L U}$ : $\Sigma_{L}(\mathcal{U}) \rightarrow \Sigma_{U}(\mathcal{U})$ as the restriction and co-restriction of $F_{L^{\prime} U^{\prime}}$ to the corresponding subsets. It is clear that $F_{L U}$ is still a bijection and that, if $F_{U L}: \Sigma_{U}(\mathcal{U}) \rightarrow \Sigma_{L}(\mathcal{U})$ is the corresponding restriction and co-restriction of $F_{U^{\prime} L^{\prime}}$, then $F_{L U}^{-1}=F_{U L}$.

Remark 3.26. Assume that $\mathcal{U}$ is of $D$-type. For any $\Phi \in \Sigma_{U}^{\prime}(\mathcal{U})$, let $s_{L}:=$ $F_{U^{\prime} L^{\prime}}(\Phi)$ and $\widetilde{s_{L}}$ be the lift defined by (3.8). Then, $s_{L} \in \Sigma_{L}(\mathcal{U})$ if and only if $\widetilde{s_{L}}$ is $G$-equivariant in the sense of Remark 3.15. Define

$$
\widetilde{\Phi}: \mathcal{U} \rightarrow(Q \times G) \times(Q / G) \quad \text { by } \quad \widetilde{\Phi}\left(q_{0}, q_{1}\right):=\left(\widetilde{s_{L}}\left(q_{0}, q_{1}\right), \pi\left(q_{1}\right)\right) .
$$

Clearly $\widetilde{\Phi}$ is smooth and it is easy to check that the diagram

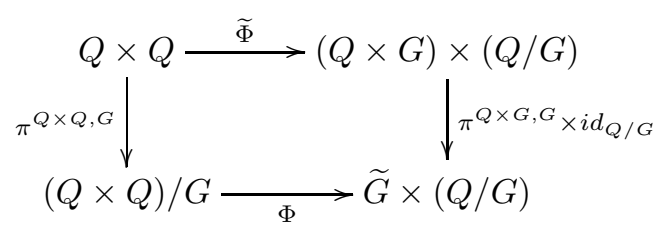

in the category of manifolds and smooth maps is commutative. Hence, in a sense, $\widetilde{\Phi}$ is a lift of $\Phi$. It can be checked that the condition of $\widetilde{s_{L}}$ being $G$-equivariant is equivalent to the $G$-equivariance of $\widetilde{\Phi}$ for the $G$-actions $l^{Q \times Q_{2}}$ and $l_{g}^{Q \times G_{l m} \times(Q / G)}(q, h, r):=$ 
$(q, g h, r)$. Thus, $\Phi \in \Sigma_{U}(\mathcal{U})$ if and only if its lift $\widetilde{\Phi}$ is $G$-equivariant for the actions just considered.

Proposition 3.27. If $\mathcal{U}$ is of D-type and $\Phi \in \Sigma_{U}(\mathcal{U})$, then $\Phi$ is a semi-local isomorphism in $\mathrm{Fbs}_{Q / G}$.

Proof. Let $\widetilde{\Phi}$ be the lift of $\Phi$, defined by $(3.29)$, where $s_{L}:=F_{U L}(\Phi)$. If the corresponding discrete connection form is $\mathcal{A}_{d}:=F_{L C}\left(s_{L}\right)$, using (3.1), we have that, for $\left(q_{0}, q_{1}\right) \in \mathcal{U}, \widetilde{\Phi}\left(q_{0}, q_{1}\right)=\left(\left(q_{0}, \mathcal{A}_{d}\left(q_{0}, q_{1}\right)\right), \pi\left(q_{1}\right)\right)$. As $\mathcal{A}_{d} \in \Sigma_{C}(\mathcal{U})$, it follows from Proposition 3.23 that $\Phi$ and $\widetilde{\Phi}$ are semi-local isomorphisms.

3.6. Right splittings and fiber product decomposition. In this section we explore the relation between having a semi-local morphism $\Psi$ and a semi-local right splitting of the discrete Atiyah sequence.

Let $\mathcal{U} \subset Q \times Q$ be a $D$-type subset; we define open subsets $\mathcal{U}^{\prime \prime}:=(\pi \times \pi)(\mathcal{U}) \subset$ $(Q / G) \times(Q / G)$ and $\mathcal{W}:=\hat{F}_{2}^{-1}\left(\mathcal{U}^{\prime \prime}\right)$. Let $\Sigma_{D}^{\prime}(\mathcal{U})$ be the set of all semi-local morphisms $\Psi: \mathcal{W} \rightarrow(Q \times Q) / G$ such that the following diagram in $F b s_{Q / G}$ is commutative.

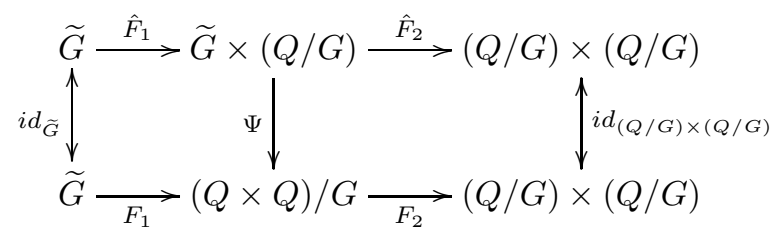

For $s_{R} \in \Sigma_{R}(\mathcal{U})$ we define

$$
\Psi: \mathcal{W} \rightarrow(Q \times Q) / G \quad \text { by } \quad \Psi\left(\pi^{Q \times G, G}(q, g), r\right):=\pi^{Q \times Q, G}\left(l_{g}^{Q \times Q_{2}}\left(\lambda\left(q, s_{R}(\pi(q), r)\right)\right)\right)
$$

where $\lambda$ is defined in (3.9).

Lemma 3.28. With the definitions as above, $\Psi \in \Sigma_{D}^{\prime}(\mathcal{U})$.

Proof. Lengthy but straightforward verification.

By Lemma 3.28, the following map is well defined.

$$
F_{R D^{\prime}}: \Sigma_{R}(\mathcal{U}) \rightarrow \Sigma_{D}^{\prime}(\mathcal{U}) \quad \text { so that } \quad F_{R D^{\prime}}\left(s_{R}\right):=\Psi,
$$

where $\Psi$ is defined by (3.31).

Given $\Psi \in \Sigma_{D}^{\prime}(\mathcal{U})$, we define

$$
s_{R}: \mathcal{U}^{\prime \prime} \rightarrow(Q \times Q) / G \quad \text { by } \quad s_{R}:=\Psi \circ \hat{s}_{2} \mid \mathcal{U}^{\prime \prime} .
$$

Lemma 3.29. With the definitions as above, $s_{R} \in \Sigma_{R}(\mathcal{U})$.

Proof. Direct computation and application of option 1 in Lemma 3.9.

By Lemma 3.29, the following function is well defined.

$$
F_{D^{\prime} R}: \Sigma_{D}^{\prime}(\mathcal{U}) \rightarrow \Sigma_{R}(\mathcal{U}) \quad \text { such that } \quad F_{D^{\prime} R}(\Psi):=s_{R}
$$

where $s_{R}$ is defined by $(3.33)$.

Proposition 3.30. For $\mathcal{U} \subset Q \times Q$ of D-type, the map $F_{D^{\prime} R}$ defined by (3.34) is a left inverse of $F_{R D^{\prime}}$ defined in (3.32). Consequently, $F_{R D^{\prime}}$ is one-to-one. 
Proof. For $s_{R} \in \Sigma_{R}(\mathcal{U})$, let $\Psi:=F_{R D^{\prime}}\left(s_{R}\right)$ and $\overline{s_{R}}:=F_{D^{\prime} R}(\Psi)$. Then, for $\left(q_{0}, q_{1}\right) \in$ $\mathcal{U}$, we have

$$
\begin{aligned}
\overline{s_{R}}\left(\pi\left(q_{0}\right), \pi\left(q_{1}\right)\right) & =\Psi\left(\hat{s}_{2}\left(\pi\left(q_{0}\right), \pi\left(q_{1}\right)\right)\right)=\Psi\left(\pi^{Q \times G, G}\left(q_{0}, e\right), \pi\left(q_{1}\right)\right) \\
& =\pi^{Q \times Q, G}\left(l_{e}^{Q \times Q_{2}}\left(\lambda(q_{0}, \underbrace{s_{R}\left(\pi\left(q_{0}\right), \pi\left(q_{1}\right)\right)}_{\pi_{R}^{Q \times Q, G}\left(q_{0}, q_{1}^{\prime}\right)})\right)\right. \\
& =\pi^{Q \times Q, G}\left(q_{0}, q_{1}^{\prime}\right)=s_{R}\left(\pi\left(q_{0}\right), \pi\left(q_{1}\right)\right),
\end{aligned}
$$

so that $\overline{s_{R}}=s_{R}$ and, then, $F_{D^{\prime} R} \circ F_{R D^{\prime}}=i d_{\Sigma_{R}(\mathcal{U})}$.

Remark 3.31. With the previous definitions, $F_{R D^{\prime}}: \Sigma_{R}(\mathcal{U}) \rightarrow \Sigma_{D}^{\prime}(\mathcal{U})$ may not be onto. Indeed, it is possible to find counterexamples when $\pi: Q \rightarrow Q / G$ is a trivial principal $G$-bundle with abelian $G$.

Let $\Sigma_{D}(\mathcal{U}):=F_{R D^{\prime}}\left(\Sigma_{R}(\mathcal{U})\right) \subset \Sigma_{D}^{\prime}(\mathcal{U})$. As, by Proposition 3.30, $F_{R D^{\prime}}$ is one-toone, its co-restriction $F_{R D}: \Sigma_{R}(\mathcal{U}) \rightarrow \Sigma_{D}(\mathcal{U})$ to $\Sigma_{D}(\mathcal{U})$ is a bijection.

Let $\Psi \in \Sigma_{D}^{\prime}(\mathcal{U})$ and define $\hat{\mathcal{W}}:=\left(\pi^{Q \times G, G} \times i d_{Q / G}\right)^{-1}(\mathcal{W}) \subset(Q \times G) \times(Q / G)$ for, as before, $\mathcal{W}:=\hat{F}_{2}^{-1}\left(\mathcal{U}^{\prime \prime}\right)$. In addition, define

$$
\hat{\Psi}: \hat{\mathcal{W}} \rightarrow Q \times Q \quad \text { by } \quad \hat{\Psi}(q, g, r):=\lambda\left(q, \Psi\left(\pi^{Q \times G, G}(q, g), r\right)\right)
$$

where $\lambda$ is defined by (3.9). As $\mathcal{W}$ is the domain of $\Psi$ and $\left(\pi^{Q \times G, G} \times i d_{Q / G}\right)(\hat{\mathcal{W}}) \subset$ $\mathcal{W}$, we see that $\Psi\left(\pi^{Q \times G, G}(q, g), r\right)$ is well defined for $\left(\pi^{Q \times G, G}(q, g), r\right) \in \hat{\mathcal{W}}$. Also, as $\Psi$ is a semi-local morphism,

$$
\begin{aligned}
\check{p_{1}}\left(\Psi\left(\pi^{Q \times G, G}(q, g), r\right)\right) & =\phi_{(Q \times Q) / G}\left(\Psi\left(\pi^{Q \times G, G}(q, g), r\right)\right) \\
& =\phi_{\widetilde{G} \times(Q / G)}\left(\pi^{Q \times G, G}(q, g), r\right)=\pi(q)
\end{aligned}
$$

and the composition with $\lambda$ is well defined. All together, $\hat{\Psi}$ is well defined and, being the composition of smooth functions, $\hat{\Psi}$ is smooth.

Lemma 3.32. For $\Psi \in \Sigma_{D}^{\prime}(\mathcal{U})$ and $\hat{\Psi}$ defined by (3.35), the following diagram in the category of smooth manifolds is commutative.

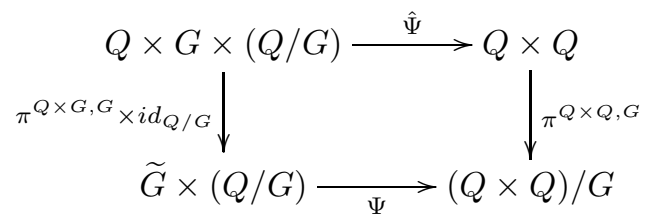

Proof. For $(q, g, r) \in \hat{\mathcal{W}}$ we can write $\Psi\left(\pi^{Q \times G, G}(q, g), r\right):=\pi^{Q \times Q, G}\left(q, q^{\prime}\right)$ for some $q^{\prime} \in Q$. Then

$$
\begin{aligned}
\pi^{Q \times Q, G}(\hat{\Psi}(q, g, r)) & =\pi^{Q \times Q, G}\left(\lambda\left(q, \Psi\left(\pi^{Q \times G, G}(q, g), r\right)\right)\right) \\
& =\pi^{Q \times Q, G}\left(\lambda\left(q, \pi^{Q \times Q, G}\left(q, q^{\prime}\right)\right)\right) \\
& =\pi^{Q \times Q, G}\left(q, q^{\prime}\right)=\Psi\left(\pi^{Q \times G, G}(q, g), r\right),
\end{aligned}
$$

proving the commutativity of the diagram.

Proposition 3.33. For $\Psi \in \Sigma_{D}^{\prime}(\mathcal{U})$, we have that $\Psi \in \Sigma_{D}(\mathcal{U})$ if and only if $\hat{\Psi}$ is $G$-equivariant for the actions $l_{g}^{Q \times G_{m l} \times(Q / G)}(q, h, r):=(q, g h, r)$ and $l^{Q \times Q_{2}}$.

Proof. If $\Psi \in \Sigma_{D}(\mathcal{U})$, then there is $s_{R} \in \Sigma_{R}(\mathcal{U})$ such that $\Psi=F_{R D^{\prime}}\left(s_{R}\right)$. A direct computation shows that, for $(q, h, r) \in \hat{\mathcal{W}}$ and $g \in G$

$$
\hat{\Psi}\left(l_{g}^{Q \times G_{l m} \times(Q / G)}(q, h, r)\right)=l_{g}^{Q \times Q_{2}}(\hat{\Psi}(q, h, r)),
$$

so that $\hat{\Psi}$ has the required $G$-equivariance. 
Conversely, assume that $\hat{\Psi}$ is $G$-equivariant for the given actions. Then, let $s_{R}:=F_{D^{\prime} R}(\Psi) \in \Sigma_{R}(\mathcal{U})$ and $\bar{\Psi}:=F_{R D^{\prime}}\left(s_{R}\right) \in \Sigma_{D}(\mathcal{U})$. We want to check that $\Psi=\bar{\Psi}$ so that $\Psi \in \Sigma_{D}(\mathcal{U})$. For any $(q, g, r) \in \hat{\mathcal{W}}$, using the $G$-equivariance of $\widetilde{\Psi}$ and the commutativity of (3.36), we have

$$
\begin{aligned}
\bar{\Psi}\left(\pi^{Q \times G, G}(q, g), r\right) & =\pi^{Q \times Q, G}\left(l_{g}^{Q \times Q_{2}}\left(\lambda\left(q, s_{R}(\pi(q), r)\right)\right)\right) \\
& =\pi^{Q \times Q, G}\left(l_{g}^{Q \times Q_{2}}\left(\lambda\left(q, \Psi\left(\pi^{Q \times G, G}(q, e), r\right)\right)\right)\right) \\
& =\pi^{Q \times Q, G}\left(l_{g}^{Q \times Q_{2}}(\hat{\Psi}(q, e, r))\right)=\pi^{Q \times Q, G}(\hat{\Psi}(q, g, r)) \\
& =\Psi\left(\pi^{Q \times G, G}(q, g), r\right),
\end{aligned}
$$

proving that $\Psi=\bar{\Psi}$, so that $\Psi \in \Sigma_{D}(\mathcal{U})$.

Remark 3.34. All elements of $\Sigma_{D}(\mathcal{U})$ are semi-local isomorphisms. Indeed, if $\Psi \in \Sigma_{D}(\mathcal{U})$, unraveling the definitions, if we let $s_{R}:=F_{D R}(\Psi), h:=F_{R H}\left(s_{R}\right)$ and $\mathcal{A}_{d}:=F_{H C}(h)$, we can write

$$
\begin{aligned}
\Psi\left(\pi^{Q \times G}\left(q_{0}, g\right), \pi\left(q_{1}\right)\right) & =\pi^{Q \times Q, G}\left(l_{g}^{Q \times Q_{2}}\left(\lambda\left(q_{0}, s_{R}\left(\pi\left(q_{0}\right), \pi\left(q_{1}\right)\right)\right)\right)\right) \\
& =\pi^{Q \times Q, G}\left(l_{g}^{Q \times Q_{2}}\left(\lambda\left(q_{0}, \pi^{Q \times Q, G}\left(h\left(q_{0}, \pi\left(q_{1}\right)\right)\right)\right)\right)\right. \\
& =\pi^{Q \times Q, G}\left(l_{g}^{Q \times Q_{2}}\left(h\left(q_{0}, \pi\left(q_{1}\right)\right)\right)\right) .
\end{aligned}
$$

Comparison of this last expression with (3.23) and the use of Proposition 3.23 proves that $\Psi$ is a semi-local isomorphism. In addition, it proves that $\Psi^{-1}=\Phi \in \Sigma_{U}(\mathcal{U})$, for $\Phi:=\left(F_{L U} \circ F_{C L}\right)\left(\mathcal{A}_{d}\right)$.

The next statement summarizes the results of this section concerning the discrete Atiyah sequence of a principal bundle and the discrete connections on the same space.

Theorem 3.35. Let $\pi: Q \rightarrow Q / G$ be a principal $G$-bundle and $\mathcal{U} \subset Q \times Q$ of $D$-type. Then we have the commutative diagram

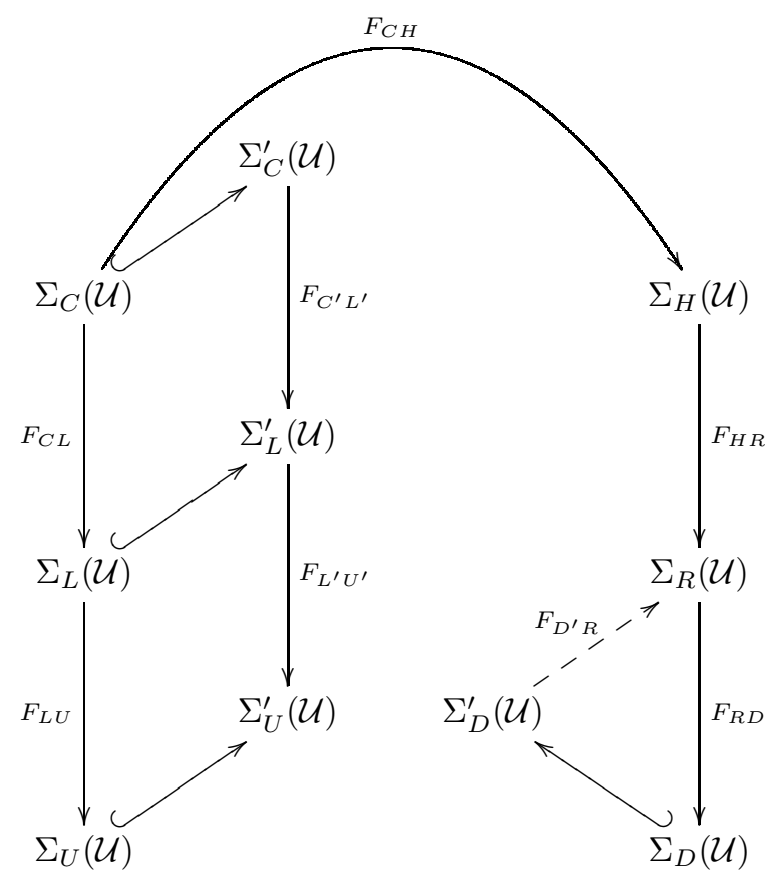

in the category of sets, where full arrows with straight tails are bijections, full arrows with curled tails are one-to-one maps while dashed arrows denote surjective maps. 
The inverse maps are obtained by transposing the labels of the map: for example, $F_{L U}^{-1}=F_{U L}$.

\section{The CURVATURE OF A DISCRETE CONNECTION}

The curvature of a connection on a bundle has many important properties that relate both to the geometry as well as the topology of the underlying space. In this section we introduce a notion of curvature for a discrete connection on a principal bundle. We motivate our definition in the fact that continuous curvatures can be seen as obstructions.

As it was mentioned in Section 2.1.1, a connection $\mathcal{A}$ on the principal $G$-bundle $\pi: Q \rightarrow Q / G$ can be seen as a certain vector bundle map that is a morphism of Lie algebroids if and only if its curvature $\mathcal{B}$ is trivial (see Appendix A of [Mac87] and Remark 5.21 below). In what follows, we introduce a notion of curvature associated to a discrete connection $\mathcal{A}_{d}$. This object is constructed as the obstruction to $\mathcal{A}_{d}: Q \times Q \rightarrow G$ being a morphism of Lie groupoids, a notion that we review next. Here we follow the notation that is customary in Geometric Mechanics (see, for instance, [MMdDM06]), that is, in some sense, opposed to the one used in Mackenzie's [Mac05].

Definition 4.1. A groupoid over a set $M$ is a set $G$ together with the following maps.

(1) The source and the target maps $\alpha, \beta: G \rightarrow M$. They define the fiber product $G_{2}:=G_{\beta} \times_{\alpha} G=\left\{\left(g_{1}, g_{2}\right) \in G \times G: \beta\left(g_{1}\right)=\alpha\left(g_{2}\right)\right\}$.

(2) A multiplication map $m: G_{2} \rightarrow G$ (usually denoted by juxtaposition) such that $\alpha\left(g_{1} g_{2}\right)=\alpha\left(g_{1}\right)$ and $\beta\left(g_{1} g_{2}\right)=\beta\left(g_{2}\right)$. It is also assumed that $g_{1}\left(g_{2} g_{3}\right)=\left(g_{1} g_{2}\right) g_{3}$ (that is, $m$ is associative, whenever it is defined).

(3) An identity section $\epsilon: M \rightarrow G$ such that, $\alpha \circ \epsilon=i d_{M}=\beta \circ \epsilon$ and so that, for all $g \in G, \epsilon(\alpha(g)) g=g$ and $g \epsilon(\beta(g))=g$.

(4) An inversion map $i: G \rightarrow G$ (denoted simply by $i(g):=g^{-1}$ ) such that $g^{-1} g=\epsilon(\beta(g))$ and $g g^{-1}=\epsilon(\alpha(g))$.

Such a groupoid is usually denoted by $G \rightrightarrows M$. A groupoid $G \rightrightarrows M$ is a Lie groupoid when $G$ and $M$ are smooth manifolds, $\alpha$ and $\beta$ are smooth submersions, and the other structure maps, $m, \epsilon$ and $i$, are smooth. A Lie groupoid is totally intransitive if $\alpha=\beta$, and it is locally trivial if $(\alpha, \beta): G \rightarrow M \times M$ - sometimes called the anchor of $G$ - is a surjective submersion.

Example 4.2. Let $M$ be a manifold. The product $M \times M$ carries a natural structure of Lie groupoid over $M$ with $\alpha:=p_{1}, \beta:=p_{2}$, so that $(M \times M)_{2}=$ $\left\{\left(\left(m_{0}, m_{1}\right),\left(m_{1}^{\prime}, m_{2}\right)\right) \in(M \times M) \times(M \times M): m_{1}=m_{1}^{\prime}\right\}=\left\{\left(\left(m_{0}, m_{1}\right),\left(m_{1}, m_{2}\right)\right):\right.$ $\left.m_{0}, m_{1}, m_{2} \in M\right\}$ and

$m\left(\left(m_{0}, m_{1}\right),\left(m_{1}, m_{2}\right)\right):=\left(m_{0}, m_{2}\right), \quad \epsilon\left(m_{0}\right):=\left(m_{0}, m_{0}\right), \quad$ and $\quad i\left(m_{0}, m_{1}\right):=\left(m_{1}, m_{0}\right)$.

This groupoid is known as the pair groupoid over $M$.

Example 4.3. Let $G$ be a Lie group. Then, $G$ can be seen as a Lie groupoid over a point $G \rightrightarrows\{0\}$ with the multiplication and inversion given by the corresponding group operations. The identity section is the assignment $0 \mapsto e$, the identity of $G$. The notion of Lie groupoid is a generalization of this example.

Example 4.4. Let $X$ be a manifold and define $\alpha=\beta=i d_{X}$. Then $X \rightrightarrows X$ is a Lie groupoid with $X_{2}=\Delta_{X} \subset X \times X$, so that $m(x, x):=x, \epsilon(x):=x$ and $i(x):=x$, for all $x \in X$. This groupoid is called the base groupoid and will be denoted by $X^{\dagger}$.

Definition 4.5. Given two Lie groupoids $G \rightrightarrows M$ and $G^{\prime} \rightrightarrows M^{\prime}$ a morphism of Lie groupoids is a smooth map $F: G \rightarrow G^{\prime}$ such that, for all $\left(g_{1}, g_{2}\right) \in G_{2}$, 
$\left(F\left(g_{1}\right), F\left(g_{2}\right)\right) \in\left(G^{\prime}\right)_{2}$ and $F\left(g_{1} g_{2}\right)=F\left(g_{1}\right) F\left(g_{2}\right)$. Such a morphism $F$ induces a smooth map $F_{0}: M \rightarrow M^{\prime}$ in such a way that

$$
\alpha^{\prime} \circ F=F_{0} \circ \alpha, \quad \beta^{\prime} \circ F=F_{0} \circ \beta \quad \text { and } \quad F \circ \epsilon=\epsilon^{\prime} \circ F_{0}
$$

(see point 3 of Lemma 5.22).

Lie groupoids and their morphisms form a category that we denote by LGpd. If $M$ is a manifold, the Lie groupoids over $M$ and the morphisms that induce the identity over $M$ form the subcategory $L G p d_{M}$ of $L G p d$.

In order to motivate our definition of curvature, we consider first the case of a discrete connection $\mathcal{A}_{d}$ on the principal $G$-bundle $\pi: Q \rightarrow Q / G$ that is globally defined, that is, $\mathfrak{U}=Q \times Q$. In this case, the discrete connection form $\mathcal{A}_{d}: Q \times Q \rightarrow$ $G$ is a smooth function between manifolds that, by Examples 4.2 and 4.3 happen to be Lie groupoids. It is natural, then, to ask if $\mathcal{A}_{d}$ is a morphism of groupoids.

In fact, there is a twist: rather than considering the Lie group $(G, \cdot)$, we consider the opposite group $\left(G^{o p}, *\right)$, that is a Lie group over the same manifold $G$ but with the product $g * g^{\prime}:=g^{\prime} \cdot g$. Then, we want to see if $\mathcal{A}_{d} \in \operatorname{hom}_{L G p d}\left(Q \times Q, G^{o p}\right)$.

As $\left(G^{o p}\right)_{2}=G \times G$, the condition $\left(\mathcal{A}_{d}\left(q_{0}, q_{1}\right), \mathcal{A}_{d}\left(q_{1}, q_{2}\right)\right) \in\left(G^{o p}\right)_{2}$ for all $\left(\left(q_{0}, q_{1}\right),\left(q_{1}, q_{2}\right)\right) \in(Q \times Q)_{2}$ is always satisfied. Then, for $\left(\left(q_{0}, q_{1}\right),\left(q_{1}, q_{2}\right)\right) \in$ $(Q \times Q)_{2}$ we have

$$
\begin{gathered}
\mathcal{A}_{d}\left(\left(q_{0}, q_{1}\right)\left(q_{1}, q_{2}\right)\right)=\mathcal{A}_{d}\left(q_{0}, q_{1}\right) * \mathcal{A}_{d}\left(q_{1}, q_{2}\right) \Longleftrightarrow \mathcal{A}_{d}\left(q_{0}, q_{2}\right)=\mathcal{A}_{d}\left(q_{1}, q_{2}\right) \mathcal{A}_{d}\left(q_{0}, q_{1}\right) \Longleftrightarrow \\
e=\mathcal{A}_{d}\left(q_{0}, q_{2}\right)^{-1} \mathcal{A}_{d}\left(q_{1}, q_{2}\right) \mathcal{A}_{d}\left(q_{0}, q_{1}\right) .
\end{gathered}
$$

In general, this last condition may fail and, in order to measure its failure, we introduce the discrete curvature of $\mathcal{A}_{d}$. The following definition, inspired by this analysis, is valid even when $\mathcal{A}_{d}$ is not globally defined.

Definition 4.6. Let $\mathcal{A}_{d}$ be a discrete connection with domain $\mathfrak{U}$ on the principal $G$-bundle $\pi: Q \rightarrow Q / G$. Let

$$
\mathfrak{U}^{(3)}:=\left\{\left(q_{0}, q_{1}, q_{2}\right) \in Q^{\times 3}:\left(q_{i}, q_{j}\right) \in \mathfrak{U} \text { for all } i, j=0,1,2\right\} .
$$

We define the discrete curvature of $\mathcal{A}_{d}$ as $\mathcal{B}_{d}: \mathfrak{U}^{(3)} \rightarrow G$ by

$$
\mathcal{B}_{d}\left(q_{0}, q_{1}, q_{2}\right):=\mathcal{A}_{d}\left(q_{0}, q_{2}\right)^{-1} \mathcal{A}_{d}\left(q_{1}, q_{2}\right) \mathcal{A}_{d}\left(q_{0}, q_{1}\right) .
$$

We say that $\mathcal{A}_{d}$ is flat if $\mathcal{B}_{d}=e$ on $\mathfrak{U}^{(3)}$.

In principle, our Definition 4.6 is not an exact parallel to the one used in the continuous setting. Still, a complete parallel will be achieved later, in Proposition 5.20 (see Remark 5.21). It also happens that this notion of discrete curvature is, among other things, the obstruction to being able to trivialize a principal bundle equipped with a discrete connection, as will be discussed elsewhere [FZ].

\section{The Discrete Atiyah Sequence in the Category of LOCAl Lie GROUPOIDS}

In Sections 2.1 and 3 we introduced and studied the discrete Atiyah sequence associated to a principal $G$-bundle $\pi: Q \rightarrow Q / G$ in the category $F b s$ of fiber bundles with a section. Motivated by the properties of the Atiyah sequence in the category of Lie algebroids, in this section we study the diagram (2.5) in the category of Lie groupoids, focusing on its relationship with the existence of discrete connections on $\pi$. In fact, as it will become evident shortly, we will need to extend the analysis to the category of local Lie groupoids, because discrete connections are usually not globally defined. 
5.1. The discrete Atiyah sequence in the category of Lie groupoids. As seen in Example 4.2, the right most object in (2.5) is a Lie groupoid. The next Example introduces such a structure for the central object.

Example 5.1. Let $\pi: Q \rightarrow Q / G$ be a principal $G$-bundle with the $G$-action $l^{Q}$. It is easy to check that $(Q \times Q) / G$ is a smooth manifold and that $\widetilde{\alpha}\left(\pi^{Q \times Q, G}\left(q_{0}, q_{1}\right)\right):=$ $\pi\left(q_{0}\right)$ and $\widetilde{\beta}\left(\pi^{Q \times Q, G}\left(q_{0}, q_{1}\right)\right):=\pi\left(q_{1}\right)$ are smooth surjective submersions. Then,

$$
\begin{aligned}
((Q \times Q) / G)_{2} & =\left\{\left(\pi^{Q \times Q, G}\left(q_{0}, q_{1}\right), \pi^{Q \times Q, G}\left(q_{1}^{\prime}, q_{2}^{\prime}\right)\right): \widetilde{\beta}\left(\pi^{Q \times Q, G}\left(q_{0}, q_{1}\right)\right)=\widetilde{\alpha}\left(\pi^{Q \times Q, G}\left(q_{1}^{\prime}, q_{2}^{\prime}\right)\right)\right\} \\
& =\left\{\left(\pi^{Q \times Q, G}\left(q_{0}, q_{1}\right), \pi^{Q \times Q, G}\left(q_{1}, q_{2}\right)\right): q_{0}, q_{1}, q_{2} \in Q\right\},
\end{aligned}
$$

and we define

$$
\begin{gathered}
\widetilde{m}\left(\left(\pi^{Q \times Q, G}\left(q_{0}, q_{1}\right), \pi^{Q \times Q, G}\left(q_{1}, q_{2}\right)\right)\right):=\pi^{Q \times Q, G}\left(q_{0}, q_{2}\right), \\
\widetilde{\epsilon}\left(\pi\left(q_{0}\right)\right):=\pi^{Q \times Q, G}\left(q_{0}, q_{0}\right), \\
\widetilde{i}\left(\pi^{Q \times Q, G}\left(q_{0}, q_{1}\right)\right):=\pi^{Q \times Q, G}\left(q_{1}, q_{0}\right)
\end{gathered}
$$

Standard computations show that all maps are well defined and smooth; furthermore, they provide a structure of Lie groupoid over $Q / G$, that is called the Atiyah or gauge groupoid associated to the principal $G$-bundle $\pi: Q \rightarrow Q / G$.

Example 5.2. The map $F_{2}$ introduced in (2.5) is a morphism of Lie groupoids, with the structures defined in Examples 5.1 and 4.2 for $(Q \times Q) / G$ and $(Q / G) \times(Q / G)$. Indeed, for $\left(\pi^{Q \times Q, G}\left(q_{0}, q_{1}\right), \pi^{Q \times Q, G}\left(q_{1}, q_{2}\right)\right) \in((Q \times Q) / G)_{2}$, we have

$$
\begin{gathered}
\left(F_{2}\left(\pi^{Q \times Q, G}\left(q_{0}, q_{1}\right)\right), F_{2}\left(\pi^{Q \times Q, G}\left(q_{1}, q_{2}\right)\right)\right)= \\
\left(\left(\pi\left(q_{0}\right), \pi\left(q_{1}\right)\right),\left(\pi\left(q_{1}\right), \pi\left(q_{2}\right)\right)\right) \\
\in((Q / G) \times(Q / G))_{2}
\end{gathered}
$$

and, then,

$$
\begin{aligned}
F_{2}\left(\pi^{Q \times Q, G}\left(q_{0}, q_{1}\right) \pi^{Q \times Q, G}\left(q_{1}, q_{2}\right)\right) & =F_{2}\left(\pi^{Q \times Q, G}\left(q_{0}, q_{2}\right)\right)=\left(\pi\left(q_{0}\right), \pi\left(q_{2}\right)\right) \\
& =\left(\pi\left(q_{0}\right), \pi\left(q_{1}\right)\right)\left(\pi\left(q_{1}\right), \pi\left(q_{2}\right)\right) \\
& =F_{2}\left(\pi^{Q \times Q, G}\left(q_{0}, q_{1}\right)\right) F_{2}\left(\pi^{Q \times Q, G}\left(q_{1}, q_{2}\right)\right) .
\end{aligned}
$$

We observe that $\left(F_{2}\right)_{0}=i d_{Q / G}$, so that $F_{2} \in \operatorname{hom}_{L G p d_{Q / G}}((Q \times Q) / G,(Q / G) \times$ $(Q / G))$.

It can be seen that, under certain conditions, when $G^{\prime} \rightrightarrows M$ is a Lie groupoid and $F: G \rightarrow G^{\prime}$ is an embedding, there is a unique Lie groupoid structure $G \rightrightarrows M$ for which $F \in \operatorname{hom}_{L G p d_{M}}\left(G, G^{\prime}\right)$. An application of this construction gives a Lie groupoid structure to the left object of (2.5) for which $F_{1}$ is a morphism of Lie groupoids.

Example 5.3. The maps $\alpha_{\widetilde{G}}, \beta_{\widetilde{G}}: \widetilde{G} \rightarrow Q / G$ defined by

$$
\alpha_{\widetilde{G}}\left(\pi^{Q \times G, G}(q, g)\right):=\pi(q) \quad \text { and } \quad \beta_{\widetilde{G}}:=\alpha_{\widetilde{G}}
$$

together with

$$
\begin{gathered}
\widetilde{G}_{2}=\left\{\left(\pi^{Q \times G, G}\left(q_{0}, g_{0}\right), \pi^{Q \times G, G}\left(q_{1}, g_{1}\right)\right): q_{0}, q_{1} \in Q, g_{0}, g_{1} \in G \text { and } \pi\left(q_{0}\right)=\pi\left(q_{1}\right)\right\} \\
=\left\{\left(\pi^{Q \times G, G}\left(q, g_{0}\right), \pi^{Q \times G, G}\left(q, g_{1}\right)\right): q \in Q, g_{0}, g_{1} \in G\right\} \\
m\left(\pi^{Q \times G, G}\left(q_{0}, g_{0}\right), \pi^{Q \times G, G}\left(l_{g}^{Q}\left(q_{0}\right), g_{1}\right)\right)=\pi^{Q \times G, G}\left(q_{0}, g_{0} g^{-1} g_{1} g\right) \\
\epsilon(\pi(q))=\pi^{Q \times G, G}(q, e) \\
i\left(\pi^{Q \times G, G}(q, g)\right)=\pi^{Q \times G, G}\left(q, g^{-1}\right) .
\end{gathered}
$$


define a Lie groupoid structure over $Q / G$ for $\widetilde{G}$. In addition, the map $F_{1}$ defined in (2.5) is a morphism of Lie groupoids for $\widetilde{G}$ with this structure and $(Q \times Q) / G$ with the structure given in Example 5.1.

Definition 5.4. Given $G_{1}, G_{3}$ in $L G p d_{M}$ such that $G_{1}$ is totally intransitive and $G_{3}$ is locally trivial, a sequence $G_{1} \stackrel{\eta_{1}}{\longrightarrow} G_{2} \stackrel{\eta_{2}}{\longrightarrow} G_{3}$ in $L G p d_{M}$ is said to be an extension of $G_{3}$ by $G_{1}$ if $\eta_{1}$ is an embedding, $\eta_{2}$ is a surjective submersion, and, for $\operatorname{Im}\left(\eta_{1}\right):=\eta_{1}\left(G_{1}\right)$ and $\operatorname{ker}\left(\eta_{2}\right):=\eta_{2}^{-1}\left(\epsilon_{G_{3}}(M)\right), \operatorname{Im}\left(\eta_{1}\right)=\operatorname{ker}\left(\eta_{2}\right)$ (as sets).

Remark 5.5. There is no completely satisfactory notion of exact sequence in the category of Lie groupoids. One version of exactness was considered by Mackenzie in [Mac87, Prop. 3.15]: it states that the sequence $G_{1} \stackrel{\eta_{1}}{\longrightarrow} G_{2} \stackrel{\eta_{2}}{\longrightarrow} G_{3}$ in $L G p d_{M}$ is exact if $\eta_{1}$ is an embedding, $\eta_{2}$ is a surjective submersion, and $\operatorname{Im}\left(\eta_{1}\right)=\operatorname{ker}\left(\eta_{2}\right)$, where $\operatorname{ker}\left(\eta_{2}\right)=\eta_{2}^{-1}\left(\epsilon_{2}(M)\right)$. Yet, in [Mac05, Defniition 1.7.15], he introduces essentially the same notion under the name extension. This is our Definition 5.4. Of course, in abelian categories, exact sequences and extensions are basically the same objects, only the perspective changes.

Proposition 5.6. The discrete Atiyah sequence (2.5) is an extension of $(Q / G) \times$ $(Q / G)$ by $\widetilde{G}$ (Definition 5.4).

Proof. It is the special case of Proposition 5.43 where $\mathcal{U}=Q \times Q$.

The natural next step is to ask if, given a discrete connection $\mathcal{A}_{d}$ with domain $\mathfrak{U}$ on the principal $G$-bundle $\pi: Q \rightarrow Q / G$, the maps $s_{L}$ and $s_{R}$ defined by (3.2) and (3.13) are morphisms of Lie groupoids. It is immediately clear that, unless $\mathcal{A}_{d}$ is globally defined, neither $s_{L}$ nor $s_{R}$ can be morphisms of groupoids (which are globally defined). Thus, we work in a more general setting, that of local Lie groupoids, that we introduce next.

5.2. Local Lie groupoids. There are several slightly different notions of local Lie groupoid. We essentially follow the one introduced in p.40 of [CDW87].

Definition 5.7. A local Lie groupoid consists of smooth manifolds $G$ and $M$ together with submersions $\alpha, \beta: G \rightarrow M$ as well as a diffeomorphism $i: G \rightarrow G$, and smooth maps $\epsilon: M \rightarrow G$ and $m: G_{m} \rightarrow G$, where $G_{m} \subset G_{2}:=G_{\beta} \times_{\alpha} G$ is an open subset, all subject to the conditions stated below. As for groupoids, $g_{1} g_{2}:=m\left(g_{1}, g_{2}\right)$ and $g^{-1}:=i(g)$.

(1) $\alpha \circ \epsilon=i d_{M}=\beta \circ \epsilon$.

(2) For all $\left(g_{1}, g_{2}\right) \in G_{m}$, we have that $\left(g_{2}^{-1}, g_{1}^{-1}\right) \in G_{m}$ and $g_{2}^{-1} g_{1}^{-1}=$ $\left(g_{1} g_{2}\right)^{-1}$.

(3) For all $g \in G,(\epsilon(\alpha(g)), g),(g, \epsilon(\beta(g))) \in G_{m}$ and $\epsilon(\alpha(g)) g=g=g \epsilon(\beta(g))$.

(4) For all $g \in G,\left(g, g^{-1}\right),\left(g^{-1}, g\right) \in G_{m}$ and $g g^{-1}=\epsilon(\alpha(g))$ and $g^{-1} g=$ $\epsilon(\beta(g))$.

(5) If $\left(g_{1}, g_{2}\right),\left(g_{2}, g_{3}\right),\left(g_{1}, g_{2} g_{3}\right) \in G_{m}$, then $\left(g_{1} g_{2}, g_{3}\right) \in G_{m}$ and $\left(g_{1} g_{2}\right) g_{3}=$ $g_{1}\left(g_{2} g_{3}\right)$.

A local Lie groupoid is totally intransitive if $\alpha=\beta$, and it is locally trivial if $(\alpha, \beta): G \rightarrow M \times M$ is a surjective submersion.

Remark 5.8. It is easy to see that a totally intransitive local Lie groupoid is a local groupoid where multiplication is only possible within each fiber and, so, each fiber is a local Lie group. This is the local version of what, in the LGpd category, are called Lie group bundles in [Mac05].

Lemma 5.9. Let $G$ be a local Lie groupoid over $M$. Then, the following assertions are true. 
(1) $\alpha, \beta: G \rightarrow M$ are onto.

(2) For $g \in G, \alpha\left(g^{-1}\right)=\beta(g)$ and $\beta\left(g^{-1}\right)=\alpha(g)$.

(3) If $\left(g_{1}, g_{2}\right) \in G_{m}$, then $\alpha\left(g_{1} g_{2}\right)=\alpha\left(g_{1}\right)$ and $\beta\left(g_{1} g_{2}\right)=\beta\left(g_{2}\right)$.

(4) $\epsilon: M \rightarrow G$ is an embedding and $\epsilon(M) \subset G$ is an embedded submanifold.

(5) $\epsilon(M)_{2}:=\left\{\left(\epsilon_{1}, \epsilon_{2}\right) \in \epsilon(M)^{2}: \beta\left(\epsilon_{1}\right)=\alpha\left(\epsilon_{2}\right)\right\} \subset G_{m}$.

Proof. Point 1 follows from condition 1 in Definition 5.7. Point 2 follows from condition 4 in Definition 5.7 and the fact that $G_{m} \subset G_{\beta} \times_{\alpha} G$. Also, point 5 follows easily using conditions 1 and 3 in Definition 5.7.

Point 3 is as follows. If $\left(g_{1}, g_{2}\right) \in G_{m}$ we have that $\left(g_{2}, g_{2}^{-1}\right) \in G_{m}$ and $\left(g_{1}, g_{2} g_{2}^{-1}\right)=\left(g_{1}, \epsilon\left(\alpha\left(g_{2}\right)\right)=\left(g_{1}, \epsilon\left(\beta\left(g_{1}\right)\right)\right) \in G_{m}\right.$. Then, by the associativity property of the local Lie groupoid, we have that $\left(g_{1} g_{2}, g_{2}^{-1}\right) \in G_{m} \subset G_{2}$, so that $\beta\left(g_{1} g_{2}\right)=\alpha\left(g_{2}^{-1}\right)=\beta\left(g_{2}\right)$ (by part 2). Similarly, it holds that $\alpha\left(g_{1} g_{2}\right)=\alpha\left(g_{1}\right)$.

Point 4 essentially follows from condition 1 in Definition 5.7. Indeed, as $\alpha \circ \epsilon=$ $i d_{M}, \epsilon$ is one-to-one and, taking derivatives at $m$, we also see that $T_{m} \epsilon$ is one-to-one. Thus, $\epsilon$ is an immersion. Also, as for $U \subset M, \epsilon(U)=\alpha^{-1}(U) \cap \epsilon(M)$, when $U$ is open, as $\alpha$ is continuous, $\alpha^{-1}(U)$ is open in $G$ and, so, $\epsilon(U)$ is open in $\epsilon(M)$ with the subspace (of $G$ ) topology. Hence, $\epsilon: M \rightarrow \epsilon(M)$ is a homeomorphism (for $\epsilon(M)$ with the subspace topology). Thus, $\epsilon$ is an embedding and $\epsilon(M)$ is an embedded submanifold of $G$.

Remark 5.10. When $G$ is a local Lie groupoid over $M$ we have $\epsilon(M)_{2}=\left\{\left(\epsilon\left(m_{1}\right), \epsilon\left(m_{2}\right)\right) \in\right.$ $G^{2}: \beta\left(\epsilon\left(m_{1}\right)\right)=\alpha\left(\epsilon\left(m_{2}\right)\right)$ for $\left.m_{1}, m_{2} \in M\right\}=\left\{(\epsilon(m), \epsilon(m)) \in G^{2}:\right.$ for $\left.m \in M\right\}$, we see that $\epsilon(M)_{2} \neq \emptyset$; then, by point 5 of Lemma $5.9, G_{m} \neq \emptyset$. Last, as $G_{m} \subset G_{2}$, this last set is not empty.

Lemma 5.11. If $G$ is a local Lie groupoid over $M$ with $G_{m}=G_{2}$, then it is a Lie groupoid over $M$.

Proof. Easy verification, using point 3 of Lemma 5.9.

Lemma 5.12. Let $G \rightrightarrows M$ be a Lie groupoid and $U \subset G$ be an open subset such that $U^{-1} \subset U$ and $\epsilon(M) \subset U$. Then $U$ is a local Lie groupoid over $M$ with the structure maps $\alpha_{U}, \beta_{U}, i_{U}$, and $\epsilon_{U}$ induced by those of $G$ by restriction or co-restriction and where the multiplication $m_{U}$ is defined as the restriction and co-restriction of the multiplication $m$ on $G$ to $U_{m}:=U_{2} \cap m^{-1}(U)$ and $U$ respectively. In particular, every Lie groupoid $G$ is a local Lie groupoid with the multiplication globally defined, that is, with $G_{m}=G_{2}$.

Proof. Being $U \subset G$ open, it is an embedded submanifold and the inclusion $j$ : $U \rightarrow G$ is a diffeomorphism onto its image. The map $\alpha_{U}:=\left.\alpha\right|_{U}$ is smooth and as $U \subset G$ is open, it is still a submersion. The same argument proves that $\beta_{U}$ is a submersion. As $i \circ i=i d_{G}$ and $i(U)=U^{-1} \subset U$, we have $U=i(i(U)) \subset i(U) \subset U$ and, then, $i(U)=U$, so that $i_{U}:=\left.i\right|_{U}$ is well defined and is, still, a diffeomorphism with $i_{U}^{2}=i d_{U}$. Notice that $m: G_{2} \rightarrow G$ is smooth and, being $U \subset G$ open, $m^{-1}(U) \subset G_{2}$ is open. Also, as $U \subset G$ is open, so is $U^{2} \subset G^{2}$; hence, $U^{2} \cap G_{2}$ is open in $G_{2}$ (which has the subspace topology in $G^{2}$ ). Then, $U_{2}=U^{2} \cap G_{2}$ and $m^{-1}(U)$ are open in $G_{2}$, therefore $U_{m}:=U_{2} \cap m^{-1}(U)$ is open in $G_{2}$ and, so, in $U_{2}$. Thus $m_{U}:=\left.m\right|_{U_{m}}: U_{m} \rightarrow U$ is well defined and, being $U_{m} \subset G_{2}$ and $U \subset G$ open, $m_{U}$ is smooth.

Next we check the compatibility conditions. As $\epsilon(M) \subset U$ we have that $\alpha_{U} \circ \epsilon_{U}=$ $\alpha \circ \epsilon=i d_{M}$ and, similarly, $\beta_{U} \circ \epsilon_{U}=i d_{M}$.

If $\left(g_{1}, g_{2}\right) \in U_{m}$, we have that $\left(g_{1}, g_{2}\right) \in G_{2}, g_{1}, g_{2} \in U$ and $g_{1} g_{2} \in U$. Then $g_{1}^{-1}, g_{2}^{-1} \in U$ and $\left(g_{2}^{-1}, g_{1}^{-1}\right) \in U_{2}$. Computing in $G, m\left(g_{2}^{-1}, g_{1}^{-1}\right)=g_{2}^{-1} g_{1}^{-1}=$ 
$\left(g_{1} g_{2}\right)^{-1} \in i(U)=U$, and we conclude that $\left(g_{2}^{-1}, g_{1}^{-1}\right) \in U_{m}$. Then as the operations are the restriction of those in the groupoid $G, i_{U}\left(g_{1} g_{2}\right)=i\left(g_{1} g_{2}\right)=\left(g_{1} g_{2}\right)^{-1}=$ $g_{2}^{-1} g_{1}^{-1}=i_{U}\left(g_{1}\right) i_{U}\left(g_{1}\right)$.

For any $g \in U$, we also have that $\epsilon_{U}\left(\alpha_{U}(g)\right)=\epsilon(\alpha(g)) \in \epsilon(M) \subset U$ and $\left(\epsilon_{U}\left(\alpha_{U}(g)\right), g\right) \in U_{2}$. In addition, as $\epsilon_{U}\left(\alpha_{U}(g)\right) g=\epsilon(\alpha(g)) g=g \in U$ we have that $\left(\epsilon_{U}\left(\alpha_{U}(g)\right), g\right) \in m^{-1}(U)$, so that $\left(\epsilon_{U}\left(\alpha_{U}(g)\right), g\right) \in U_{m}$ and, as we saw, $\epsilon_{U}\left(\alpha_{U}(g)\right) g=g$. Similarly, $\left(g, \epsilon_{U}\left(\beta_{U}(g)\right)\right) \in U_{m}$ and $g, \epsilon_{U}\left(\beta_{U}(g)\right)=g$.

For any $g \in U$, as $i_{U}(U) \subset U, g^{-1} \in U$ and, as $\alpha_{U}\left(g^{-1}\right)=\alpha\left(g^{-1}\right)=\beta(g)=$ $\beta_{U}(g)$, we see that $\left(g, g^{-1}\right) \in U_{2}$. On the other hand, as $g g^{-1}=\epsilon(\alpha(g)) \in U$, we have that $\left(g, g^{-1}\right) \in m^{-1}(U)$. Thus, $\left(g, g^{-1}\right) \in U_{m}$ and $g g^{-1}=\epsilon_{U}\left(\alpha_{U}(g)\right)$. Similarly $\left(g^{-1}, g\right) \in U_{m}$ and $g^{-1} g=\epsilon_{U}\left(\beta_{U}(g)\right)$.

If $\left(g_{1}, g_{2}\right),\left(g_{2}, g_{3}\right),\left(g_{1}, g_{2} g_{3}\right) \in U_{m}$, we have that $g_{1} g_{2} \in U, \beta_{U}\left(g_{1} g_{2}\right)=\beta\left(g_{1} g_{2}\right)=$ $\beta\left(g_{2}\right)=\beta_{U}\left(g_{2}\right)=\alpha_{U}\left(g_{3}\right)$, so that $\left(g_{1} g_{2}, g_{3}\right) \in U_{2}$. On the other hand, as $g_{1}\left(g_{2} g_{3}\right) \in$ $U$ and (operations in $G) g_{1}\left(g_{2} g_{3}\right)=\left(g_{1} g_{2}\right) g_{3}$, we have that $\left(g_{1} g_{2}, g_{3}\right) \in m^{-1}(U)$. That $g_{1}\left(g_{2} g_{3}\right)=\left(g_{1} g_{2}\right) g_{3}$ is automatic (as we saw) because $m_{U}$ is the restriction and co-restriction of $m$ to $U_{m}$ and $U$, but $m$ is known to satisfy the identity.

All together, we have verified that $U$ is a local Lie groupoid over $M$. The last assertion of the main statement follows by taking $U:=G$.

Example 5.13. Let $\pi: Q \rightarrow Q / G$ be a principal $G$-bundle; recall that, as seen in Examples 5.1 and 4.2, $(Q \times Q) / G$ and $(Q / G) \times(Q / G)$ are Lie groupoids over $Q / G$. Let $\mathcal{U} \subset Q \times Q$ be a symmetric open subset such that $\Delta_{Q} \subset \mathcal{U}$. Then $\mathcal{U}^{\prime \prime}:=$ $(\pi \times \pi)(\mathcal{U}) \subset Q / G \times Q / G$ and $\mathcal{U} / G$ are open subsets such that $i_{Q / G \times Q / G}\left(\mathcal{U}^{\prime \prime}\right)=$ $(\pi \times \pi)\left(i_{Q \times Q}(\mathcal{U})\right) \subset(\pi \times \pi)(\mathcal{U})=\mathcal{U}^{\prime \prime}$ (by the $\mathbb{Z}_{2}$-invariance of $\left.\mathcal{U}\right), i_{(Q \times Q) / G}(\mathcal{U} / G)=$ $\pi^{Q \times Q, G}\left(i_{Q \times Q}(\mathcal{U})\right) \subset \pi^{Q \times Q, G}(\mathcal{U})=\mathcal{U} / G, \epsilon_{Q / G \times Q / G}(Q / G)=\Delta_{Q / G} \subset \mathcal{U}^{\prime \prime}$ (because $\left.\Delta_{Q} \subset \mathcal{U}\right)$ and $\epsilon_{(Q \times Q) / G}(Q / G)=\pi^{Q \times Q, G}\left(\Delta_{Q}\right) \subset \pi^{Q \times Q, G}(\mathcal{U})=\mathcal{U} / G$. Then, by Lemma 5.12, $\mathcal{U}^{\prime \prime}$ and $\mathcal{U} / G$ are local Lie groupoids over $Q / G$ that are, usually, not Lie groupoids. In addition, when $\mathcal{U}$ is of $p D$-type,

$$
\begin{aligned}
\left(\mathcal{U}^{\prime \prime}\right)_{m}=\left\{\left(\left(\pi\left(q_{0}\right), \pi\left(q_{1}\right)\right),\right.\right. & \left.\left(\pi\left(q_{1}\right), \pi\left(q_{2}\right)\right)\right) \\
\left(q_{0}, q_{1}\right),\left(q_{1}, q_{2}\right) & \left.\in \mathcal{U} \text { and }\left(q_{0}, q_{2}\right) \in l_{G}^{Q \times Q_{2}}(\mathcal{U})\right\},
\end{aligned}
$$

$$
(\mathcal{U} / G)_{m}=\left\{\left(\pi^{Q \times Q, G}\left(q_{0}, q_{1}\right), \pi^{Q \times Q, G}\left(q_{1}, q_{2}\right)\right) \in((Q \times Q) / G)^{2}:\left(q_{0}, q_{1}, q_{2}\right) \in \mathcal{U}^{(3)}\right\},
$$

where $\mathcal{U}^{(3)}$ is defined as in (4.2) with $\mathcal{U}$ instead of $\mathfrak{U}$. If $\mathcal{U}$ is of $D$-type, (5.2) turns into

$$
\left(\mathcal{U}^{\prime \prime}\right)_{m}=\left\{\left(\left(\pi\left(q_{0}\right), \pi\left(q_{1}\right)\right),\left(\pi\left(q_{1}\right), \pi\left(q_{2}\right)\right)\right) \in(Q / G \times Q / G)^{2}:\left(q_{0}, q_{1}, q_{2}\right) \in \mathcal{U}^{(3)}\right\} .
$$

Remark 5.14. When $\pi: Q \rightarrow Q / G$ is a principal $G$-bundle, the manifold $\widetilde{G}$ is a Lie groupoid over $Q / G$ (Example 5.3). So, by Lemma 5.12 it is a local Lie groupoid over $Q / G$. Also, if $\mathcal{U} \subset Q \times Q$ is symmetric of $p D$-type, by Example 5.13, both $\mathcal{U}^{\prime \prime}$ and $\mathcal{U} / G$ are local Lie groupoids over $Q / G$. In what follows, whenever we consider any of these three spaces as local Lie groupoids, it will be with the structures just mentioned.

The following result is a revised version of Proposition 1.1.2 in [Mac05] adapted to the local context.

Proposition 5.15. Let $G \rightrightarrows M$ be a local Lie groupoid and $g \in G$. Then, the following assertions are true.

(1) If $h \in G$ is such that $(h, g) \in G_{m}$ and $h g=g$, then $h=\epsilon(\alpha(g))$.

(2) If $h \in G$ is such that $(g, h) \in G_{m}$ and $g h=g$, then $h=\epsilon(\beta(g))$. 
(3) If $h \in G$ is such that $(h, g) \in G_{m}$ and $h g=\epsilon(\beta(g))$, then $h=g^{-1}$.

(4) If $h \in G$ is such that $(g, h) \in G_{m}$ and $g h=\epsilon(\alpha(g))$, then $h=g^{-1}$.

Proof. (1) As $(h, g) \in G_{m} \subset G_{2}$, we have $\beta(h)=\alpha(g)$. After verifying the validity of the associativity of $m$ (using point 5 in Definition 5.7), we have $\epsilon(\alpha(g))=g g^{-1}=(h g) g^{-1}=h\left(g g^{-1}\right)=h \epsilon(\alpha(g))=h \epsilon(\beta(h))=h$. This proves point 1 , with point 2 being analogous.

3 As $(h, g) \in G_{m} \subset G_{2}$, we have $\beta(h)=\alpha(g)$. After verifying the validity of the associativity of $m$ (using point 5 in Definition 5.7 ), we have $g^{-1}=$ $\epsilon(\beta(g)) g^{-1}=(h g) g^{-1}=h\left(g g^{-1}\right)=h \epsilon(\alpha(g))=h \epsilon(\beta(h))=h$. This proves point 3 , with point 4 being analogous.

Corollary 5.16. Let $G \rightrightarrows M$ be a local Lie groupoid. Then $\epsilon(m)^{-1}=\epsilon(m)$ for each $m \in M$.

Proof. For each $m \in M$ we have $\alpha(\epsilon(m))=m$, so that by property 3 of Definition 5.7, $\epsilon(m) \epsilon(m)=\epsilon(m)$. The result follows by point 4 of Proposition 5.15.

\subsection{Morphisms of local Lie groupoids.}

Definition 5.17. Let $G \rightrightarrows M$ and $G^{\prime} \rightrightarrows M^{\prime}$ be local Lie groupoids. A smooth map $F: G \rightarrow G^{\prime}$ is a morphism of local Lie groupoids if

(1) $F\left(\epsilon_{G}(M)\right) \subset \epsilon_{G^{\prime}}\left(M^{\prime}\right)$, and

(2) for all $\left(g_{1}, g_{2}\right) \in G_{m}$, we have that $\left(F\left(g_{1}\right), F\left(g_{2}\right)\right) \in G_{m}^{\prime}$ and $F\left(g_{1} g_{2}\right)=$ $F\left(g_{1}\right) F\left(g_{2}\right)$.

A morphism of local Lie groupoids is said to be a submersion if it is a submersion as a map between smooth manifolds.

Example 5.18. Let $\pi: Q \rightarrow Q / G$ be a principal $G$-bundle. Given $\mathcal{U} \subset Q \times Q$, a symmetric $p D$-type subset, the maps $F_{1}: \widetilde{G} \rightarrow \mathcal{U} / G$ and $F_{2}: \mathcal{U} / G \rightarrow \mathcal{U}^{\prime \prime}$ are defined as the restriction and co-restriction of the homonymous maps in (2.5). Viewing both domains and codomains as local Lie groupoids (see Remark 5.14) it is easy to check by direct computation that $F_{1}$ and $F_{2}$ are morphisms of local Lie groupoids. This result also follows from Lemma 5.24, introduced later.

Next we analyze if the left and right semi-local splittings of the discrete Atiyah sequence (in the category $F b s_{Q / G}$ ) can be morphisms of local Lie groupoids.

Example 5.19. Let $\pi: Q \rightarrow Q / G$ be a connected principal $G$-bundle, $\mathcal{U} \subset Q \times Q$ be a symmetric $p D$-type subset and $s_{L}: \mathcal{U} / G \rightarrow \widetilde{G}$ a semi-local left splitting of (2.5), that is, $s_{L} \in \Sigma_{\underset{L}{G}}^{\prime}(\mathcal{U})$. As seen in Examples 5.13 and 5.3 (together with Lemma 5.11) $\mathcal{U} / G$ and $\widetilde{G}$ are local Lie groupoids over $Q / G$. We ask if $s_{L}$ is a morphism of local Lie groupoids. As $s_{L}$ is a semi-local morphism, $\phi_{\widetilde{G}} \circ s_{L}=\phi_{\mathcal{U} / G}$ and we see that, for $\left(q_{0}, q_{1}\right) \in \mathcal{U}$,

$$
\pi\left(q_{0}\right)=\phi_{\mathcal{U} / G}\left(\pi^{Q \times Q, G}\left(q_{0}, q_{1}\right)\right)=\phi_{\widetilde{G}}\left(s_{L}\left(\pi^{Q \times Q, G}\left(q_{0}, q_{1}\right)\right)\right) .
$$

Using the description of $(\mathcal{U} / G)_{m}$ provided by (5.3), for $\left(\pi^{Q \times Q, G}\left(q_{0}, q_{1}\right), \pi^{Q \times Q, G}\left(q_{1}, q_{2}\right)\right) \in$ $(\mathcal{U} / G)_{m}$, that is, satisfying $\left(q_{0}, q_{1}, q_{2}\right) \in \mathcal{U}^{(3)}$,

$$
\left(s_{L}\left(\pi^{Q \times Q, G}\left(q_{0}, q_{1}\right)\right), s_{L}\left(\pi^{Q \times Q, G}\left(q_{1}, q_{2}\right)\right)\right) \in \widetilde{G}_{m}=\widetilde{G}_{2}
$$

if and only if

$$
\beta_{\widetilde{G}}\left(s_{L}\left(\pi^{Q \times Q, G}\left(q_{0}, q_{1}\right)\right)\right)=\alpha_{\widetilde{G}}\left(s_{L}\left(\pi^{Q \times Q, G}\left(q_{1}, q_{2}\right)\right)\right) .
$$

As $\alpha_{\widetilde{G}}=\beta_{\widetilde{G}}=\phi_{\widetilde{G}}$, using (5.5), this last condition becomes

$$
\pi\left(q_{0}\right)=\phi_{\widetilde{G}}\left(s_{L}\left(\pi^{Q \times Q, G}\left(q_{0}, q_{1}\right)\right)\right)=\phi_{\widetilde{G}}\left(s_{L}\left(\pi^{Q \times Q, G}\left(q_{1}, q_{2}\right)\right)\right)=\pi\left(q_{1}\right) .
$$


Thus, equivalently, if and only if $\left(q_{0}, q_{1}\right) \in \mathcal{V}_{d}$. Hence, if $s_{L}$ is a morphism of local Lie groupoids, it should be $\mathcal{V}_{d} \supset \mathcal{U}$, thus, $\mathcal{V}_{d}=\mathcal{U}$. But then, $\mathcal{V}_{d}$ is both open and closed in the connected space $Q \times Q$, so that $\mathcal{V}_{d}=Q \times Q$. From here it is not hard to see that $\pi: Q \rightarrow Q / G$ is isomorphic to $\pi^{G, G}: G \rightarrow\{[e]\}$ for the left multiplication in $G$ action; in other words, $Q$ is a (left) torsor for $G$. Thus, in almost all cases, $s_{L}$ cannot be a morphism of local Lie groupoids.

The question of whether a semi-local right splitting $s_{R}$ of (2.5) is a morphism of local Lie groupoids is more interesting and, while we will see that it is not always the case, the obstruction is, precisely, the discrete curvature of the discrete connection $\mathcal{A}_{d}:=F_{H C}\left(F_{R H}\left(s_{R}\right)\right)$. This is explored in the next result.

Proposition 5.20. Let $\mathcal{U} \subset Q \times Q$ be a symmetric D-type subset and let $s_{R}$ : $\mathcal{U}^{\prime \prime} \rightarrow \mathcal{U} / G$ be a semi-local right splitting of (2.5) (in the category $F b s_{Q / G}$ ). Define the discrete connection $\mathcal{A}_{d}:=F_{H C}\left(F_{R H}\left(s_{R}\right)\right) \in \Sigma_{C}(\mathcal{U})$. Then $s_{R}$ is a morphism of local Lie groupoids if and only if $\mathcal{A}_{d}$ is flat.

The local Lie groupoid structures of $\mathcal{U} / G$ and $\mathcal{U}^{\prime \prime}$ implicit in the statement of Proposition 5.20 are those considered in Example 5.13.

Proof. As $s_{R}$ is a semi-local morphism in $F b s_{Q / G}$, we have $s_{R} \circ \sigma_{(Q / G) \times(Q / G)}=$ $\sigma_{(Q \times Q) / G}$ and, as $\sigma .=\epsilon$. (the Lie groupoid structure map) in the current case, we have that $s_{R}\left(\epsilon_{\mathcal{U}^{\prime \prime}}(Q / G)\right)=\epsilon_{\mathcal{U} / G}(Q / G)$.

As in the statement, define the discrete connection $\mathcal{A}_{d}:=F_{H C}\left(F_{R H}\left(s_{R}\right)\right) \in$ $\Sigma_{C}(\mathcal{U})$. Then, as $s_{R}=F_{H R}\left(F_{C H}\left(\mathcal{A}_{d}\right)\right)$, using $(2.8)$ and $(3.14)$ we have, for $\left(q_{0}, q_{1}\right) \in$ $\mathcal{U}$

$$
s_{R}\left(\pi\left(q_{0}\right), \pi\left(q_{1}\right)\right)=\pi^{Q \times Q, G}\left(q_{0}, l_{\mathcal{A}_{d}\left(q_{0}, q_{1}\right)^{-1}}^{Q}\left(q_{1}\right)\right) .
$$

As $\mathcal{U}$ is of $D$-type, recalling (5.4), all elements of $\mathcal{U}_{m}^{\prime \prime}$ are of the form

$$
\left(\left(\pi\left(q_{0}\right), \pi\left(q_{1}\right)\right),\left(\pi\left(q_{1}\right), \pi\left(q_{2}\right)\right)\right) \quad \text { with } \quad\left(q_{0}, q_{1}, q_{2}\right) \in \mathcal{U}^{(3)} .
$$

For any of them, using (5.6), we have that

$$
\begin{aligned}
& \left(s_{R}\left(\pi\left(q_{0}\right), \pi\left(q_{1}\right)\right), s_{R}\left(\pi\left(q_{1}\right), \pi\left(q_{2}\right)\right)\right) \\
& \quad=\left(\pi^{Q \times Q, G}\left(q_{0}, l_{\mathcal{A}_{d}\left(q_{0}, q_{1}\right)^{-1}}^{Q}\left(q_{1}\right)\right), \pi^{Q \times Q, G}\left(q_{1}, l_{\mathcal{A}_{d}\left(q_{1}, q_{2}\right)^{-1}}^{Q}\left(q_{2}\right)\right)\right) \\
& \quad=\left(\pi^{Q \times Q, G}\left(q_{0}, l_{\mathcal{A}_{d}\left(q_{0}, q_{1}\right)^{-1}}^{Q}\left(q_{1}\right)\right), \pi^{Q \times Q, G}\left(l_{\mathcal{A}_{d}\left(q_{0}, q_{1}\right)^{-1}}^{Q}\left(q_{1}\right), l_{\mathcal{A}_{d}\left(q_{0}, q_{1}\right)^{-1} \mathcal{A}_{d}\left(q_{1}, q_{2}\right)^{-1}}^{Q}\left(q_{2}\right)\right)\right) .
\end{aligned}
$$

It is easy to check, using (5.3), that this last expression is in $(\mathcal{U} / G)_{m}$. In this case,

$$
s_{R}\left(\left(\pi\left(q_{0}\right), \pi\left(q_{1}\right)\right)\left(\pi\left(q_{1}\right), \pi\left(q_{2}\right)\right)\right)=s_{R}\left(\pi\left(q_{0}\right), \pi\left(q_{2}\right)\right)=\pi^{Q \times Q, G}\left(q_{0}, l_{\mathcal{A}_{d}\left(q_{0}, q_{2}\right)^{-1}}^{Q}\left(q_{2}\right)\right),
$$

while, from (5.7),

$$
s_{R}\left(\pi\left(q_{0}\right), \pi\left(q_{1}\right)\right) s_{R}\left(\pi\left(q_{1}\right), \pi\left(q_{2}\right)\right)=\pi^{Q \times Q, G}\left(q_{0}, l_{\mathcal{A}_{d}\left(q_{0}, q_{1}\right)^{-1} \mathcal{A}_{d}\left(q_{1}, q_{2}\right)^{-1}}^{Q}\left(q_{2}\right)\right) .
$$

Comparing the last two expressions we have that

$$
s_{R}\left(\left(\pi\left(q_{0}\right), \pi\left(q_{1}\right)\right)\left(\pi\left(q_{1}\right), \pi\left(q_{2}\right)\right)\right)=s_{R}\left(\pi\left(q_{0}\right), \pi\left(q_{1}\right)\right) s_{R}\left(\pi\left(q_{1}\right), \pi\left(q_{2}\right)\right)
$$

if and only if $\mathcal{A}_{d}\left(q_{0}, q_{2}\right)^{-1}=\mathcal{A}_{d}\left(q_{0}, q_{1}\right)^{-1} \mathcal{A}_{d}\left(q_{1}, q_{2}\right)^{-1}$ for all $\left(q_{0}, q_{1}, q_{2}\right) \in \mathcal{U}^{(3)}$ or, recalling the definition of discrete curvature (4.3), if and only if $\mathcal{B}_{d}\left(q_{0}, q_{1}, q_{2}\right)=e$. Thus, $s_{R}$ is a morphism of local Lie groupoids if and only if $\mathcal{A}_{d}$ is flat.

Remark 5.21. A connection $\mathcal{A}$ on the principal $G$-bundle $\pi: Q \rightarrow Q / G$ can be seen as a right splitting (in the category of vector bundles) of the Atiyah sequence (2.2) (Definition on p. 188 of [Ati57] as well as Definition 4.1 in Appendix A of [Mac87]). Following Mackenzie (Definition 4.10 in Appendix A of [Mac87]), 
the curvature $\mathcal{B}$ of that connection $\mathcal{A}$ is the morphism of vector bundles $\mathcal{B}$ : $T(Q / G) \oplus T(Q / G) \rightarrow \widetilde{\mathfrak{g}}$ defined on sections by

$$
\phi_{1}\left(\mathcal{B}\left(\xi_{1}, \xi_{2}\right)\right):=\mathcal{A}\left(\left[\xi_{1}, \xi_{2}\right]\right)-\left[\mathcal{A}\left(\xi_{1}\right), \mathcal{A}\left(\xi_{2}\right)\right]
$$

for $\xi_{1}, \xi_{2} \in \mathfrak{X}(Q / G)$ and where $\phi_{1}$ is the injective morphism appearing in (2.2). Thus, $\mathcal{B}$ is the obstruction to $\mathcal{A}\left(\left[\xi_{1}, \xi_{2}\right]\right)=\left[\mathcal{A}\left(\xi_{1}\right), \mathcal{A}\left(\xi_{2}\right)\right]$ or, in other words, to $\mathcal{A}$ being a morphism of Lie algebroids, because $\mathcal{A}$ always preserves the corresponding anchor maps. The equivalence of this definition of curvature to the more traditional one is given by Proposition 4.16 in Appendix A of [Mac87]. In this respect, Proposition 5.20, shows that the curvature of a discrete connection and the curvature of a connection are both the obstructions to analogue problems in the categories of lLGpd and of Lie algebroids.

Lemma 5.22. Let $F: G \rightarrow G^{\prime}$ be a morphism of the local Lie groupoids $G \rightrightarrows M$ and $G^{\prime} \rightrightarrows M^{\prime}$. Then, the following assertions are true

(1) $F \circ \epsilon \circ \alpha=\epsilon^{\prime} \circ \alpha^{\prime} \circ F$ and $F \circ \epsilon \circ \beta=\epsilon^{\prime} \circ \beta^{\prime} \circ F$.

(2) $F\left(g^{-1}\right)=F(g)^{-1}$ for all $g \in G$.

(3) The map

$$
F_{0}: M \rightarrow M^{\prime} \quad \text { defined by } \quad F_{0}:=\alpha^{\prime} \circ F \circ \epsilon
$$

is the unique smooth map between those spaces satisfying

$$
\alpha^{\prime} \circ F=F_{0} \circ \alpha, \quad \beta^{\prime} \circ F=F_{0} \circ \beta \quad \text { and } \quad F \circ \epsilon=\epsilon^{\prime} \circ F_{0} .
$$

Proof. (1) For any $g \in G,(g, \epsilon(\beta(g))) \in G_{m}$, so that $(F(g), F(\epsilon(\beta(g)))) \in G_{m}^{\prime}$ and $F(g)=F(g \epsilon(\beta(g)))=F(g) F(\epsilon(\beta(g)))$. Applying point 2 of Proposition 5.15 to $h:=F(\epsilon(\beta(g)))$, we obtain that $F(\epsilon(\beta(g)))=\epsilon^{\prime}\left(\beta^{\prime}(F(g))\right)$. The other identity is obtained in the same way, starting from $g=\epsilon(\alpha(g)) g$.

(2) For any $g \in G,\left(g, g^{-1}\right) \in G_{m}$, so that $\left(F(g), F\left(g^{-1}\right)\right) \in G_{m}^{\prime}$ and, using point $1, F(g) F\left(g^{-1}\right)=F(\epsilon(\alpha(g)))=\epsilon^{\prime}\left(\alpha^{\prime}(F(g))\right)$. By point 4 of Proposition 5.15 applied to $h:=F\left(g^{-1}\right)$ and $F(g)$ instead of $g$, we see that $F\left(g^{-1}\right)=F(g)^{-1}$

(3) If $\widetilde{F}_{0}: M \rightarrow M^{\prime}$ satisfies $\alpha^{\prime} \circ F=\widetilde{F}_{0} \circ \alpha$, then $\widetilde{F}_{0}=\widetilde{F}_{0} \circ \alpha \circ \epsilon=\alpha^{\prime} \circ F \circ \epsilon=F_{0}$, so that $F_{0}$ is unique. Being $F_{0}$ a composition of smooth functions, it is smooth. Using point 1 of the current result, we have that $F_{0} \circ \alpha=\alpha^{\prime} \circ F \circ$ $\epsilon \circ \alpha=\alpha^{\prime} \circ \epsilon^{\prime} \circ \alpha^{\prime} \circ F=\alpha^{\prime} \circ F$ and $F_{0} \circ \beta=\alpha^{\prime} \circ F \circ \epsilon \circ \beta=\alpha^{\prime} \circ \epsilon^{\prime} \circ \beta^{\prime} \circ F=\beta^{\prime} \circ F$. Similarly, $\epsilon^{\prime} \circ F_{0}=\epsilon^{\prime} \circ \alpha^{\prime} \circ F \circ \epsilon=F \circ \epsilon \circ \alpha \circ \epsilon=F \circ \epsilon$.

Definition 5.23. All local Lie groupoids and their morphisms make a category that we denote by $l L G p d$. For a manifold $M$, if we consider only groupoids over $M$ and morphisms between those groupoids $F \in \operatorname{hom}_{l L G p d}\left(G_{1}, G_{2}\right)$ such that $F_{0}=i d_{M}$, we obtain the subcategory $l L G p d_{M}$ of $l L G p d$.

Lemma 5.24. Let $F \in \operatorname{hom}_{L G p d_{M}}\left(G, G^{\prime}\right), U \subset G$ and $U^{\prime} \subset G^{\prime}$ be open subsets such that $U^{-1} \subset U,\left(U^{\prime}\right)^{-1} \subset U^{\prime}, \epsilon_{G}(M) \subset U$ and $\epsilon_{G^{\prime}}(M) \subset U^{\prime}$. Assume that $F(U) \subset U^{\prime}$. Then $\left.F\right|_{U} ^{U^{\prime}}$ (F restricted to $U$ and co-restricted to $\left.U^{\prime}\right)$ is a morphism of local Lie groupoids, where $U$ and $U^{\prime}$ are local Lie groupoids with the structure given in Lemma 5.12. Furthermore, $\left(\left.F\right|_{U} ^{U^{\prime}}\right)_{0}=i d_{M}$.

Proof. Being $U \subset G$ and $U^{\prime} \subset G^{\prime}$ open subsets, they are embedded submanifolds and, as $F: G \rightarrow G^{\prime}$ is smooth and $F(U) \subset U^{\prime},\left.F\right|_{U} ^{U^{\prime}}: U \rightarrow U^{\prime}$ is a smooth map. Also, as $F \circ \epsilon_{G}=\epsilon_{G^{\prime}} \circ F_{0}=\epsilon_{G^{\prime}}$, it follows that $\left.F\right|_{U} ^{U^{\prime}}\left(\epsilon_{U}(M)\right)=$ $\left.F\right|_{U} ^{U^{\prime}}\left(\epsilon_{G}(M)\right)=F\left(\epsilon_{G}(M)\right) \subset \epsilon_{G^{\prime}}(M)=\epsilon_{U^{\prime}}(M)$. Next, if $\left(g_{1}, g_{2}\right) \in U_{m}=$ $U^{2} \cap m_{G}^{-1}(U) \subset G_{2}$, we have that $\left(\left.F\right|_{U} ^{U^{\prime}}\left(g_{1}\right),\left.F\right|_{U} ^{U^{\prime}}\left(g_{2}\right)\right)=\left(F\left(g_{1}\right), F\left(g_{2}\right)\right) \in\left(G^{\prime}\right)_{2}$ 
and, as $g_{1} g_{2} \in U,\left.\left.F\right|_{U} ^{U^{\prime}}\left(g_{1}\right) F\right|_{U} ^{U^{\prime}}\left(g_{2}\right)=F\left(g_{1}\right) F\left(g_{2}\right)=F\left(g_{1} g_{2}\right) \in F(U) \subset U^{\prime}$. Thus, $\left(\left.F\right|_{U} ^{U^{\prime}}\left(g_{1}\right),\left.F\right|_{U} ^{U^{\prime}}\left(g_{2}\right)\right) \in\left(U^{\prime}\right)_{m}=\left(U^{\prime}\right)^{2} \cap m_{G^{\prime}}^{-1}\left(U^{\prime}\right)$. Also, we have $\left.\left.F\right|_{U} ^{U^{\prime}}\left(g_{1}\right) F\right|_{U} ^{U^{\prime}}\left(g_{2}\right)=$ $F\left(g_{1} g_{2}\right)=\left.F\right|_{U} ^{U^{\prime}}\left(g_{1} g_{2}\right)$. All together, we have $\left.F\right|_{U} ^{U^{\prime}} \in \operatorname{hom}_{l L G p d_{M}}\left(U, U^{\prime}\right)$. The last assertion follows from $\left.F\right|_{U} ^{U^{\prime}} \circ \epsilon_{G}=F \circ \epsilon_{G}$ and (5.8).

Proposition 5.25. Let $\pi: Q \rightarrow Q / G$ be a principal $G$-bundle and $\mathcal{U} \subset Q \times Q$ be of symmetric $p D$-type. Then,

(1) for the maps $F_{1}$ and $F_{2}$ defined in Example 5.18,

$$
\widetilde{G} \stackrel{F_{1}}{\longrightarrow} \mathcal{U} / G \stackrel{F_{2}}{\longrightarrow} \mathcal{U}^{\prime \prime}
$$

is a sequence in $l L G p d_{Q / G}$ and

(2) if $\mathcal{U}$ is of D-type and $s_{R} \in \Sigma_{R}(\mathcal{U})$ is such that $\mathcal{A}_{d}:=F_{H C}\left(F_{R H}\left(s_{R}\right)\right) \in$ $\Sigma_{C}(\mathcal{U})$ is flat, then $s_{R}$ is a right splitting of (5.10) in lLGpd $/ G$.

Proof. That $\widetilde{G}, \mathcal{U} / G$ and $\mathcal{U}^{\prime \prime}$ are objects in $l L G p d_{Q / G}$ was established by Lemma 5.12 and Example 5.13. That $F_{1}$ and $F_{2}$ are morphisms in the same category follows using Lemma 5.24. On the other hand, if $s_{R}$ corresponds to a flat $\mathcal{A}_{d}$, $s_{R} \in \operatorname{hom}_{l L G p d_{Q / G}}\left(\mathcal{U}^{\prime \prime}, \mathcal{U} / G\right)$ by Proposition 5.20. Last, $F_{2} \circ s_{R}=i d_{\mathcal{U}^{\prime \prime}}$ because $s_{R}$ is a right inverse of $F_{2}$ in $F b s_{Q / G}$ by definition.

Next we establish some simple properties of totally intransitive local Lie groupoids.

Lemma 5.26. Let $M$ be a manifold. Then the base groupoid $M^{\dagger}$ defined in Example 4.4 is an initial object in $l L G p d_{M}$.

Proof. The groupoid $M^{\dagger}$ is a local Lie groupoid by Lemma 5.12. It is easy to check that if $G \in \mathrm{ob}_{l L G p d_{M}}$ the map $\epsilon_{G}: M \rightarrow G$ is in $\operatorname{hom}_{l L G p d_{M}}\left(M^{\dagger}, G\right)$. If $F \in \operatorname{hom}_{l L G p d_{M}}\left(M^{\dagger}, G\right)$ then, for each $m \in M^{\dagger}, F(m)=F(m m)=F(m) F(m)$, so that, as $\alpha_{G}\left(\epsilon_{G}(m)\right)=m$, by point 1 of Proposition 5.15 we conclude that $F(m)=\epsilon_{G}(m)$, and the statement is verified.

Example 5.27. We saw in Example 5.3 that $\widetilde{G}$ is a Lie groupoid over $Q / G$ with $\alpha_{\widetilde{G}}=\beta_{\widetilde{G}}$. The local Lie groupoid structure defined on $\widetilde{G}$ using Lemma 5.12 has the same structure maps $\alpha_{\widetilde{G}}$ and $\beta_{\widetilde{G}}$. Therefore, $\widetilde{G}$ is a totally intransitive local Lie groupoid over $Q / G$.

Lemma 5.28. Let $M$ be a manifold. Then,

(1) $M^{\dagger} \in \mathrm{ob}_{l L G p d_{M}}$ is a totally intransitive.

(2) For any $G \in \mathrm{ob}_{l L G p d_{M}}, \alpha_{G} \in \operatorname{hom}_{l L G p d_{M}}\left(G, M^{\dagger}\right)$ if and only if $G$ is totally intransitive.

(3) For any $G \in \mathrm{ob}_{l L G p d_{M}}$, either $\operatorname{hom}_{l L G p d_{M}}\left(G, M^{\dagger}\right)=\emptyset$ (G is not totally intransitive) or $\operatorname{hom}_{l L G p d_{M}}\left(G, M^{\dagger}\right)=\left\{\alpha_{G}\right\}$ (G is totally intransitive).

Proof. Point 1 follows directly from the definitions in Example 4.4. Assume now that $\alpha_{G} \in \operatorname{hom}_{l L G p d_{M}}\left(G, M^{\dagger}\right)$; as, by definition, $\left(g, \epsilon_{G}\left(\beta_{G}(g)\right)\right) \in G_{m}$ for all $g \in G$, we have that $\left(\alpha_{G}(g), \alpha_{G}\left(\epsilon_{G}\left(\beta_{G}(g)\right)\right)\right)=\left(\alpha_{G}(g), \beta_{G}(g)\right) \in M_{2}^{\dagger}=\Delta_{M}$, so that $\alpha_{G}(g)=\beta_{G}(g)$; hence, $G$ is totally intransitive. Conversely, if $G$ is totally intransitive, as $\alpha_{G} \circ \epsilon_{G}=i d_{M}=\epsilon_{M^{\dagger}}$, we have $\alpha_{G}\left(\epsilon_{G}(M)\right) \subset \epsilon_{M^{\dagger}}(M)$; also, if $\left(g_{1}, g_{2}\right) \in G_{m} \subset G_{2}$ we have that $\beta_{G}\left(g_{1}\right)=\alpha_{G}\left(g_{2}\right)$ and, as $\alpha_{G}=\beta_{G}, \alpha_{G}\left(g_{1}\right)=$ $\alpha_{G}\left(g_{2}\right)$, so that $\left(\alpha_{G}\left(g_{1}\right), \alpha_{G}\left(g_{2}\right)\right) \in \Delta_{M}=\left(M^{\dagger}\right)_{2}=\left(M^{\dagger}\right)_{m}$ and $\alpha_{G}\left(g_{1}\right) \alpha_{G}\left(g_{2}\right)=$ $\alpha_{G}\left(g_{1}\right)=\alpha_{G}\left(g_{1} g_{2}\right)$; hence, $\alpha_{G} \in \operatorname{hom}_{l L G p d_{M}}\left(G, M^{\dagger}\right)$. Hence point 2 is valid.

In order to prove point 3 , assume that $\operatorname{hom}_{l L G p d_{M}}\left(G, M^{\dagger}\right) \neq \emptyset$, so that there is $F \in \operatorname{hom}_{l L G p d_{M}}\left(G, M^{\dagger}\right)$. By point 3 of Lemma 5.22, we have that $\alpha_{G}=i d_{M} \circ \alpha_{G}=$ $\alpha_{M^{\dagger}} \circ F=i d_{M} \circ F=F$, and we conclude that, in this case, $\operatorname{hom}_{l L G p d_{M}}\left(G, M^{\dagger}\right)=$ $\left\{\alpha_{G}\right\}$. Thus, $\operatorname{hom}_{l L G p d_{M}}\left(G, M^{\dagger}\right) \neq \emptyset$ implies that $G$ is totally intransitive while 
the converse is true by point 2 of this result. All together, $\operatorname{hom}_{l L G p d_{M}}\left(G, M^{\dagger}\right) \neq \emptyset$ if and only if $G$ is totally intransitive. Negation of this last assertion concludes the proof of point 3 .

Remark 5.29. It is not hard to see using Lemma 5.28 that, if $M$ is a manifold with more than one point, then $M^{\dagger}$ is not a terminal object in $l L G p d_{M}$. Thus, because of Lemma 5.26, there are no zero objects in that category.

Next we introduce two practical results that will be used later.

Lemma 5.30. Let $\pi: Q \rightarrow Q / G$ be a principal $G$-bundle and $\mathcal{U} \subset Q \times Q$ be a symmetric $p D$-type subset. If $s \in \operatorname{hom}_{l L G p d_{Q / G}}\left(\mathcal{U}^{\prime \prime}, \mathcal{U} / G\right)$, then $s$ is a semi-local morphism between the fiber bundles with a section $\left((Q / G) \times(Q / G), \sigma_{(Q / G) \times(Q / G)}\right)$ and $\left((Q \times Q) / G, \sigma_{(Q \times Q) / G)}\right)$ (see Example 3.1).

Proof. It follows readily by noticing that the $\alpha$ and $\epsilon$ local Lie groupoid structure maps are restrictions and co-restrictions of the projection and section maps of the corresponding element of $F b s_{Q / G}$.

Lemma 5.31. Let $F \in \operatorname{hom}_{l L G p d_{M}}\left(G, G^{\prime}\right)$ such that $(F \times F)^{-1}\left(\left(G^{\prime}\right)_{m}\right) \subset G_{m}$ and that $F: G \rightarrow G^{\prime}$ is also a diffeomorphism. Then $F$ is an isomorphism in the lLGpd $d_{M}$ category.

Proof. We have to prove that $F^{-1}$, that exists and is smooth by hypothesis, is a morphism in $l L G p d_{M}$. Let $\left(g_{1}^{\prime}, g_{2}^{\prime}\right) \in\left(G^{\prime}\right)_{m}$. Then, by hypothesis, $\left(F^{-1}\left(g_{1}^{\prime}\right), F^{-1}\left(g_{2}^{\prime}\right)\right)=$ $(F \times F)^{-1}\left(g_{1}^{\prime}, g_{2}^{\prime}\right) \in G_{m}$. Also, in this case, $g_{1}^{\prime} g_{2}^{\prime}=F\left(F^{-1}\left(g_{1}^{\prime}\right)\right) F\left(F^{-1}\left(g_{2}^{\prime}\right)\right)=$ $F\left(F^{-1}\left(g_{1}^{\prime}\right) F^{-1}\left(g_{2}^{\prime}\right)\right)$, hence $F^{-1}\left(g_{1}^{\prime} g_{2}^{\prime}\right)=F^{-1}\left(g_{1}^{\prime}\right) F^{-1}\left(g_{2}^{\prime}\right)$ and $F^{-1}$ is a morphism in $l L G p d$. It is easy to see that, as $F_{0}=i d_{M},\left(F^{-1}\right)_{0}=i d_{M}$ and the statement follows.

5.4. Images, kernels and extensions. The following notions are the natural adaptation of the standard ones for Lie groupoids.

Definition 5.32. A local Lie subgroupoid of the local Lie groupoid $G \rightrightarrows M$ is a local Lie groupoid $G^{\prime} \rightrightarrows M^{\prime}$ together with $F \in \operatorname{hom}_{l L G p d}\left(G^{\prime}, G\right)$ such that $F: G^{\prime} \rightarrow G$ and the corresponding $F_{0}: M^{\prime} \rightarrow M$ are both injective immersions. A local Lie subgroupoid is embedded if both $F$ and $F_{0}$ are embeddings and it is called wide if $M^{\prime}=M$ and $F_{0}=i d_{M}$.

Proposition 5.33. Let $\eta \in \operatorname{hom}_{l L G p d_{M}}\left(G, G^{\prime}\right)$ such that $\eta: G \rightarrow G^{\prime}$ is an embedding. Then $\operatorname{Im}(\eta):=\eta(G) \subset G^{\prime}$ is a local Lie groupoid over $M$ with the structure maps induced by those of $G^{\prime}$, thus $\operatorname{Im}(\eta)$ together with the inclusion $j_{\operatorname{Im}(\eta)}: \operatorname{Im}(\eta) \rightarrow G^{\prime}$ is an embedded wide local Lie subgroupoid of $G^{\prime}$. If, in addition, $(\eta \times \eta)^{-1}\left(\left(G^{\prime}\right)_{m}\right) \subset G_{m}$, then $\left.\eta\right|^{\operatorname{Im}(\eta)} \in \operatorname{hom}_{l L G p d_{M}}(G, \operatorname{Im}(\eta))$ is an isomorphism.

Proof. In what follows, if $X \subset Y$ is a subset, we denote the corresponding inclusion map by $j^{X, Y}$. Let $I:=\operatorname{Im}(\eta) \subset G^{\prime}$ that, by hypothesis, is an embedded submanifold. Define $\alpha_{I}:=\left.\alpha^{\prime}\right|_{I}: I \rightarrow M$; as $\eta$ is a morphism, $\alpha^{\prime} \circ \eta=\alpha$ (with $\alpha$ a submersion) and, then, $T \alpha_{I}=\left.T \alpha^{\prime}\right|_{T I}$ is onto, so that $\alpha_{I}$ is a submersion. The same argument proves that $\beta_{I}:=\beta^{\prime}{ }_{I}$ is a submersion. Next we observe that, from (5.9), $\epsilon^{\prime}(M) \subset I$. We define $\epsilon_{I}:=\left.\epsilon^{\prime}\right|^{I}$, that is smooth because $\epsilon^{\prime}: M \rightarrow G^{\prime}$ is smooth and its image is contained in the embedded submanifold $I$. Let $i_{I}:=\left.i^{\prime}\right|_{I} ^{I}: I \rightarrow I$, that is well defined by point 2 of Lemma 5.22, and is a diffeomorphism because $i^{\prime}$ is a diffeomorphism of $G^{\prime}$ and $I \subset G^{\prime}$ is an embedded submanifold. Define $I_{m}:=\left(G^{\prime}\right)_{m} \cap I_{2} \subset I_{2}$; as $\left(G^{\prime}\right)_{m} \subset\left(G^{\prime}\right)_{2}=G^{\prime}{ }_{\beta^{\prime}} \times_{\alpha^{\prime}} G^{\prime}$ is an open subset and $I_{2}=I_{\beta_{I}} \times{ }_{\alpha_{I}} I \subset G^{\prime}{ }_{\beta^{\prime}} \times{ }_{\alpha^{\prime}} G^{\prime}$ has the subspace topology, we have that $I_{m} \subset I_{2}$ is an open subset. Last, define the map $m_{I}: I_{m} \rightarrow I$ by $m_{I}:=\left.m^{\prime}\right|_{I_{m}} ^{I}$; that $m_{I}$ is well defined follows from the fact that $\eta$ is a morphism, so that $m^{\prime}\left(\eta(g), \eta\left(g^{\prime}\right)\right)=\eta\left(g g^{\prime}\right) \in I$. 
Next we prove that $m_{I}$ is smooth. As $j^{I, G^{\prime}}$ is an embedding, so it $j^{I^{2},\left(G^{\prime}\right)^{2}}$ and, as $j^{I_{2},\left(G^{\prime}\right)^{2}}=j^{I^{2},\left(G^{\prime}\right)^{2}} \circ j^{I_{2}, I^{2}}$ is a composition of embeddings, it is an embedding. Then, as $j^{I_{2},\left(G^{\prime}\right)^{2}}=j^{\left(G^{\prime}\right)_{2},\left(G^{\prime}\right)^{2}} \circ j^{I_{2},\left(G^{\prime}\right)_{2}}$, we see that $j^{I_{2},\left(G^{\prime}\right)_{2}}$ is an injective immersion. Last, as $I_{2}$ and $\left(G^{\prime}\right)_{2}$ have the subspace (of $\left.\left(G^{\prime}\right)^{2}\right)$ topology, we conclude that $j^{I_{2},\left(G^{\prime}\right)_{2}}$ is an embedding. Thus, $I_{2}$ is an embedded submanifold of $\left(G^{\prime}\right)_{2}$ and, then, $I_{m}=\left(G^{\prime}\right)_{m} \cap I_{2}$ is an embedded submanifold of the open subset $\left(G^{\prime}\right)_{m} \subset\left(G^{\prime}\right)_{2}$. Consequently, as $m^{\prime}:\left(G^{\prime}\right)_{m} \rightarrow G^{\prime}$ is smooth, so is $\left.m^{\prime}\right|_{I_{m}}: I_{m} \rightarrow G^{\prime}$ and, finally, as $m^{\prime}\left(I_{m}\right) \subset I$ with $I$ embedded in $G^{\prime}, m_{I}=\left.m^{\prime}\right|_{I_{m}} ^{I}$ is a smooth map.

The verification of the five "algebraic conditions" is mechanical, mostly following from the conditions satisfied by $G^{\prime}$. Thus $\left(I, \alpha_{I}, \beta_{I}, \epsilon_{I}, i_{I}, m_{I}\right)$ is in $l L G p d_{M}$.

The last assertion in the statement follows from Lemma 5.31.

Remark 5.34. When $\eta \in \operatorname{hom}_{l L G p d_{M}}\left(G, G^{\prime}\right)$ and $G$ is a Lie groupoid, the condition $(\eta \times \eta)^{-1}\left(\left(G^{\prime}\right)_{m}\right) \subset G_{m}$ is always satisfied. Indeed, for $\left(g_{0}, g_{1}\right) \in(\eta \times \eta)^{-1}\left(\left(G^{\prime}\right)_{m}\right)$ we have that $\left(\eta\left(g_{0}\right), \eta\left(g_{1}\right)\right) \in\left(G^{\prime}\right)_{m} \subset\left(G^{\prime}\right)_{2}$, so that $\beta\left(g_{0}\right)=\beta^{\prime}\left(\eta\left(g_{0}\right)\right)=\alpha^{\prime}\left(\eta\left(g_{1}\right)\right)=$ $\alpha\left(g_{1}\right)$, thus $\left(g_{0}, g_{1}\right) \in G_{2}=G_{m}$.

Example 5.35. Let $G \rightrightarrows M$ be a local Lie groupoid. It is easy to check that the structure map $\epsilon$ is in $\operatorname{hom}_{l L G p d_{M}}\left(M^{\dagger}, G\right)$. In addition, by point 4 of Lemma 5.9, $\epsilon: M \rightarrow G$ is an embedding. Then, by Proposition 5.33, $\epsilon(M)=\operatorname{Im}(\epsilon)$ is a local Lie groupoid with the structure maps induced by those of $G$. In particular, $\epsilon(M)_{m}=G_{m} \cap \epsilon(M)^{2}=G_{m} \cap \epsilon(M)_{2}=\epsilon(M)_{2}$, so that, by Lemma 5.11, we conclude that $\epsilon(M) \rightrightarrows M$ is, in fact, a Lie groupoid over $M$. We observe that, from the proof of Proposition 5.33, $\epsilon(M)_{2}$ is an embedded submanifold of $G_{2}$.

Next we want to analyze the construction of a kernel associated to morphisms of local Lie groupoids that are surjective submersions. We start by quoting a standard result that will be used a few times in what follows.

Proposition 5.36. Let $f: X \rightarrow Y$ be a submersion and $Z \subset Y$ be an embedded submanifold such that $S:=f^{-1}(Z) \neq \emptyset$. Then $S$ is an embedded submanifold of $X$ and, for any $s \in S, T_{s} S:=\left(T_{s} f\right)^{-1}\left(T_{f(s)} Z\right)$. As a consequence, $\left.f\right|_{S} ^{Z}: S \rightarrow Z$ is a submersion.

Proof. Theorem 3.3 on p. 22 of [Hir76] and Exercise 5 on p. 32 of [GP74]. These results are stated in a more general case where $f(X)$ intersects transversely $Z$, a condition implied by the hypotheses of our statement.

Let $F \in \operatorname{hom}_{l L G p d_{M}}\left(G, G^{\prime}\right)$ and define the set

$$
\operatorname{ker}(F):=F^{-1}\left(\epsilon_{G^{\prime}}(M)\right) \subset G .
$$

For convenience, in what follows, we use $K:=\operatorname{ker}(F)$. Define the structure maps

$$
\alpha_{K}:=\left.\alpha_{G}\right|_{K}: K \rightarrow M \quad \text { and } \quad \beta_{K}:=\left.\beta_{G}\right|_{K}: K \rightarrow M .
$$

As usual, we have $K_{2}:=K_{\beta_{K}} \times_{\alpha_{K}} K \subset K^{2}$ and we define

$$
K_{m}:=K_{2} \cap G_{m} \quad \text { and } \quad m_{K}:=\left.m_{G}\right|_{K_{m}} ^{K}: K_{m} \rightarrow K .
$$

Last, we define

$$
i_{K}:=\left.i_{G}\right|_{K} ^{K}: K \rightarrow K \quad \text { and } \quad \epsilon_{K}:=\left.\epsilon_{G}\right|^{K}: M \rightarrow K
$$

Lemma 5.37. Let $F \in \operatorname{hom}_{l L G p d_{M}}\left(G, G^{\prime}\right)$ be a submersion. Then, with the notation as above, $K:=\operatorname{ker}(F)$ is an embedded submanifold of $G, \alpha_{K}=\beta_{K}$ are submersions, and $i_{K}$ and $\epsilon_{K}$ are well defined and smooth. 
Proof. Being $\epsilon_{G^{\prime}}(M) \subset G^{\prime}$ an embedded submanifold (4 in Lemma 5.9) contained in the image of the submersion $F$, by Proposition 5.36, $K:=F^{-1}\left(\epsilon_{G^{\prime}}(M)\right)$ is an embedded submanifold of $G$ and $\left.F\right|_{K} ^{\epsilon_{G^{\prime}}(M)}: K \rightarrow \epsilon_{G^{\prime}}(M)$ is a submersion. On the other hand, as $\alpha_{G^{\prime}} \circ \epsilon_{G^{\prime}}=i d_{M}$ we have that $\left.\alpha_{G^{\prime}}\right|_{\epsilon_{G^{\prime}}(M)}$ is a submersion. Thus, $\alpha_{K}=\left.\alpha_{G}\right|_{K}=\left.\left(\alpha_{G^{\prime}} \circ F\right)\right|_{K}=\left.\left.\alpha_{G^{\prime}}\right|_{\epsilon_{G^{\prime}}(M)} \circ F\right|_{K} ^{\epsilon_{G^{\prime}}(M)}$, is a submersion.

Notice that for $k \in K$ we have that $F(k)=\epsilon_{G^{\prime}}(m)$ for some $m \in M$; then, $m=$ $\alpha_{G^{\prime}}\left(\epsilon_{G^{\prime}}(m)\right)=\alpha_{G^{\prime}}(F(k))=\alpha_{G}(k)$ but, also, using $\beta_{G^{\prime}}$ instead of $\alpha_{G^{\prime}}, m=\beta_{G}(k)$. Hence, for any $k \in K, \alpha_{G}(k)=m=\beta_{G}(k)$ and we conclude that $\alpha_{K}=\beta_{K}$.

For $k \in K$, using point 2 in Lemma 5.22 and Corollary 5.16 , we have $F\left(i_{G}(k)\right)=$ $F\left(k^{-1}\right)=F(k)^{-1}=\epsilon_{G^{\prime}}\left(\alpha_{G}(k)\right)^{-1}=\epsilon_{G^{\prime}}\left(\alpha_{G}(k)\right) \in \epsilon_{G^{\prime}}(M)$. Hence $i_{G}(k) \in K$ and, then, $i_{G}(K) \subset K$, so that $i_{K}$ is well defined. Furthermore, being $i_{G}$ smooth and $K \subset G$ an embedded submanifold, $i_{K}$ is smooth.

Last, as $\epsilon_{G}: M \rightarrow G$ is smooth and $\epsilon_{G}(M) \subset K \subset G$, where the last inclusion is an embedding, we have that the co-restriction $\epsilon_{K}:=\left.\epsilon_{G}\right|^{K}$ is a smooth map.

Lemma 5.38. Let $F \in \operatorname{hom}_{l L G p d_{M}}\left(G, G^{\prime}\right)$ be a submersion. Then, with the notation as above, $K_{m} \subset K_{2}$ is an open subset and $m_{K}$ defined in (5.11) is a well defined smooth map.

Proof. As $G \in \mathrm{ob}_{l L G p d_{M}}, G_{m} \subset G_{2}$ is open and, being $G_{2} \subset G^{2}$ an embedded submanifold (hence it has the subspace topology), there is an open subset $U \subset G^{2}$ such that $G_{m}=U \cap G_{2}$. Then $K_{m}=K_{2} \cap G_{m}=K^{2} \cap G_{m}=K^{2} \cap\left(U \cap G_{2}\right)=$ $U \cap\left(K^{2} \cap G_{2}\right)=U \cap K_{2}$, proving that $K_{m} \subset K_{2}$ is open.

Now, as $F: G \rightarrow G^{\prime}$ is a submersion, so is $F \times F: G \times G \rightarrow G^{\prime} \times G^{\prime}$. Then, as $G_{2}^{\prime} \subset\left(G^{\prime}\right)^{2}$ is an embedded submanifold and as $\emptyset \neq G_{2}=(F \times F)^{-1}\left(G_{2}^{\prime}\right)$ (Remark 5.10), by Proposition 5.36, $G_{2}$ is an embedded submanifold of $G^{2}$ and $(F \times$ $F)\left.\right|_{G_{2}} ^{G_{2}^{\prime}}: G_{2} \rightarrow G_{2}^{\prime}$ is a submersion. As observed in Example 5.35, $\epsilon_{G^{\prime}}(M)_{2} \subset G_{2}^{\prime}$ is an embedded submanifold so that, by the previous argument (as $K_{2} \supset \epsilon_{G}(M)_{2} \neq \emptyset$, again by Remark 5.10), $K_{2}=\left(\left.(F \times F)\right|_{G_{2}} ^{G_{2}^{\prime}}\right)^{-1}\left(\epsilon_{G^{\prime}}(M)_{2}\right)$ is an embedded submanifold of $G_{2}$. As $G_{m} \subset G_{2}$ is open, we conclude that $K_{m}=K_{2} \cap G_{m}$ is an embedded submanifold of $G_{m}$. As $m_{G}: G_{m} \rightarrow G$ is smooth, $\left.m_{G}\right|_{K_{m}}: K_{m} \rightarrow G$ is smooth. Finally, as for $\left(k_{1}, k_{2}\right) \in K_{m}$ we have $F\left(k_{1} k_{2}\right)=F\left(k_{1}\right) F\left(k_{2}\right)=\epsilon_{G^{\prime}}\left(m_{1}\right) \epsilon_{G^{\prime}}\left(m_{2}\right)$ for some $m_{1}, m_{2} \in M$ and moreover, as $m_{2}=\alpha_{G}\left(k_{2}\right)=\beta_{G}\left(k_{1}\right)=m_{1}$, we have that, by point 3 of Definition 5.7, $F\left(k_{1} k_{2}\right)=\epsilon_{G^{\prime}}\left(m_{1}\right) \epsilon_{G^{\prime}}\left(m_{1}\right)=\epsilon_{G^{\prime}}\left(m_{1}\right)$ and we conclude that $k_{1} k_{2} \in K$, so that $m_{G}\left(K_{m}\right) \subset K$ and we see that $m_{K}:=\left.m_{G}\right|_{K_{m}} ^{K}$ is well defined. Being the restriction and co-restriction of a smooth map to embedded submanifolds, $m_{K}$ is also a smooth map.

Proposition 5.39. Let $F \in \operatorname{hom}_{l L G p d_{M}}\left(G, G^{\prime}\right)$ be a submersion. Then, with the notation as above, $K:=\operatorname{ker}(F)$ together with the structure maps $\alpha_{K}, \beta_{K}, m_{K}, i_{K}, \epsilon_{K}$ is a totally intransitive local Lie groupoid over $M$. Furthermore, the inclusion $j_{K}: K \rightarrow G$ is in $\operatorname{hom}_{l L G p d_{M}}(K, G)$.

Proof. That the structure maps are well defined and smooth as well as $\alpha_{K}=\beta_{K}$ is proved by Lemmas 5.37 and 5.38. It is easy to verify that, mostly by the structure maps being restrictions and co-restrictions of structure maps, that properties 1 through 5 in Definition 5.7 are satisfied. As $K \subset G$ is a submanifold, $j_{K}$ is smooth. Also, as $\epsilon_{K}(M)=\epsilon_{G}(M)$, we have $j_{K}\left(\epsilon_{K}(M)\right)=\epsilon_{G}(M)$. Finally, if $\left(k_{1}, k_{2}\right) \epsilon$ $K_{m} \subset G_{m}$, we have that $\left(j_{K}\left(k_{1}\right), j_{K}\left(k_{2}\right)\right)=\left(k_{1}, k_{2}\right) \in G_{m}$ and $j_{K}\left(k_{1} k_{2}\right)=k_{1} k_{2}=$ $j_{K}\left(k_{1}\right) j_{K}\left(k_{2}\right)$ because $m_{K}$ is a restriction of $m_{G}$. All together, we conclude that $j_{K} \in \operatorname{hom}_{l L G p d_{M}}(K, G)$.

Example 5.40. Let $\mathcal{U} \subset Q \times Q$ be a symmetric $p D$-type subset, so that $F_{2}$ defined in Example 5.18 is in $\operatorname{hom}_{l L G p d_{Q / G}}\left(\mathcal{U} / G, \mathcal{U}^{\prime \prime}\right)$ and is a surjective submersion by 
Lemma 3.3. Thus, by Proposition $5.39, K:=\operatorname{ker}\left(F_{2}\right)$ is a local Lie groupoid over $Q / G$. We want to give an explicit description of $K$. First off,

$$
\begin{aligned}
\operatorname{ker}\left(F_{2}\right) & =F_{2}^{-1}\left(\epsilon_{\mathcal{U}^{\prime \prime}}(Q / G)\right)=F_{2}^{-1}\left(\Delta_{Q / G}\right)=\left\{\pi^{Q \times Q, G}\left(q_{0}, q_{1}\right): \pi\left(q_{0}\right)=\pi\left(q_{1}\right)\right\} \\
& =\left\{\pi^{Q \times Q, G}\left(q, l_{g}^{Q}(q)\right): q \in Q \text { and } g \in G\right\}=\mathcal{V}_{d} / G .
\end{aligned}
$$

We also have that

$$
\begin{aligned}
\left(\operatorname{ker}\left(F_{2}\right)\right)_{2}=\{ & \left(\pi^{Q \times Q, G}\left(q_{0}, l_{g_{0}}^{Q}\left(q_{0}\right)\right), \pi^{Q \times Q, G}\left(q_{1}, l_{g_{1}}^{Q}\left(q_{1}\right)\right) \in(\mathcal{U} / G)^{2}:\right. \\
& \left.q_{0}, q_{1} \in Q, \quad g_{0}, g_{1} \in G \text { and } \pi\left(q_{0}\right)=\pi\left(q_{1}\right)\right\} \\
=\{( & \left(\pi^{Q \times Q, G}\left(q_{0}, l_{g_{0}}^{Q}\left(q_{0}\right)\right), \pi^{Q \times Q, G}\left(q_{0}, l_{g_{1}}^{Q}\left(q_{0}\right)\right) \in(\mathcal{U} / G)^{2}:\right. \\
& \left.q_{0} \in Q \text { and } g_{0}, g_{1} \in G\right\}
\end{aligned}
$$

while, from (5.3),

$$
(\mathcal{U} / G)_{m}=\left\{\left(\pi^{Q \times Q, G}\left(q_{0}, q_{1}\right), \pi^{Q \times Q, G}\left(q_{1}, q_{2}\right)\right) \in((Q \times Q) / G)^{2}:\left(q_{0}, q_{1}, q_{2}\right) \in \mathcal{U}^{(3)}\right\}
$$

so that, being $\mathcal{U}$ of $p D$-type, we have $\left(\operatorname{ker}\left(F_{2}\right)\right)_{2} \subset(\mathcal{U} / G)_{m}$. Consequently, by (5.11), $\operatorname{ker}\left(F_{2}\right)_{m}=\operatorname{ker}\left(F_{2}\right)_{2} \cap(\mathcal{U} / G)_{m}=\operatorname{ker}\left(F_{2}\right)_{2}$ and $\operatorname{ker}\left(F_{2}\right)$ is a Lie groupoid over $Q / G$. We can rephrase this analysis by saying that $\operatorname{ker}\left(F_{2}\right)=\mathcal{V}_{d} / G$ as Lie groupoids with the structure maps coming from $(Q \times Q) / G$ and that $j_{K}=i_{\mathcal{V}_{d} / G}$ is the inclusion $\mathcal{V}_{d} / G \subset(Q \times Q) / G$.

Next we introduce in $l L G p d_{M}$ the analogue of the notion of extension in the category of Lie groupoids (Definition 5.4).

Definition 5.41. Given $G_{1}, G_{3} \in \mathrm{ob}_{l L G p d_{M}}$ such that $G_{1}$ is totally intransitive and $G_{3}$ is locally trivial, a sequence $G_{1} \stackrel{\eta_{1}}{\longrightarrow} G_{2} \stackrel{\eta_{2}}{\longrightarrow} G_{3}$ in $l L G p d_{M}$ is said to be an extension of $G_{3}$ by $G_{1}$ if $\eta_{1}$ is an embedding such that $\left(\eta_{1} \times \eta_{1}\right)^{-1}\left(\left(G_{2}\right)_{m}\right) \subset\left(G_{1}\right)_{m}$, $\eta_{2}$ is a surjective submersion, and $\operatorname{Im}\left(\eta_{1}\right)=\operatorname{ker}\left(\eta_{2}\right)$ (as sets). An extension as above is said to be right split if $\eta_{2}$ has a right inverse and left split if $\eta_{1}$ has a left inverse.

Remark 5.42. Given a sequence $G_{1} \stackrel{\eta_{1}}{\longrightarrow} G_{2} \stackrel{\eta_{2}}{\longrightarrow} G_{3}$ in $l L G p d_{M}$ where $\eta_{1}$ is an embedding and $\eta_{2}$ is a surjective submersion, Propositions 5.33 and $5.39 \operatorname{turn} \operatorname{Im}\left(\eta_{1}\right)$ and $\operatorname{ker}\left(\eta_{2}\right)$ (together with the corresponding inclusions into $G_{2}$ ) into wide embedded local Lie subgroupoids of $G_{2}$ over $M$. Comparing the corresponding structures, we see that if $\operatorname{Im}\left(\eta_{1}\right)=\operatorname{ker}\left(\eta_{2}\right)$ as sets, then they are the same in $l L G p d_{M}$.

Proposition 5.43. Let $\pi: Q \rightarrow Q / G$ be a principal $G$-bundle and $\mathcal{U} \subset Q \times Q$ be of symmetric pD-type. Then, the discrete Atiyah sequence on $\mathcal{U}(5.10)$ is an extension in the $l L G p d_{Q / G}$ category.

Proof. Proposition 5.25 proves that (5.10) is a sequence in $l L G p d_{Q / G}$. As $\alpha_{\widetilde{G}}=\beta_{\widetilde{G}}$, we have that $\widetilde{G}$ is totally intransitive. Also, as $\mathcal{U}^{\prime \prime} \subset(Q / G) \times(Q / G)$ is open and the Lie groupoid $(Q / G) \times(Q / G)$ is locally trivial, so is $\mathcal{U}^{\prime \prime}$. The same idea proves that $F_{2}=\left.\left(\alpha_{(Q \times Q) / G}, \beta_{(Q \times Q) / G}\right)\right|_{\mathcal{U} / G}$ is a surjective submersion. It was established in Example 3.6 that $F_{1}$ is an embedding into $(Q \times Q) / G$ but, as $\mathcal{U} / G \subset(Q \times Q) / G$ is an open subset containing $\operatorname{Im}\left(F_{1}\right)$, we have that $F_{1}$ is an embedding as a map into $\mathcal{U} / G$. Condition $\left(F_{1} \times F_{1}\right)^{-1}\left((\mathcal{U} / G)_{m}\right) \subset(\widetilde{G})_{m}$ holds, as observed in Remark 5.34, because $\widetilde{G}$ is a Lie groupoid. Finally, as $\operatorname{Im}\left(F_{1}\right)=\mathcal{V}_{d} / G$, the statement follows from (5.12).

Proposition 5.44. Let $\pi: Q \rightarrow Q / G$ be a principal $G$-bundle and $\mathcal{U} \subset Q \times$ $Q$ be a symmetric D-type subset. Then, the bijection $F_{H R} \circ F_{C H}: \Sigma_{C}(\mathcal{U}) \rightarrow$ $\Sigma_{R}(\mathcal{U})$ determines a bijection between the subset $\Sigma_{C}^{e}(\mathcal{U}) \subset \Sigma_{C}(\mathcal{U})$ of flat discrete connections and the subset $\widetilde{\Sigma_{R}}(\mathcal{U}) \subset \Sigma_{R}(\mathcal{U})$ of right splittings in the lLGpd $d_{Q / G}$ category of extension (5.10). 
Proof. That $\widetilde{\Sigma_{R}}(\mathcal{U}) \subset \Sigma_{R}(\mathcal{U})$ follows readily from Lemma 5.30. As seen in Section 3, $\Sigma_{R}(\mathcal{U})=F_{H R}\left(F_{C H}\left(\Sigma_{C}(\mathcal{U})\right)\right)$; then, it follows from part 2 of Proposition 5.25 that $\widetilde{\Sigma_{R}}(\mathcal{U})=F_{H R}\left(F_{C H}\left(\Sigma_{C}^{e}(\mathcal{U})\right)\right)$. Last, being $F_{H R} \circ F_{C H}$ a bijection, its restriction and co-restriction is a bijection between $\Sigma_{C}^{e}(\mathcal{U})$ and $\widetilde{\Sigma_{R}}(\mathcal{U})$.

Proposition 5.39 shows that all kernels of submersive morphisms are totally intransitive local Lie groupoids. The next result shows that this is the only possibility.

Proposition 5.45. Let $M$ be a manifold and $K \in \mathrm{ob}_{l L G p d_{M}}$. Then, $K$ is totally intransitive if and only if there exist $G, G^{\prime} \in \mathrm{ob}_{l L G p d_{M}}$ and a submersion $F \in$ $\operatorname{hom}_{l L G p d_{M}}\left(G, G^{\prime}\right)$ such that $K=\operatorname{ker}(F)$.

Proof. If $K=\operatorname{ker}(F)$ for some submersion $F \in \operatorname{hom}_{l L G p d_{M}}\left(G, G^{\prime}\right)$, then $K$ is totally intransitive by Proposition 5.39. Conversely, if $K$ is totally intransitive, by point 2 of Lemma 5.28, $\alpha_{K} \in \operatorname{hom}_{l L G p d_{M}}\left(K, M^{\dagger}\right)$ is a submersion and, as $\epsilon_{M^{\dagger}}(M)=M^{\dagger}=M$, we have that $\operatorname{ker}\left(\alpha_{K}\right)=\alpha_{K}^{-1}\left(\epsilon_{M^{\dagger}}(M)\right)=\alpha_{K}^{-1}(M)=K$; then, the statement follows for $G:=K, G^{\prime}:=M^{\dagger}$ and $F:=\alpha_{K}$.

Remark 5.46. Even though the notions of kernel and extension introduced above are natural adaptations to the $l L G p d_{M}$ category of standard notions in the $L G p d_{M}$ category, it is important to realize that they also have natural categorical properties in $l L G p d_{M}$. We begin by noticing that, as there is no zero object in $l L G p d_{M}$ (see Remark 5.29), the notion of categorical kernel discussed in Remark 3.7 has to be extended. In a category $\mathcal{C}$ with an initial object 0 , given $f \in \operatorname{hom}_{\mathcal{C}}\left(A_{1}, A_{2}\right)$, a triple $(K, j, h)$ is a categorical kernel in $\mathcal{C}$ if $0 \stackrel{h}{\leftarrow} K \stackrel{j}{\rightarrow} A_{1}$ is a pullback sequence in $\mathcal{C}$ of $0 \stackrel{0^{A_{2}}}{\longrightarrow} A_{2} \stackrel{f}{\leftarrow} A_{1}$, where $0^{A_{2}}$ is the (unique) initial morphism. In the $l L G p d_{M}$ category, $M^{\dagger}$ is an initial object (Lemma 5.26), so we can apply this notion of categorical kernel. Moreover, when $K \in \mathrm{ob}_{l L G p d_{M}}$ is totally intransitive, by Lemma 5.28 , there is a unique $0_{K} \in \operatorname{hom}_{l L G p d_{M}}\left(K, M^{\dagger}\right)$, so that we may say that $(K, j)$ is a categorical kernel of a morphism $\eta$, meaning that $\left(K, j, 0_{K}\right)$ is a categorical kernel of $\eta$. Then, given $G_{1}, G_{3} \in \mathrm{ob}_{l L G p d_{M}}$ with $G_{1}$ totally intransitive and $G_{3}$ locally trivial, a sequence $G_{1} \stackrel{\eta_{1}}{\longrightarrow} G_{2} \stackrel{\eta_{2}}{\longrightarrow} G_{3}$ in $l L G p d_{M}$ is said to be a categorical extension if $\eta_{2}$ is a surjective submersion and $\left(G_{1}, \eta_{1}\right)$ is a categorical kernel of $\eta_{2}$. It can be seen that this notion of categorical extension is equivalent to that of extension given in Definition 5.41. In particular, because of Proposition 5.43, the Discrete Atiyah Sequence over $\mathcal{U}(5.10)$ is a categorical extension in $l L G p d_{Q / G}$.

5.5. Semidirect product of local Lie groupoids. In Section 3.6, we saw how right splittings of (2.5) in the $F b s_{Q / G}$ category are related to isomorphisms in the same category between (2.5) and the fiber product sequence (3.22). In this Section we explore how right splittings of sequence (5.10) in the $l L G p d_{Q / G}$ category are related to a certain semidirect product sequence in the same category.

Definition 5.47. Let $G$ be a local Lie groupoid and $H$ be a totally intransitive Lie groupoid, both over $M$. A smooth external action of $G$ on $H$ is a smooth map - : $G_{\beta_{G}} \times_{\alpha_{H}} H \rightarrow H$ with the following properties (see Definition 2 in [MM10]). As usual, we denote $\bullet(g, h)$ by $g \bullet h$.

(1) $\alpha_{H}(g \bullet h)=\alpha_{G}(g)$ for all $(g, h) \in G_{\beta_{G}} \times_{\alpha_{H}} H$,

(2) $\left(g_{1} g_{2}\right) \bullet h=g_{1} \bullet\left(g_{2} \bullet h\right)$, for all $\left(g_{1}, g_{2}\right) \in G_{m}$ such that $\left(g_{2}, h\right) \in G_{\beta_{G}} \times_{\alpha_{H}} H$,

(3) $g \bullet\left(h_{1} h_{2}\right)=\left(g \bullet h_{1}\right)\left(g \bullet h_{2}\right)$, for all $\left(h_{1}, h_{2}\right) \in H_{2}$ such that $\left(g, h_{1}\right) \in$ $G_{\beta_{G}} \times{ }_{\alpha_{H}} H$

(4) $\epsilon_{G}\left(\alpha_{H}(h)\right) \bullet h=h$, for all $h \in H$. 
Lemma 5.48. Let $\bullet: G_{\beta_{G}} \times{ }_{\alpha_{H}} H \rightarrow H$ be a smooth external action of $G$ on $H$. Then,

$$
\begin{gathered}
g \bullet \epsilon_{H}\left(\beta_{G}(g)\right)=\epsilon_{H}\left(\alpha_{G}(g)\right) \quad \text { for all } \quad g \in G, \quad \text { and } \\
(g \bullet h)^{-1}=g \bullet h^{-1} \quad \text { for all } \quad(g, h) \in G_{\beta_{G}} \times_{\alpha_{H}} H .
\end{gathered}
$$

Proof. Using point 3 in Definition 5.7 and point 3 in Definition 5.47, we have

$$
g \bullet \epsilon_{H}\left(\beta_{G}(g)\right)=g \bullet\left(\epsilon_{H}\left(\beta_{G}(g)\right) \epsilon_{H}\left(\beta_{G}(g)\right)\right)=\left(g \bullet \epsilon_{H}\left(\beta_{G}(g)\right)\right)\left(g \bullet \epsilon_{H}\left(\beta_{G}(g)\right)\right) .
$$

Then, (5.13) follows from point 1 of Proposition 5.15. Similarly, when $(g, h) \in$ $G_{\beta_{G}} \times_{\alpha_{H}} H$, it follows that $\left(g \bullet h^{-1}, g \bullet h\right) \in H_{2}$ and that

$$
\begin{aligned}
\left(g \bullet h^{-1}\right)(g \bullet h) & =g \bullet\left(h^{-1} h\right)=g \bullet \epsilon_{H}\left(\beta_{H}(h)\right)=g \bullet \epsilon_{H}\left(\alpha_{H}(h)\right) \\
& =g \bullet \epsilon_{H}\left(\beta_{G}(g)\right)=\epsilon_{H}\left(\alpha_{G}(g)\right)=\epsilon_{H}\left(\alpha_{H}(g \bullet h)\right)=\epsilon_{H}\left(\beta_{H}(g \bullet h)\right) .
\end{aligned}
$$

Then, (5.14) follows by point 3 of Proposition 5.15 .

Lemma 5.49. Let $\bullet$ be a smooth external action of the local Lie groupoid $G \rightrightarrows M$ on the totally intransitive Lie groupoid $H \rightrightarrows M$. Define $P:=H_{\beta_{H}} \times_{\alpha_{G}} G$ and the maps

$$
\begin{gathered}
\alpha_{P}, \beta_{P}: P \rightarrow M, \quad \text { by } \quad \alpha_{P}(h, g):=\alpha_{G}(g) \quad \text { and } \quad \beta_{P}(h, g):=\beta_{G}(g) \\
m_{P}: P_{m} \rightarrow P \quad \text { by } \quad m_{P}\left(\left(h_{1}, g_{1}\right),\left(h_{2}, g_{2}\right)\right):=\left(h_{1}\left(g_{1} \bullet h_{2}\right), g_{1} g_{2}\right) \\
\text { where } P_{m}:=\left\{\left(\left(h_{1}, g_{1}\right),\left(h_{2}, g_{2}\right)\right) \in P_{2}:\left(g_{1}, g_{2}\right) \in G_{m}\right\}, \\
\epsilon_{P}: M \rightarrow P \quad \text { by } \quad \epsilon_{P}(m):=\left(\epsilon_{H}(m), \epsilon_{G}(m)\right), \\
i_{P}: P \rightarrow P \quad \text { by } \quad i_{P}(h, g):=\left(\left(g^{-1} \bullet h\right)^{-1}, g^{-1}\right) .
\end{gathered}
$$

Then, $\left(P, \alpha_{P}, \beta_{P}, m_{P}, \epsilon_{P}, i_{P}\right)$ is a local Lie groupoid over $M$.

Proof. It is straightforward but lengthy, checking that the structure maps are well defined and that all conditions appearing in Definition 5.7 are met.

Notice that the smooth external action • can be fully recovered from the local multiplication $m_{P}$ of $P$.

Definition 5.50. Given a smooth external action $\bullet$ of the local Lie groupoid $G \rightrightarrows$ $M$ on the totally intransitive Lie groupoid $H \rightrightarrows M$ the local Lie groupoid $P$ defined in Lemma 5.49 is called the semidirect product local Lie groupoid defined by $\bullet$ and is denoted by $H \rtimes G \rightrightarrows M$.

Proposition 5.51. Let $\bullet$ be a smooth external action of the local Lie groupoid $G \rightrightarrows M$ on the totally intransitive Lie groupoid $H \rightrightarrows M$. Then, the diagram

$$
H \stackrel{j_{\rtimes}}{\longrightarrow} H \rtimes G \stackrel{\rho_{\rtimes}}{\longrightarrow} G
$$

is an extension of $G$ by $H$ where $H \rtimes G$ is the semidirect product of $H$ by $G$ associated to $\bullet(L e m m a ~ 5.49), j_{\rtimes}(h):=\left(h, \epsilon_{G}\left(\beta_{H}(h)\right)\right)$ and $\rho_{\rtimes}(h, g):=g$. In addition, $\rho_{\rtimes}$ is a submersion and $(H \rtimes G)_{m}=\left(\rho_{\rtimes} \times \rho_{\rtimes}\right)^{-1}\left(G_{m}\right)$. Furthermore, $s_{\rtimes}: G \rightarrow H \rtimes G$ defined by $s_{\rtimes}(g):=\left(\epsilon_{H}\left(\alpha_{G}(g)\right), g\right)$ is a splitting of the semidirect product extension.

The proof of Proposition 5.51 will follow after some preliminary work.

Lemma 5.52. With the same hypotheses of Proposition 5.51, the maps $j_{\rtimes}, \rho_{\rtimes}$ and $s_{\rtimes}$ are morphisms in the $l L G p d_{M}$ category, $j_{\rtimes}$ is an embedding and $\rho_{\rtimes}$ is a submersion. 
Proof. We only check that $j_{\rtimes} \in \operatorname{hom}_{l L G p d_{M}}(H, H \rtimes G)$ next; the cases for $\rho_{\rtimes}$ and $s_{\rtimes}$ being similar. As $\beta_{H}(h)=\alpha_{G}\left(\epsilon_{G}\left(\beta_{H}(h)\right)\right)$, we have that $j_{\rtimes}$ is a well defined map which, being the co-restriction to $P:=H \rtimes G$ of a smooth map into $H \times G$ and whose image is contained in the embedded submanifold $P \subset H \times G$, is smooth. For $m \in$ $M$ we have $j_{\rtimes}\left(\epsilon_{H}(m)\right)=\left(\epsilon_{H}(m), \epsilon_{G}\left(\beta_{H}\left(\epsilon_{H}(m)\right)\right)\right)=\left(\epsilon_{H}(m), \epsilon_{G}(m)\right)=\epsilon_{P}(m)$, so that $j_{\rtimes}\left(\epsilon_{H}(M)\right) \subset \epsilon_{P}(M)$. If $\left(h_{1}, h_{2}\right) \in H_{m}=H_{2}$, we have that $\beta_{H}\left(h_{1}\right)=$ $\alpha_{H}\left(h_{2}\right)=\beta_{H}\left(h_{2}\right)$. Then $\left(j_{\rtimes}\left(h_{1}\right), j_{\rtimes}\left(h_{2}\right)\right)=\left(\left(h_{1}, \epsilon_{G}\left(\beta_{H}\left(h_{1}\right)\right)\right),\left(h_{2}, \epsilon_{G}\left(\beta_{H}\left(h_{2}\right)\right)\right)\right) \in$ $P_{m}$ because

$$
\left(\epsilon_{G}\left(\beta_{H}\left(h_{1}\right)\right), \epsilon_{G}\left(\beta_{H}\left(h_{2}\right)\right)\right)=\left(\epsilon_{G}\left(\beta_{H}\left(h_{1}\right)\right), \epsilon_{G}\left(\beta_{H}\left(h_{1}\right)\right)\right) \in G_{m}
$$

In this case

$$
\begin{aligned}
j_{\rtimes}\left(h_{1} h_{2}\right) & =\left(h_{1} h_{2}, \epsilon_{G}\left(\beta_{H}\left(h_{1} h_{2}\right)\right)\right)=\left(h_{1} h_{2}, \epsilon_{G}\left(\beta_{H}\left(h_{2}\right)\right)\right) \\
& =\left(h_{1}\left(\epsilon_{G}\left(\alpha_{H}\left(h_{2}\right)\right) \bullet h_{2}\right), \epsilon_{G}\left(\beta_{H}\left(h_{2}\right)\right) \epsilon_{G}\left(\beta_{H}\left(h_{2}\right)\right)\right) \\
& =\left(h_{1}\left(\epsilon_{G}\left(\beta_{H}\left(h_{1}\right)\right) \bullet h_{2}\right), \epsilon_{G}\left(\beta_{H}\left(h_{1}\right)\right) \epsilon_{G}\left(\beta_{H}\left(h_{2}\right)\right)\right) \\
& =\left(h_{1}, \epsilon_{G}\left(\beta_{H}\left(h_{1}\right)\right)\right)\left(h_{2}, \epsilon_{G}\left(\beta_{H}\left(h_{2}\right)\right)\right)=j_{\rtimes}\left(h_{1}\right) j_{\rtimes}\left(h_{2}\right),
\end{aligned}
$$

and, so, $j_{\rtimes} \in \operatorname{hom}_{l L G p d}(H, P)$. A direct computation shows that $\left(j_{\rtimes}\right)_{0}=i d_{M}$, thus $j_{\rtimes} \in \operatorname{hom}_{l L G p d_{M}}(H, P)$.

Notice that if $p_{1}^{r}: H \rtimes G \rightarrow H$ is the (restriction of the) projection onto the first factor, it is a smooth map and $p_{1}^{r} \circ j_{\rtimes}=i d_{H}$. Then, by Lemma $3.21, j_{\rtimes}$ is an embedding.

Next we check that $\rho_{\rtimes}$ is a submersion. As $P=\left(\beta_{H} \times \alpha_{G}\right)^{-1}\left(\Delta_{M}\right)$ and $\beta_{H} \times \alpha_{G}$ : $H \times G \rightarrow M \times M$ is a submersion, by Proposition 5.36, for each $(h, g) \in P$, we have that $T_{(h, g)} P=\left(T_{(h, g)}\left(\beta_{H} \times \alpha_{G}\right)\right)^{-1}\left(T_{(m, m)} \Delta_{M}\right)$ for $m:=\beta_{H}(h)=\alpha_{G}(g)$. Take any $\delta g \in T_{g} G$ and define $\delta m:=T_{g} \alpha_{G}(\delta g) \in T_{m} M$; as $\beta_{H}$ is a submersion, there is $\delta h \in T_{h} H$ such that $T_{h} \beta_{H}(\delta h)=\delta m$. Then we observe that $T_{(h, m)}\left(\beta_{H} \times\right.$ $\left.\alpha_{G}\right)(\delta h, \delta g)=\left(T_{h} \beta_{H}(\delta h), T_{g} \alpha_{G}(\delta g)\right)=(\delta m, \delta m) \in T_{(m, m)} \Delta_{M}$, so that $(\delta h, \delta g) \in$ $T_{(h, g)} P$. Furthermore, $T_{(h, g)} \rho_{\rtimes}(\delta h, \delta g)=\delta g$; hence, $\rho_{\rtimes}$ is a submersion.

Proof (of Proposition 5.51). By Lemmas 5.49 and 5.52 the diagram (5.15) is a sequence in $l L G p d_{M}$ with $\rho_{\rtimes}$ a submersion. Notice that $\rho_{\rtimes} \circ s_{\rtimes}=i d_{G}$, which implies that $\rho_{\rtimes}$ is onto and, once we have proved that (5.15) is an extension, that it is split.

As seen in Lemma 5.52, $j_{\rtimes}$ is an embedding and $\rho_{\rtimes}$ is a submersion. Also, as $H$ is a Lie groupoid, $\left(j_{\rtimes}, j_{\rtimes}\right)^{-1}\left(\left(G_{2}\right)_{m}\right) \subset\left(G_{1}\right)_{m}$ as observed in Remark 5.34. On the other hand, as $\rho_{\rtimes} \circ j_{\rtimes}=\epsilon_{G} \circ \beta_{H}$, we have that $\operatorname{Im}\left(j_{\rtimes}\right) \subset \operatorname{ker}\left(\rho_{\rtimes}\right)$ while, if $(h, g) \in$ $\operatorname{ker}\left(\rho_{\rtimes}\right)$, we see that $g=\rho_{\rtimes}(h, g)=\epsilon_{G}\left(\alpha_{G}(g)\right)=\epsilon_{G}\left(\beta_{H}(h)\right)$ and, then, $(h, g)=$ $j_{\rtimes}(h) \in \operatorname{Im}\left(j_{\rtimes}\right)$, proving that $\operatorname{Im}\left(j_{\rtimes}\right)=\operatorname{ker}\left(\rho_{\rtimes}\right)$. Hence, the sequence (5.15) is an extension in $l L G p d_{M}$.

The only thing left to prove is that $(H \rtimes G)_{m}=\left(\rho_{\rtimes} \times \rho_{\rtimes}\right)^{-1}\left(G_{m}\right)$. We see that $\left(\left(h_{1}, g_{1}\right),\left(h_{2}, g_{2}\right)\right) \in P_{m}$ if and only if $\left(\left(h_{1}, g_{1}\right),\left(h_{2}, g_{2}\right)\right) \in P_{2}$ and $\left(g_{1}, g_{2}\right) \in G_{m}$. But, as $\left(g_{1}, g_{2}\right) \in G_{m}$ implies that $\left(\left(h_{1}, g_{1}\right),\left(h_{2}, g_{2}\right)\right) \in P_{2}$, we conclude that $P_{m}=$ $\left(\rho_{\rtimes} \times \rho_{\rtimes}\right)^{-1}\left(G_{m}\right)$.

We call sequence (5.15) the semidirect product sequence in the $l L G p d_{M}$ category.

Proposition 5.53. For a totally intransitive Lie groupoid $H$ let $H \stackrel{j}{\rightarrow} E \stackrel{\rho}{\rightarrow} G$ be an extension in $l L G p d_{M}$ that is right split by $s \in \operatorname{hom}_{l L G p d_{M}}(G, E)$ and where $\rho$ is a submersion and $E_{m}=(\rho \times \rho)^{-1}\left(G_{m}\right)$. Then

$$
\text { • : } G_{\beta_{G}} \times{ }_{\alpha_{H}} H \rightarrow H \quad \text { given by } \quad g \bullet h:=j^{-1}\left(s(g) j(h) s(g)^{-1}\right)
$$


is a smooth external action of $G$ on $H$ and there is an isomorphism $\Phi \in \operatorname{hom}_{l L G p d_{M}}(E, H \rtimes$ $G)$ such that the following diagram in $l L G p d_{M}$ is commutative.

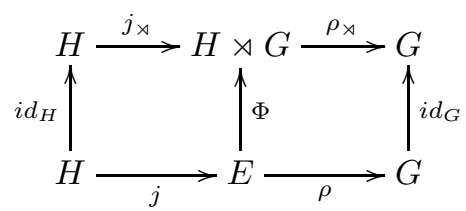

Lemma 5.54. With the hypotheses as in Proposition 5.53, • : $G_{\beta_{G}} \times_{\alpha_{H}} H \rightarrow H$ is a smooth external action.

Proof. At the level of sets, • is well defined. Indeed, for $(g, h) \in G_{\beta_{G}} \times_{\alpha_{H}} H$, we have $\beta_{G}(g)=\alpha_{H}(h)$. Then, as

$$
(\rho \times \rho)(s(g), j(h))=(\rho(s(g)), \rho(j(h)))=\left(g, \epsilon_{G}\left(\alpha_{H}(h)\right)\right)=\left(g, \epsilon_{G}\left(\beta_{G}(g)\right)\right) \in G_{m}
$$

and as, by hypothesis, $E_{m}=(\rho \times \rho)^{-1}\left(G_{m}\right)$, we see that $(s(g), j(h)) \in E_{m}$. Similarly, as

$$
\begin{aligned}
(\rho \times \rho)\left(s(g) j(h), s\left(g^{-1}\right)\right) & =\left(\rho(s(g) j(h)), \rho\left(s\left(g^{-1}\right)\right)\right)=\left(g \epsilon_{G}\left(\alpha_{H}(h)\right), g^{-1}\right) \\
& =\left(g \epsilon_{G}\left(\beta_{G}(g)\right), g^{-1}\right)=\left(g, g^{-1}\right) \in G_{m},
\end{aligned}
$$

we have that $\left(s(g) j(h), s\left(g^{-1}\right)\right) \in E_{m}$. Furthermore,

$$
\begin{aligned}
\rho\left((s(g) j(h)) s\left(g^{-1}\right)\right) & =\rho(s(g) j(h)) \rho\left(s\left(g^{-1}\right)\right)=(g \rho(j(h))) g^{-1}=\left(g \epsilon_{G}\left(\alpha_{H}(h)\right)\right) g^{-1} \\
& =\left(g \epsilon_{G}\left(\beta_{G}(g)\right)\right) g^{-1}=g g^{-1}=\epsilon_{G}\left(\alpha_{G}(g)\right),
\end{aligned}
$$

proves that $s(g) j(h) s\left(g^{-1}\right) \in \rho^{-1}\left(\epsilon_{G}(M)\right)=\operatorname{ker}(\rho)=\operatorname{Im}(j) . \quad$ As $\left.j\right|^{\operatorname{Im}(j)}: H \rightarrow$ $\operatorname{Im}(j)$ is a diffeomorphism, we see that $g \bullet h=\left(\left.j\right|^{\operatorname{Im}(j)}\right)^{-1}\left(s(g) j(h) s\left(g^{-1}\right)\right)$ is a well defined map. In addition, as $(g, h) \rightarrow s(g) j(h) s\left(g^{-1}\right)$ is a smooth map from $G_{\beta_{G}} \times{ }_{\alpha_{H}} H$ into $E$ whose image is contained in the embedded submanifold $\operatorname{Im}(j)$ and $\left(\left.j\right|^{\operatorname{Im}(j)}\right)^{-1}$ is a diffeomorphism, we conclude that $\bullet$ is a smooth map.

Recalling that $\left.j\right|^{\operatorname{Im}(j)}$ is an isomorphism in $l L G p d_{M}$, it is straightforward to check explicitly that $\bullet$ satisfies the remaining conditions to be a smooth external product.

Proof of Proposition 5.53. We know from Lemma 5.54 that $\bullet$ is a smooth external action of $G$ on $H$. Next, we want to define the map $\Phi: E \rightarrow H \rtimes G$.

For any $e \in E$, we have that

$$
\left(e, s\left(\rho\left(e^{-1}\right)\right)\right) \in E_{m} \Longleftrightarrow\left(\rho(e), \rho\left(s\left(\rho\left(e^{-1}\right)\right)\right)\right) \in G_{m}
$$

but, as $\left(\rho(e), \rho\left(s\left(\rho\left(e^{-1}\right)\right)\right)\right)=\left(\rho(e), \rho\left(e^{-1}\right)\right)=\left(\rho(e), \rho(e)^{-1}\right) \in G_{m}$, we conclude that $\left(e, s\left(\rho\left(e^{-1}\right)\right)\right) \in E_{m}$. Then, the map $\bar{\Phi}_{1}: E \rightarrow E$ defined by $\bar{\Phi}_{1}(e):=$ $e s\left(\rho\left(e^{-1}\right)\right)$ is well defined and smooth. In addition, as $\rho\left(\bar{\Phi}_{1}(e)\right)=\rho\left(e s\left(\rho\left(e^{-1}\right)\right)\right)=$ $\rho(e) \rho\left(s\left(\rho\left(e^{-1}\right)\right)\right)=\rho(e) \rho(e)^{-1}=\epsilon_{G}\left(\alpha_{G}(\rho(e))\right)=\epsilon_{G}\left(\alpha_{E}(e)\right)$, we see that the image of $\bar{\Phi}_{1}$ is contained in the embedded submanifold $\operatorname{ker}(\rho)=\operatorname{Im}(j) \subset E$, so that $\Phi_{1}: E \rightarrow H$ defined by $\Phi_{1}(e):=j_{c}{ }^{-1}\left(\Phi_{1}(e)\right)$, where $j_{c}:=\left.j\right|^{\operatorname{Im}(j)}$, is a smooth map. We define $\bar{\Phi}: E \rightarrow H \times G$ by

$$
\bar{\Phi}(e):=\left(\Phi_{1}(e), \rho(e)\right)=\left(j_{c}^{-1}\left(e s\left(\rho\left(e^{-1}\right)\right)\right), \rho(e)\right),
$$

that clearly is a smooth map. As $\beta_{H}\left(j_{c}{ }^{-1}\left(e s\left(\rho\left(e^{-1}\right)\right)\right)\right)=\beta_{K}\left(e s\left(\rho\left(e^{-1}\right)\right)\right)=$ $\beta_{E}\left(s\left(\rho\left(e^{-1}\right)\right)\right)=\beta_{G}\left(\rho\left(e^{-1}\right)\right)=\alpha_{G}(\rho(e))$, we see that the image of $\bar{\Phi}$ is contained in the embedded submanifold $H \rtimes G \subset H \times G$. Hence, its co-restriction $\Phi: E \rightarrow H \rtimes G$ defined by $\Phi:=\left.\bar{\Phi}\right|^{H \rtimes G}$ is a smooth map. 
The rest of the proof follows from Lemma 5.55, where diagram (5.17) is seen to be commutative in the $l L G p d_{M}$ category, and Lemma 5.56 , where $\Phi$ is seen to be an isomorphism in the same category.

Lemma 5.55. With the hypotheses as in Proposition 5.53 and $\Phi$ defined in the proof of that result, diagram (5.17) is commutative in the lLGpd ${ }_{M}$ category.

Proof. Let $P:=H \rtimes G$. A direct computation shows that $\Phi \circ \epsilon_{E}=\epsilon_{P}$, so that $\Phi\left(\epsilon_{E}(M)\right) \subset \epsilon_{P}(M)$.

For any $\left(e_{1}, e_{2}\right) \in E_{m}=(\rho \times \rho)^{-1}\left(G_{m}\right)$ we have that $\left(\rho\left(e_{1}\right), \rho\left(e_{2}\right)\right) \in G_{m}$. Then, as

$$
\left(\rho_{\rtimes}\left(\Phi\left(e_{1}\right)\right), \rho_{\rtimes}\left(\Phi\left(e_{2}\right)\right)\right)=\left(\rho\left(e_{1}\right), \rho\left(e_{2}\right)\right) \in G_{m},
$$

we have that $\left(\Phi\left(e_{1}\right), \Phi\left(e_{2}\right)\right) \in\left(\rho_{\rtimes} \times \rho_{\rtimes}\right)^{-1}\left(G_{m}\right)=P_{m}$ (by Proposition 5.51). In this case,

$$
\begin{aligned}
\Phi\left(e_{1} e_{2}\right) & =\left(j_{c}^{-1}\left(\left(e_{1} e_{2}\right) s\left(\rho\left(\left(e_{1} e_{2}\right)^{-1}\right)\right)\right), \rho\left(e_{1} e_{2}\right)\right)=\left(j_{c}^{-1}\left(\left(e_{1} e_{2}\right) s\left(\rho\left(e_{2}^{-1} e_{1}^{-1}\right)\right)\right), \rho\left(e_{1} e_{2}\right)\right) \\
& =\left(j_{c}^{-1}(\underbrace{e_{1} s\left(\rho\left(e_{1}^{-1}\right)\right)}_{\in \operatorname{ker}(\rho)=\operatorname{Im}(j)} \underbrace{s\left(\rho\left(e_{1}\right)\right) e_{2} s\left(\rho\left(e_{2}^{-1} e_{1}^{-1}\right)\right)}_{\in \operatorname{ker}(\rho)=\operatorname{Im}(j)}), \rho\left(e_{1} e_{2}\right)\right) \\
& =\left(j_{c}^{-1}\left(e_{1} s\left(\rho\left(e_{1}^{-1}\right)\right)\right) j_{c}^{-1}\left(s\left(\rho\left(e_{1}\right)\right) e_{2} s\left(\rho\left(e_{2}^{-1} e_{1}^{-1}\right)\right)\right), \rho\left(e_{1} e_{2}\right)\right) \\
& =\left(j_{c}^{-1}\left(e_{1} s\left(\rho\left(e_{1}^{-1}\right)\right)\right) j_{c}^{-1}\left(s\left(\rho\left(e_{1}\right)\right) e_{2} s\left(\rho\left(e_{2}^{-1}\right)\right) s\left(\rho\left(e_{1}^{-1}\right)\right)\right), \rho\left(e_{1} e_{2}\right)\right) \\
& =\left(j_{c}^{-1}\left(e_{1} s\left(\rho\left(e_{1}^{-1}\right)\right)\right) j_{c}^{-1}\left(s\left(\rho\left(e_{1}\right)\right) j\left(j_{c}^{-1}\left(e_{2} s\left(\rho\left(e_{2}^{-1}\right)\right)\right) s\left(\rho\left(e_{1}\right)^{-1}\right)\right), \rho\left(e_{1} e_{2}\right)\right)\right. \\
& =\left(j_{c}^{-1}\left(e_{1} s\left(\rho\left(e_{1}^{-1}\right)\right)\right)\left(\rho\left(e_{1}\right) \bullet j_{c}^{-1}\left(e_{2} s\left(\rho\left(e_{2}^{-1}\right)\right)\right)\right), \rho\left(e_{1}\right) \rho\left(e_{2}\right)\right) \\
& =\left(j_{c}^{-1}\left(e_{1} s\left(\rho\left(e_{1}^{-1}\right)\right)\right), \rho\left(e_{1}\right)\right)\left(j_{c}^{-1}\left(e_{2} s\left(\rho\left(e_{2}^{-1}\right)\right)\right), \rho\left(e_{2}\right)\right)=\Phi\left(e_{1}\right) \Phi\left(e_{2}\right),
\end{aligned}
$$

and we see that $\Phi \in \operatorname{hom}_{l L G p d}(E, P)$. Direct computations show that

$$
\Phi_{0}=i d_{M}, \quad \Phi \circ j=j_{\rtimes} \quad \text { and } \quad \rho_{\rtimes} \circ \Phi=\rho,
$$

so that $\Phi \in \operatorname{hom}_{l L G p d_{M}}(E, P)$ and diagram (5.17) is commutative.

Lemma 5.56. With the hypotheses of Proposition 5.53 and $\Phi$ defined in the proof of that result, $\Phi$ is an isomorphism in the $l L G p d_{M}$ category.

Proof. Let $P:=H \rtimes G$. Consider now the smooth maps $p_{1}^{r}: P \rightarrow H$ and $p_{2}^{r}: P \rightarrow G$ obtained as restrictions to $P$ of the canonical projections onto the corresponding factor. Then, the maps $f_{1}, f_{2}: P \rightarrow E$ defined by $f_{1}(p):=j_{c}\left(p_{1}^{r}(p)\right)$ and $f_{2}(p):=$ $s\left(p_{2}^{r}(p)\right)$ are smooth. Furthermore, for $(h, g) \in P$, that is, satisfying $\beta_{H}(h)=\alpha_{G}(g)$, we have that

$$
\begin{aligned}
\left(\rho\left(f_{1}(h, g)\right), \rho\left(f_{2}(h, g)\right)\right) & =\left(\rho\left(j_{c}(h)\right), \rho(s(g))\right)=\left(\epsilon_{G}\left(\alpha_{H}(h)\right), g\right) \\
& =\left(\epsilon_{G}\left(\beta_{H}(h)\right), g\right)=\left(\epsilon_{G}\left(\alpha_{G}(g)\right), g\right) \in G_{m},
\end{aligned}
$$

so that $\left(f_{1}(h, g), f_{2}(h, g)\right) \in(\rho \times \rho)^{-1}\left(G_{m}\right)=E_{m}$. In this case, the map $\Psi: P \rightarrow E$ defined by $\Psi(p):=f_{1}(p) f_{2}(p)$ is well defined and, taking into account that $m_{E}$ is smooth, we see that $\Psi$ is a smooth map.

As for any $(h, g) \in P$ we have

$$
\begin{aligned}
\Phi(\Psi(h, g)) & =\Phi\left(j_{c}(h) s(g)\right)=\left(j_{c}^{-1}\left(j_{c}(h) s(g) s\left(\rho\left(\left(j_{c}(h) s(g)\right)^{-1}\right)\right)\right), \rho\left(j_{c}(h) s(g)\right)\right) \\
& =\left(j_{c}{ }^{-1}\left(j_{c}(h) s(g) s\left(\rho\left(s(g)^{-1} j_{c}(h)^{-1}\right)\right)\right), \rho\left(j_{c}(h)\right) \rho(s(g))\right) \\
& =(j_{c}{ }^{-1}\left(j_{c}(h) s(g) s(\underbrace{\rho(s(g))}_{=g})^{-1} s(\underbrace{\rho\left(j_{c}(h)\right)}_{=\epsilon_{G}\left(\beta_{H}(h)\right)})^{-1}\right), \underbrace{\rho\left(j_{c}(h)\right)}_{\epsilon_{G}\left(\beta_{H}(h)\right)} \underbrace{\rho(s(g))}_{=g}) \\
& =\left(j_{c}^{-1}\left(j_{c}(h) \epsilon_{E}\left(\beta_{H}(h)\right)\right), \epsilon_{G}\left(\alpha_{G}(g)\right) g\right) \\
& =\left(j_{c}{ }^{-1}\left(j_{c}(h) \epsilon_{E}\left(\beta_{K}\left(j_{c}(h)\right)\right)\right), g\right)=\left(j_{c}^{-1}\left(j_{c}(h) \epsilon_{E}\left(\beta_{E}\left(j_{c}(h)\right)\right)\right), g\right) \\
& =\left(j_{c}{ }^{-1}\left(j_{c}(h)\right), g\right)=(h, g),
\end{aligned}
$$


we see that $\Phi \circ \Psi=i d_{P}$. Similarly, for any $e \in E$,

$$
\begin{aligned}
\Psi(\Phi(e)) & =\Psi\left(j_{c}^{-1}\left(e s\left(\rho\left(e^{-1}\right)\right)\right), \rho(e)\right) \\
& =j_{c}\left(j_{c}{ }^{-1}\left(e s\left(\rho\left(e^{-1}\right)\right)\right)\right) s(\rho(e))=e s\left(\rho\left(e^{-1}\right)\right) s(\rho(e))=e,
\end{aligned}
$$

so that $\Psi \circ \Phi=i d_{M}$. Thus, $\Psi=\Phi^{-1}$ and we have proved that $\Phi$ is a diffeomorphism.

Let $\left(e_{1}, e_{2}\right) \in(\Phi \times \Phi)^{-1}\left(P_{m}\right)$, so that $\left(\Phi\left(e_{1}\right), \Phi\left(e_{2}\right)\right) \in P_{m}$. As $\rho_{\rtimes}$ is a morphism in $l L G p d_{M}$, we have that $\left(\rho_{\rtimes}\left(\Phi\left(e_{1}\right)\right), \rho_{\rtimes}\left(\Phi\left(e_{2}\right)\right)\right) \in G_{m}$. But, then, by (5.18), $\left(\rho\left(e_{1}\right), \rho\left(e_{2}\right)\right) \in G_{m}$ and, as $E_{m}=(\rho \times \rho)^{-1}\left(G_{m}\right)$, we see that $\left(e_{1}, e_{2}\right) \in E_{m}$. All together, we proved that $(\Phi \rtimes \Phi)^{-1}\left(P_{m}\right) \subset E_{m}$.

All together, by Lemma 5.31, $\Phi$ is an isomorphism in the $l L G p d_{M}$ category.

Remark 5.57. A natural question is if all smooth external actions • of $G$ on $H$ come from split extensions via the construction described in Proposition 5.53. The answer is yes and it follows from the easily verified identity

$$
j_{\rtimes}(g \bullet h)=s_{\rtimes}(g) j_{\rtimes}(h) s_{\rtimes}(g)^{-1} \quad \text { for all } \quad(g, h) \in G_{\beta_{G}} \times_{\alpha_{H}} H
$$

and, then, by the construction of Proposition 5.53 applied to the semidirect product extension (5.15) with $\Phi=i d_{H \rtimes G}$ (recall that (5.15) is split by Proposition 5.51).

Let $H$ be a totally intransitive Lie groupoid and

$$
H \stackrel{j}{\rightarrow} E \stackrel{\rho}{\rightarrow} G
$$

be an extension in the $l L G p d_{M}$ category such that

$$
E_{m}=(\rho \times \rho)^{-1}\left(G_{m}\right) .
$$

Let $\widetilde{\Sigma_{I}}$ be the set of all isomorphisms $\Phi \in \operatorname{hom}_{l L G p d_{M}}(E, H \rtimes G)$ where $H \rtimes G$ is constructed using any arbitrary smooth external action of $G$ on $H$, such that diagram (5.17) is commutative. Also, we let $\widetilde{\Sigma_{R}}$ be the set of right splittings of $H \stackrel{j}{\rightarrow} E \stackrel{\rho}{\rightarrow} G$ in the $l L G p d_{M}$ category. There is a map $\widetilde{F}_{R I}: \widetilde{\Sigma_{R}} \rightarrow \widetilde{\Sigma_{I}}$ defined by $\widetilde{F}_{R I}(s):=\Phi$, the isomorphism provided by Proposition 5.53. Conversely, define $\widetilde{F}_{I R}: \widetilde{\Sigma_{I}} \rightarrow \widetilde{\Sigma_{R}}$ by $\widetilde{F}_{I R}(\Phi):=s$, for $s:=\Phi^{-1} \circ s_{\rtimes}$, where $s_{\rtimes}$ is defined in Proposition 5.51.

Theorem 5.58. With the definitions as above, both $\widetilde{F}_{R I}$ and $\widetilde{F}_{I R}$ are well defined maps that are inverses of each other. In other words, in the lLGpd $M$ category, there is a bijective correspondence between the right splittings of an extension (5.19) satisfying (5.20) and the isomorphisms with semidirect product sequences.

Proof. That $\widetilde{F}_{R I}$ is well defined is the content of Proposition 5.53. If $\Phi \in \widetilde{\Sigma_{I}}$, then it is in $\operatorname{hom}_{l L G p d_{M}}(E, H \rtimes G)$ and it is an isomorphism; thus, $\Phi^{-1} \in \operatorname{hom}_{l L G p d_{M}}(H \rtimes$ $G, E)$ and, as $s_{\rtimes} \in \operatorname{hom}_{l L G p d_{M}}(G, H \rtimes G)$ by Proposition 5.51, then $s:=\Phi^{-1} \diamond_{\rtimes} \in$ $\operatorname{hom}_{l L G p d_{M}}(G, E)$ and $\rho \circ s=\rho \circ \Phi^{-1} \circ s_{\rtimes}=\rho_{\rtimes} \circ \Phi \circ \Phi^{-1} \circ s_{\rtimes}=i d_{G}$, so that $s \in \widetilde{\Sigma_{R}}$, and $\widetilde{F}_{I R}$ is well defined.

For $s \in \widetilde{\Sigma_{R}}$, let $\Phi:=\widetilde{F}_{R I}(s)$ and $\bar{s}:=\widetilde{F}_{I R}(\Phi)$. Then, for $g \in G$, recalling the expression for $\Phi^{-1}=\Psi$ given in the proof of Lemma 5.56,

$$
\begin{aligned}
\bar{s}(g) & =\Phi^{-1}\left(s_{\rtimes}(g)\right)=\Phi^{-1}\left(\epsilon_{H}\left(\alpha_{G}(g)\right), g\right)=j_{c}\left(\epsilon_{H}\left(\alpha_{G}(g)\right)\right) s(g)=\epsilon_{K}\left(\alpha_{G}(g)\right) s(g) \\
& =\epsilon_{E}\left(\alpha_{E}(s(g))\right) s(g)=s(g),
\end{aligned}
$$

and we see that $\widetilde{F}_{I R} \circ \widetilde{F}_{R I}=i d_{\widetilde{\Sigma_{R}}}$.

For $\Phi \in \widetilde{\Sigma_{I}}$, let $s:=\widetilde{F}_{I R}(\Phi)$ and $\bar{\Phi}:=\widetilde{F}_{I R}(s)$. If $p_{j}^{r}$ is the restriction of the projection onto the corresponding component and $\widetilde{\Phi} \in \widetilde{\Sigma_{I}}$, then, by the commutativity of (5.17), $p_{2}^{r} \circ \widetilde{\Phi}=\rho_{\rtimes} \circ \widetilde{\Phi}=\rho$ and, in particular, $p_{2}^{r} \circ \Phi=\rho=p_{2}^{r} \circ \bar{\Phi}$. 
For $e \in E$, as seen in the proof of Proposition 5.53, we have

$$
\left(p_{1}^{r} \circ \bar{\Phi}\right)(e)=j_{c}^{-1}\left(e s\left(\rho\left(e^{-1}\right)\right)\right)=j_{c}^{-1}\left(e \Phi^{-1}\left(s_{\rtimes}\left(\rho\left(e^{-1}\right)\right)\right)\right) .
$$

A straightforward computation now shows that, for $h:=\left(p_{1}^{r} \circ \Phi\right)(e)$,

$$
e \Phi^{-1}\left(s_{\rtimes}\left(\rho\left(e^{-1}\right)\right)\right)=\Phi^{-1}\left(j_{\rtimes}(h)\right)=\Phi^{-1}\left(j_{\rtimes}\left(p_{1}^{r}(\Phi(e))\right)\right) .
$$

Back in (5.21), and noticing that $\Phi \circ j_{K} \circ j_{c}=j_{\rtimes}$, we have

$$
\left(p_{1}^{r} \circ \bar{\Phi}\right)=j_{c}^{-1} \circ \Phi^{-1} \circ j_{\rtimes} \circ\left(p_{1}^{r} \circ \Phi\right)=p_{1}^{r} \circ \Phi .
$$

All together, we conclude that $\bar{\Phi}=\Phi$ and, then, $\widetilde{F}_{R I} \circ \widetilde{F}_{I R}=i d_{\widetilde{\Sigma_{I}}}$, so that $\widetilde{F}_{R I}$ and $\widetilde{F}_{I R}$ are mutually inverse maps.

Last, we specialize Theorem 5.58 to the case of discrete Atiyah sequences.

Corollary 5.59. Let $\pi: Q \rightarrow Q / G$ be a principal $G$-bundle and $\mathcal{U} \subset Q \times Q$ be a symmetric D-type subset. Then, there are bijective correspondences between

(1) $\Sigma_{C}^{e}(\mathcal{U})$, the set of flat discrete connections on $\pi$ with domain $\mathcal{U}$,

(2) $\widetilde{\Sigma_{R}}(\mathcal{U})$, the set of right splittings of the discrete Atiyah sequence over $\mathcal{U}(5.10)$ in the $l L G p d_{Q / G}$ category, and

(3) $\widetilde{\Sigma_{I}}$, the set of isomorphisms from the discrete Atiyah sequence over $\mathcal{U}(5.10)$ to any semidirect product extension of $\mathcal{U}^{\prime \prime}$ by $\widetilde{G}$.

Proof. The equivalence between 1 and 2 is given by Proposition 5.44, while the equivalence between 2 and 3 if given by Theorem 5.58, applied to the extension (5.10)

\section{REFERENCES}

[AHS06] J. Adámek, H. Herrlich, and G. E. Strecker, Abstract and concrete categories: the joy of cats, Repr. Theory Appl. Categ. (2006), no. 17, 1-507, Reprint of the 1990 original [Wiley, New York; MR1051419]. MR 2240597

[And04] I. Androulidakis, Classification of extensions of principal bundles and transitive Lie groupoids with prescribed kernel and cokernel, J. Math. Phys. 45 (2004), no. 10, 3995-4012. MR 2095684

[Ati57] M. F. Atiyah, Complex analytic connections in fibre bundles, Trans. Amer. Math. Soc. 85 (1957), 181-207. MR 0086359

$\left[\mathrm{CdLM}^{+} 06\right]$ J. Cortés, M. de León, J. C. Marrero, D. Martín de Diego, and E. Martínez, A survey of Lagrangian mechanics and control on Lie algebroids and groupoids, Int. J. Geom. Methods Mod. Phys. 3 (2006), no. 3, 509-558. MR 2232870

[CDW87] A. Coste, P. Dazord, and A. Weinstein, Groupoïdes symplectiques, Publications du Département de Mathématiques. Nouvelle Série. A, Vol. 2, Publ. Dép. Math. Nouvelle Sér. A, vol. 87, Univ. Claude-Bernard, Lyon, 1987, pp. i-ii, 1-62. MR 996653

[CR18] A. Casimiro and C. Rodrigo, Reduction of forward difference operators in principal G-bundles, Stat. Optim. Inf. Comput. 6 (2018), no. 1, 42-85. MR 3771534

[DV80] M. Daniel and C.-M. Viallet, The geometrical setting of gauge theories of the YangMills type, Rev. Modern Phys. 52 (1980), no. 1, 175-197. MR 553409

[FTZ10] J. Fernández, C. Tori, and M. Zuccalli, Lagrangian reduction of nonholonomic discrete mechanical systems, J. Geom. Mech. 2 (2010), no. 1, 69-111, MR 2646536

[FTZ16] Lagrangian reduction of discrete mechanical systems by stages, J. Geom. Mech. 8 (2016), no. 1, 35-70, MR 3485921

[FZ] J. Fernández and M. Zuccalli, Discrete connections on principal bundles: holonomy and curvature, in preparation.

[FZ13] A geometric approach to discrete connections on principal bundles, J. Geom. Mech. 5 (2013), no. 4, 433-444, Also, arXiv:1311.0260 [math.DG]. MR 3180706

[GKP11] J. Grabowski, A. Kotov, and N. Poncin, Geometric structures encoded in the Lie structure of an Atiyah algebroid, Transform. Groups 16 (2011), no. 1, 137-160. MR 2785498 
[GP74] V. Guillemin and A. Pollack, Differential topology, Prentice-Hall Inc., Englewood Cliffs, N.J., 1974. MR MR0348781 (50 \#1276)

[Hir76] M. W. Hirsch, Differential topology, Springer-Verlag, New York, 1976, Graduate Texts in Mathematics, No. 33. MR 0448362 (56 \#6669)

[KN96] S. Kobayashi and K. Nomizu, Foundations of differential geometry. Vol. I, Wiley Classics Library, John Wiley \& Sons Inc., New York, 1996, Reprint of the 1963 original, A Wiley-Interscience Publication. MR MR1393940 (97c:53001a)

[Leo04] M. Leok, Foundations of computational geometric mechanics, Ph.D. thesis, California Institue of Technology, 2004.

[LMW05] M. Leok, J. E. Marsden, and A. Weinstein, A discrete theory of connections on principal bundles, arXiv:math/0508338, 2005.

[Mac87] K. Mackenzie, Lie groupoids and Lie algebroids in differential geometry, London Mathematical Society Lecture Note Series, vol. 124, Cambridge University Press, Cambridge, 1987. MR 896907

[Mac05] General theory of Lie groupoids and Lie algebroids, London Mathematical Society Lecture Note Series, vol. 213, Cambridge University Press, Cambridge, 2005. MR 2157566

[MM10] G. Metere and A. Montoli, Semidirect products of internal groupoids, J. Pure Appl. Algebra 214 (2010), no. 10, 1854-1861. MR 2608113

[MMdDM06] J. C. Marrero, D. Martín de Diego, and E. Martínez, Discrete Lagrangian and Hamiltonian mechanics on Lie groupoids, Nonlinearity 19 (2006), no. 6, 1313-1348. MR 2230001 (2007c:37068)

[MMdDM15] _ The local description of discrete mechanics, Geometry, mechanics, and dynamics, Fields Inst. Commun., vol. 73, Springer, New York, 2015, pp. 285-317. MR 3380059

[MMdDS15] J. C. Marrero, D. Martín de Diego, and A. Stern, Symplectic groupoids and discrete constrained Lagrangian mechanics, Discrete Contin. Dyn. Syst. 35 (2015), no. 1, 367-397. MR 3286963

[MW01] J. E. Marsden and M. West, Discrete mechanics and variational integrators, Acta Numer. 10 (2001), 357-514. MR MR2009697 (2004h:37130)

[Pal70] R. S. Palais, When proper maps are closed, Proc. Amer. Math. Soc. 24 (1970), 835-836. MR 0254818

[Wei96] A. Weinstein, Lagrangian mechanics and groupoids, Mechanics day (Waterloo, ON, 1992), Fields Inst. Commun., vol. 7, Amer. Math. Soc., Providence, RI, 1996, pp. 207-231. MR 1365779

Instituto Balseiro, Universidad Nacional de Cuyo - C.N.E.A., Av. Bustillo 9500, Bariloche, RÃo Negro, 8400, República Argentina

Email address: jfernand@ib.edu.ar

Departamento de Matemática, Facultad de Ciencias Exactas, Universidad Nacional de la Plata, Centro de Matemática de La Plata (CMalP), 50 y 115, La Plata, Buenos Aires, 1900, República Argentina

Email address: marianaevaj@gmail.com

Departamento de Matemática, Facultad de Ciencias Exactas, Universidad Nacional de la Plata, Centro de Matemática de la Plata (CMalP), 50 y 115, La Plata, Buenos Aires, 1900, República Argentina

Email address: marce@mate.unlp.edu.ar 